\title{
Spin-locality of higher-spin theories and star-product functional classes
}

\author{
O.A. Gelfond ${ }^{a, b}$ and M.A. Vasiliev ${ }^{a, c}$ \\ ${ }^{a}$ I.E. Tamm Department of Theoretical Physics, Lebedev Physical Institute, \\ Leninsky prospect 53, 119991, Moscow, Russia \\ ${ }^{b}$ Federal State Institution \\ "Scientific Research Institute of System Analysis of Russian Academy of Science", \\ Nakhimovsky prospect 36-1, 117218, Moscow, Russia \\ ${ }^{c}$ Moscow Institute of Physics and Technology, \\ Institutsky pereulok 9, 141701, Dolgoprudny, Moscow region, Russia \\ E-mail: gel@lpi.ru, vasiliev@lpi.ru
}

ABSTRACT: The analysis of spin-locality of higher-spin gauge theory is formulated in terms of star-product functional classes appropriate for the $\beta \rightarrow-\infty$ limiting shifted homotopy proposed recently in [1] where all $\omega^{2} C^{2}$ higher-spin vertices were shown to be spin-local. For the $\beta \rightarrow-\infty$ limiting shifted contracting homotopy we identify the class of functions $\mathcal{H}^{+0}$, that do not contribute to the r.h.s. of HS field equations at a given order. A number of theorems and relations that organize analysis of the higher-spin equations are derived including extension of the Pfaffian Locality Theorem of [2] to the $\beta$-shifted contracting homotopy and the relation underlying locality of the $\omega^{2} C^{2}$ sector of higher-spin equations.

Space-time interpretation of spin-locality of theories involving infinite towers of fields is proposed as the property that the theory is space-time local in terms of original constituent fields $\phi$ and their local currents $J(\phi)$ of all ranks. Spin-locality is argued to be a proper substitute of locality for theories with finite sets of fields for which the two concepts are equivalent.

KeYwORDS: Higher Spin Gravity, Higher Spin Symmetry

ARXIV EPRINT: 1910.00487 


\section{Contents}

1 Introduction $\quad 1$

2 Free fields 3

3 Spin-locality and ultra-locality 5

3.1 Preliminaries 5

$\begin{array}{ll}3.2 & \text { Unfolded equations }\end{array}$

$\begin{array}{lll}3.3 & \text { Spin-locality } & 7\end{array}$

3.4 Ultra-locality 8

$\begin{array}{ll}3.5 & \text { Space-time interpretation }\end{array}$

$\begin{array}{lll}3.6 & \text { Spinor space } & 11\end{array}$

4 Nonlinear higher-spin equations $\quad 12$

5 Perturbative analysis $\quad 14$

$\begin{array}{lll}6 & \text { Star-product functions } & \mathbf{1 5}\end{array}$

$\begin{array}{lll}6.1 & \text { Higher-spin algebra } \mathcal{H} & 15\end{array}$

$\begin{array}{lll}\text { 6.1.1 Star product } & 15\end{array}$

$\begin{array}{lll}6.1 .2 & \text { Inequalities } & 17\end{array}$

$\begin{array}{lll}\text { 6.1.3 Class } \mathcal{H} & 17\end{array}$

$\begin{array}{lll}6.1 .4 & \text { Ideal } \mathcal{I} & 18\end{array}$

$\begin{array}{lll}\text { 6.1.5 } \mathcal{H}^{0+} \text { and } \mathcal{H}^{+0} & 18\end{array}$

$\begin{array}{lll}6.2 & \text { Invariant operations } & 19\end{array}$

$\begin{array}{lll}6.2 .1 & \gamma \text { maps } & 19\end{array}$

$\begin{array}{lll}6.2 .2 & \text { Integration by parts } & 20\end{array}$

$\begin{array}{lll}7 & \text { Shifted homotopy } & 21\end{array}$

7.1 General setup 21

$\begin{array}{ll}7.2 & \text { Shifted homotopy } \\ 7.3 & 22\end{array}$

$\begin{array}{lll}7.3 & \text { Pfaffian Locality Theorem } 25\end{array}$

8 Limiting contracting homotopy and Factorization Lemma 27

$\begin{array}{ll}8.1 & \text { Limiting contracting homotopy } \\ & 27\end{array}$

8.2 Factorization Lemma and limiting cohomology projector 29

$\begin{array}{lll}\text { 8.2.1 Factorization Lemma } & 29\end{array}$

$\begin{array}{ll}\text { 8.2.2 Limiting cohomology projector } & 29\end{array}$

9 Specific form degree relations $\quad 30$

$\begin{array}{lll}9.1 & \text { Star products } & 30\end{array}$

$\begin{array}{lll}9.1 .1 & \mathcal{H}_{0} * \mathcal{H}_{0} & 30\end{array}$

$\begin{array}{lll}\text { 9.1.2 } & \mathcal{H}_{0} * \mathcal{H}_{1} \text { and } \mathcal{H}_{1} * \mathcal{H}_{0} & 32\end{array}$

$\begin{array}{lll}9.1 .3 & \mathcal{H}_{1} * \mathcal{H}_{1} & 33\end{array}$ 
9.2 Limiting contracting homotopy 33

9.2.1 Contracting homotopy of $\mathcal{H}_{1}^{\nu \mu} \quad 34$

$\begin{array}{lll}9.2 .2 & \text { Space } \widetilde{\mathcal{H}}_{1}^{0+} & 34\end{array}$

$\begin{array}{ll}\text { 9.2.3 Contracting homotopy of } \mathcal{H}_{2}^{\nu \mu} & 36\end{array}$

$\begin{array}{ll}10 & \text { Pre-ultra-locality and ultra-locality }\end{array}$

$\begin{array}{ll}10.1 \text { Pre-ultra-locality } & 39\end{array}$

$\begin{array}{lll}\text { 10.1.1 Pre-ultra-local spaces } & 39\end{array}$

$\begin{array}{ll}10.1 .2 \text { Consequences } & 40\end{array}$

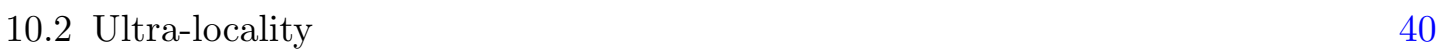

11 Structure relation $\quad 41$

11.1 Summary 41

$\begin{array}{ll}11.2 \text { The proof } & 42\end{array}$

12 Example: ultra-locality of holomorphic $\Upsilon_{2}(\omega, \omega, C, C) \quad 45$

13 Conclusion $\quad 46$

$\begin{array}{ll}\text { A Useful formulae } & 48\end{array}$

$\begin{array}{ll}\text { B Contracting homotopy derivation } & 49\end{array}$

\section{Introduction}

The most symmetric vacuum solution to nonlinear field equations for $4 d$ massless fields of all spins of [3,4] describes $A d S_{4}$. Due to the presence of dimensionful $A d S_{4}$ radius, higher-spin (HS) interactions can contain infinite tails of higher-derivative terms. This can make the theory nonlocal in the standard sense, raising the question which field variables lead to the local or minimally non-local setup in the perturbative analysis as was originally discussed in [5]. In [6-8] it was shown how nonlinear HS equations of [4] reproduce local current interactions in the lowest order in interactions. More recently, in [2, 9] these results were reproduced and extended to some higher-order vertices by an appropriate modification of the conventional homotopy technics of [4].

However, it was not clear how the homotopy technics should be further modified to lead directly to the proper local results in the higher orders of the perturbative analysis of HS equations until recently a new type of limiting shifted homotopy was introduced in [1] allowing to extend the results of the previous work to the vertices up to the fifth order (in the action counting) in the sector of equations on the one-form gauge HS fields. The resulting vertices were shown to be spin-local which means that, as explained in $[1,2,9]$ and in this paper, a vertex is local in the spinor space for any given set of spins. Moreover, as argued below, spin-locality implies usual space-time locality in terms of combinations of 
field variables (like different currents for instance) associated with the primary fields both from the boundary and from the bulk perspective.

The new class of homotopy operators exhibits remarkable properties, partially studied in [1]. The aim of this paper is to extend this analysis using the language of classes of functions developed in [10]. This will allow us to greatly simplify the formalism factoring out the structures that do not contribute to the final result. In this setup, HS equations of [4] provide an extremely powerful tool for the analysis of HS gauge theories directly in the bulk with no reference to $A d S / C F T$ allowing a systematic computation of higher-order HS vertices. We prove useful lemmas that simplify the analysis of the HS equations in general and derive an important relation underlying locality of $\omega^{2} C^{2}$ vertices computed in [1].

There are many reasons why it is important to elaborate the intrinsic analysis of the HS gauge theory in the bulk with no reference to the holographic duals. The simplest is that apart from free boundary theories dual to particular HS gauge theories in the bulk $[11,12]$, the latter equally well describe CFT dual interacting Chern-Simons boundary theories $[13,14]$ where the computation of amplitudes is more involved. More general background solutions of HS theories with more complicated boundary duals like for instance massive deformations can also be of interest.

The approach proposed in this paper is applicable with slight modifications to HS theories in $d=3$ [5] and any $d[15]$ as well as to a more general class of Coxeter HS theories [16] some of which were conjectured to be related to String Theory upon spontaneous breakdown of HS symmetries. Another class of problems where it can be useful includes exact solutions in HS theory like the HS black hole solutions of [17-19]. The results of [1] and of this paper demonstrate great efficiency of the limiting homotopy approach to the analysis of equations of [4]. We are not aware of any other means that could provide a comparably efficient computational scheme in HS gauge theory.

Let us now explain the organization of the rest of the paper highlighting the main results.

We start by recalling by now standard material on free HS fields in section 2 . The concepts of spin-locality, ultra-locality and their space-time interpretation are discussed in section 3. Nonlinear HS equations and general features of their perturbative analysis are recalled in sections 4 and 5 , respectively.

The construction of the class of star-product functions $\mathcal{H}$ introduced in [10] is recalled in section 6 where it is further extended in a way appropriate for the limiting shifted homotopy approach. In particular, $\mathcal{H}$ is represented as a span of two subspaces $\mathcal{H}^{0+}$ and $\mathcal{H}^{+0}$ such that, by Factorization Lemma (8.9), elements of $\mathcal{H}^{+0}$ do not contribute to the dynamical equations at a given order in the limiting homotopy formalism. Also, we identify the two-sided ideal $\mathcal{I}=\mathcal{H}^{0+} \cap \mathcal{H}^{+0}$ elements of which can be discarded in all expressions containing HS gauge fields $\omega$ as not contributing to dynamical field equations within the $\beta \rightarrow-\infty$ limiting homotopy procedure.

The shifted homotopy formalism is recalled in section 7. Namely, after recalling the general setup in section 7.1, expressions for contracting homotopy and cohomology projector are presented in section 7.2 for a general $\beta$-shift. In section 7.3, Pfaffian Locality Theorem (PLT) of [2] is extended to the $\beta$-dependent contracting homotopies. This will 
be used later in section 9.2.3 as the instrumental tool for the proof of ultra-locality of the vertices in question.

The limit $\beta \rightarrow-\infty$ is considered in section 8 . Namely, in section 8.1 we derive the limiting contracting homotopy formulae which underly the Pre-Ultra-Locality Theorem of section 9.2.3. In section 8.2 the formula for limiting cohomology projector is derived from which simple but important Factorization Lemma follows showing that elements of $\mathcal{H}^{+0}$ do not contribute to field equations on physical fields.

A priori, application of the limiting homotopy prescription to general elements of $\mathcal{H}$ may not be well defined leading to the one-forms $W$ divergent in the $\beta \rightarrow-\infty$ limit. This would not imply any divergency in the HS equations, the perturbative analysis of which is well defined for any finite $\beta<1$, but rather the inapplicability of the limiting homotopy indicating potential non-locality of the theory. Hence, for the analysis of locality, it is important to have a sufficient criterion guaranteeing that this does not happen. Details of this analysis depend on a degree of the differential forms in the anticommuting spinorial differentials $\theta^{\alpha}$.

Specificities of the spaces $\mathcal{H}_{p}$ of $p$-forms in $\theta$ are studied in section 9. In section 9.1 we collect useful formulae on star products of elements of various spaces $\mathcal{H}_{p}$ up to terms in the ideal $\mathcal{I}$. Then in section 9.2.1 we analyse properties of the limiting homotopy applied to $\mathcal{H}_{1}$. It is shown that, generally, application of the limiting homotopy to $\mathcal{H}_{1}^{0+}$ can lead to infinity and a subspace $\widetilde{\mathcal{H}}_{1} \subset \mathcal{H}_{1}$ is identified in section 9.2 .2 , such that application of the limiting homotopy to $\widetilde{\mathcal{H}}_{1}$ has a well defined limit $\beta \rightarrow-\infty$. In turn, in section 9.2.3 it is shown that $\triangle_{q,-\infty} \mathcal{H}_{2}^{+0} \subset \widetilde{\mathcal{H}}_{1}$. It is also shown here that the $y$-dependent part of $\triangle_{q,-\infty} \mathcal{H}_{2}^{+0}$ belongs to $\mathcal{H}_{1}^{+0}$ which does not contribute to the field equations by Factorization Lemma.

In section 10, we introduce the notion of pre-ultra-locality, underlying the analysis of spin-locality of HS equations. It is shown here that the contribution resulting from $\triangle_{q,-\infty} \mathcal{H}_{2}^{+0}$ not only is well defined in the limit $\beta \rightarrow-\infty$ but is also pre-ultra-local that, in accordance with PLT, guarantees ultra-locality in the second order in HS zero-forms $C$.

In section 11 we prove the relation underlying analysis of HS vertices in the second order in zero-forms $C$. Structure Relation considered in section 11 proves that r.h.s. of the second-order part of nonlinear HS equations is indeed in $\mathcal{H}_{2}^{+0}$ meeting the conditions of Pre-Ultra-Locality Theorem. This implies that the part of the vertex bilinear in the zero-forms $C$ is ultra-local.

Finally in section 12 the efficiency of the developed methods is illustrated by an elementary computation-free proof of spin-locality of the holomorphic part of the vertex $\Upsilon_{2}(\omega, \omega, C, C)$ evaluated in [1].

Conclusions are in section 13. Some useful formulae are collected in appendix A. Details of the derivation of the $\beta$-dependent shifted contracting homotopy are given in appendix B.

\section{Free fields}

The formulation of [4] uses the language of spinors. Its relation to the conventional setup in terms of space-time derivatives is via unfolded equations as we briefly recall now. 
Unfolded equations of $4 d$ massless Fronsdal [20-23] fields of all spins $s=$ $0,1 / 2,1,3 / 2,2 \ldots$ in $A d S_{4}$ are formulated in terms of a one-form $\omega(Y ; K \mid x)=$ $d x^{n} \omega_{n}(Y ; K \mid x)$ and zero-form $C(Y ; K \mid x)[24], Y^{A}=\left(y^{\alpha}, \bar{y}^{\dot{\alpha}}\right) .{ }^{1}$ Klein operators $K=(k, \bar{k})$ satisfy

$$
k y^{\alpha}=-y^{\alpha} k, \quad k \bar{y}^{\dot{\alpha}}=\bar{y}^{\dot{\alpha}} k, \quad \bar{k} y^{\alpha}=y^{\alpha} \bar{k}, \quad \bar{k} \bar{y}^{\dot{\alpha}}=-\bar{y}^{\dot{\alpha}} \bar{k}, \quad k k=\bar{k} \bar{k}=1, \quad k \bar{k}=\bar{k} k .
$$

To describe massless fields, the one-form $\omega(Y ; K \mid x)$ and zero-form $C(Y ; K \mid x)$ should be, respectively, even and odd in $k, \bar{k}$. As a result, massless fields are doubled

$$
C(Y ; K \mid x)=C^{1,0}(Y \mid x) k+C^{0,1}(Y \mid x) \bar{k}, \quad \omega(Y ; K \mid x)=\omega^{0,0}(Y \mid x)+\omega^{1,1}(Y \mid x) k \bar{k} .
$$

Unfolded field equations for free massless fields of all spins in $A d S_{4}$ are [24]

$$
\begin{aligned}
R_{1}(Y ; K \mid x) & =\frac{i}{4}\left(\eta \bar{H}^{\dot{\alpha} \dot{\beta}} \frac{\partial^{2}}{\partial \bar{y}^{\dot{\alpha}} \partial \bar{y}^{\dot{\beta}}} C(0, \bar{y} ; K \mid x) k+\bar{\eta} H^{\alpha \beta} \frac{\partial^{2}}{\partial y^{\alpha} \partial y^{\beta}} C(y, 0 ; K \mid x) \bar{k}\right), \\
\tilde{D} C(Y ; K \mid x) & =0
\end{aligned}
$$

where $\eta$ is a free phase parameter and

$$
\begin{aligned}
R_{1}(Y ; K \mid x) & :=D^{a d} \omega(Y ; K \mid x):=D^{L} \omega(Y ; K \mid x)+\lambda h^{\alpha \dot{\beta}}\left(y_{\alpha} \frac{\partial}{\partial \bar{y}^{\dot{\beta}}}+\frac{\partial}{\partial y^{\alpha}} \bar{y}_{\dot{\beta}}\right) \omega(Y ; K \mid x), \\
\tilde{D} C(Y ; K \mid x) & :=D^{L} C(Y ; K \mid x)-i \lambda h^{\alpha \dot{\beta}}\left(y_{\alpha} \bar{y}_{\dot{\beta}}-\frac{\partial^{2}}{\partial y^{\alpha} \partial \bar{y}^{\dot{\beta}}}\right) C(Y ; K \mid x), \\
D^{L} f(Y ; K \mid x) & :=\mathrm{d}_{x} f(Y ; K \mid x)+\left(\omega_{L}^{\alpha \beta} y_{\alpha} \frac{\partial}{\partial y^{\beta}}+\bar{\omega}_{L}^{\dot{\alpha} \dot{\beta}} \bar{y}_{\dot{\alpha}} \frac{\partial}{\partial \bar{y}^{\dot{\beta}}}\right) f(Y ; K \mid x), \quad \mathrm{d}_{x}:=d x^{n} \frac{\partial}{\partial x^{n}} .
\end{aligned}
$$

Background $A d S_{4}$ space of radius $\lambda^{-1}=\rho$ is described by a flat $s p(4)$ connection

$$
\omega_{0}=\left(w_{\alpha \beta}, \bar{w}_{\dot{\alpha} \dot{\beta}}, h_{\alpha \dot{\beta}}\right)
$$

containing Lorentz connection $w_{\alpha \beta}, \bar{w}_{\dot{\alpha} \dot{\beta}}$ and vierbein $h_{\alpha \dot{\beta}}$, that obey

$$
\begin{aligned}
& \mathrm{d}_{x} w_{\alpha \beta}+w_{\alpha \gamma} w_{\beta}^{\gamma}-\lambda^{2} H_{\alpha \beta}=0, \\
& \mathrm{~d}_{x} \bar{w}_{\dot{\alpha} \dot{\beta}}+\bar{w}_{\dot{\alpha} \dot{\gamma}} \bar{w}_{\dot{\beta}}^{\dot{\gamma}}-\lambda^{2} \bar{H}_{\dot{\alpha} \dot{\beta}}=0, \\
& \mathrm{~d}_{x} h_{\alpha \dot{\beta}}+w_{\alpha \gamma} h_{\dot{\beta}}^{\gamma}+\bar{w}_{\dot{\beta} \dot{\delta}} h_{\alpha}{ }^{\dot{\delta}}=0 .
\end{aligned}
$$

Here $H^{\alpha \beta}:=h^{\alpha \dot{\alpha}} h_{\dot{\alpha}}^{\beta}$ and $\bar{H}^{\dot{\alpha} \dot{\beta}}:=h^{\alpha \dot{\alpha}} h_{\alpha}^{\dot{\beta}}$ are the frame two-forms (wedge symbol is omitted).

In the massless sector, system (2.3), (2.4) decomposes into subsystems of different spins, with a spin $s$ described by the one-forms $\omega(y, \bar{y} ; K \mid x)$ and zero-forms $C(y, \bar{y} ; K \mid x)$ obeying

$$
\omega(\mu y, \mu \bar{y} ; K \mid x)=\mu^{2(s-1)} \omega(y, \bar{y} ; K \mid x), \quad C\left(\mu y, \mu^{-1} \bar{y} ; K \mid x\right)=\mu^{ \pm 2 s} C(y, \bar{y} ; K \mid x),
$$

\footnotetext{
${ }^{1} A=1, \ldots 4$ is a Majorana spinor index while $\alpha=1,2$ and $\dot{\alpha}=1,2$ are two-component ones raised and lowered by $\varepsilon_{\alpha \beta}=-\varepsilon_{\beta \alpha}, \varepsilon_{12}=1: A^{\alpha}=\varepsilon^{\alpha \beta} A_{\beta}, A_{\alpha}=A^{\beta} \varepsilon_{\beta \alpha}$ and analogously for dotted indices.
} 
where + and - correspond to helicity $h= \pm s$ selfdual and anti-selfdual parts of the generalized Weyl tensors $C(y, \bar{y} ; K \mid x)$. For spins $s \geq 1$, equation (2.3) expresses the Weyl zero-forms $C(Y ; K \mid x)$ via gauge invariant combinations of derivatives of the HS gauge connections. The primary-like Weyl zero-forms are just the holomorphic and antiholomorphic parts $C(y, 0 ; K \mid x)$ and $C(0, \bar{y} ; K \mid x)$ which appear on the r.h.s. of eq. (2.3). Those associated with higher powers of auxiliary variables $y$ and $\bar{y}$ describe on-shell nontrivial combinations of derivatives of the generalized Weyl tensors as is obvious from eqs. (2.4), (2.6) relating second derivatives in $y, \bar{y}$ to the $x$ derivatives of $C(Y ; K \mid x)$ of lower degrees in $Y$. Hence, higher derivatives in the nonlinear system hide in the components of $C(Y ; K \mid x)$ of higher orders in $Y$. To see whether the resulting equations are local or not at higher orders one has to inspect the dependence of vertices on the higher components of $C(Y ; K \mid x)$.

At the linearized level, eq. (2.6) implies that $\frac{\partial}{\partial x}$ is equivalent to $\frac{\partial^{2}}{\partial y \partial \bar{y}}$. Hence, at this level the analysis of spin-locality in terms of $y, \bar{y}$ variables is equivalent to that in terms of space-time derivatives. However in higher orders eq. (2.6) acquires nonlinear corrections making the relation between the two formalisms less straightforward but still tractable as we explain now.

\section{Spin-locality and ultra-locality}

\subsection{Preliminaries}

HS gauge theories have two main features distinguishing them from usual local field theories.

The first one is that they demand non-zero background curvature which implies $(A) d S$ background geometry of non-zero radius $\rho=\lambda^{-1}$ in the most symmetric case. This allows HS gauge theories to contain higher derivatives at the interaction level, that enter in the dimensionless combination $\rho D_{n}$, where $D_{n}$ is the Lorentz covariant background derivative. This has two important consequences. First is that the dimensionless combination $\rho D_{n}$ can enter HS interactions in any degree. Second is that, in the absence of other dimensionful parameters, which is the case in the HS theory with unbroken HS symmetries, one cannot apply the low-energy expansion neglecting higher-derivative terms because the dimensionless combinations of covariant derivatives $\rho D_{n}$ are of order one since their commutator is

$$
\left[\rho D_{n}, \rho D_{m}\right]=\rho^{2} R_{n m} \sim(\rho \lambda)^{2} \sim 1
$$

Hence whether a theory is local or not is characterized by the behaviour of the coefficients $a_{k}(p)$ in the power-series expansions of the order- $k$ vertex

$$
\sum_{p_{i}} a_{k}(p)(\rho D)^{p_{1}} \phi_{1}(x) \ldots(\rho D)^{p_{k}} \phi_{k}(x)
$$

with various elementary fields $\phi_{i}$, i.e. spin $s_{i}$ Fronsdal fields in HS gauge theory $\left(p_{i}\right.$ are degrees of the derivatives while Lorentz indices are implicit). A theory with finite number of fields can be called local if any order- $k$ vertex contains at most a finite number of non-zero coefficients $a_{k}\left(p_{i}\right)$. 
Another fundamental feature of HS gauge theories is that they contain infinite towers of fields of arbitrarily high spins. This means that the theory contains an infinite number of vertices at any given order simply because index $i$ of $\phi_{i}$ takes an infinite number of values. Apart from its order, the vertex is characterised by the pattern of fields entering. Assuming that different fields are fully characterized by their spins $\left\{s_{i}\right\}$ (or generalized spins if there are some other quantum numbers), different vertices are characterised by different sets of (generalized) spins. Naively, a proper substitute of the usual concept of locality is the condition that each vertex of particular order $k$ and spin pattern $\left\{s_{i}\right\}$ is local. This is known to be the case in HS theory at the lowest (cubic) order since [25-30] giving a simplest example of spin-locality in the models with infinite towers of massless fields. The higher-order extension of the notion of spin-locality proposed in this paper is slightly more sophisticated but the same in spirit. This is most naturally formulated in terms of unfolded dynamics as we explain now.

\subsection{Unfolded equations}

Nonlinear corrections to unfolded equations on physical fields $\omega(Y)$ and $C(Y)$ extending Central-on-shell theorem (2.3), (2.4) to higher orders can be packed into the form

$$
\begin{aligned}
\mathrm{d}_{x} \omega & =-\omega * \omega+\Upsilon_{1}^{\omega}\left(\omega^{2}, C\right)+\Upsilon_{2}^{\omega}\left(\omega^{2}, C^{2}\right)+\ldots+\Upsilon_{n}^{\omega}\left(\omega^{2}, C^{n}\right)+\ldots, \\
\mathrm{d}_{x} C & =-[\omega, C]_{*}+\Upsilon_{2}^{C}\left(\omega, C^{2}\right)+\Upsilon_{3}^{C}\left(\omega, C^{3}\right)+\ldots+\Upsilon_{n}^{C}\left(\omega, C^{n}\right)+\ldots,
\end{aligned}
$$

where $*$ is the Moyal star product acting on the commuting spinor variables $Y$ in $\omega(Y ; K \mid x)$ and $C(Y ; K \mid x)$

$$
(f * g)(Y)=\int \frac{d^{4} U d^{4} V}{(2 \pi)^{4}} \exp \left[i U^{A} V^{B} C_{A B}\right] f(Y+U) g(Y+V),
$$

$C_{A B}=\left(\epsilon_{\alpha \beta}, \bar{\epsilon}_{\dot{\alpha} \dot{\beta}}\right)$ is the $4 d$ charge conjugation matrix and $U^{A}, V^{B}$ are real integration variables.

Generally, (for more detail see, e.g., [32] and references therein) any dynamical system can be described by unfolded equations of the form

$$
\mathrm{d}_{x} W^{\Omega}=G^{\Omega}(W),
$$

where $W^{\Omega}$ is some set of differential forms and $G^{\Omega}(W)$ obeys the compatibility condition

$$
G^{\Phi}(W) \frac{\partial G^{\Lambda}}{\partial W^{\Phi}}=0
$$

In the HS theory in question the set of differential forms $W^{\Omega}$ consists of (components of the expansion in powers of $Y$ of $)$ the one-form $\omega(Y ; K \mid x)$ and zero-form $C(Y ; K \mid x)$. In this setup space-time is described by a vacuum one-form $\omega_{0}(2.8)$ that obeys the flatness condition

$$
\mathrm{d}_{x} \omega_{0}+\omega_{0} * \omega_{0}=0
$$


for a Lie algebra $h$ describing symmetry of space-time. In the $4 d$ HS theory, vacuum symmetry is $s p(4)$ with $A d S_{4}$ as associated space-time. The flatness condition is (2.9). Thus in (3.3), (3.4) one has

$$
\omega=\omega_{0}+\omega^{\prime}
$$

where $\omega^{\prime}(Y \mid x)$ is a perturbative fluctuation.

At the linearized level unfolded equations have a form of covariant constancy conditions on the first-order fields valued in some $h$-modules. For instance, zero-forms $C(Y, K \mid x)$ describe a sum of $s p(4)$-modules $V_{s}$ associated with massless fields of all spins $s$ via (2.10). The covariant constancy conditions are (2.4). These modules contain primary-like fields which in the HS case are the generalized Weyl-tensor like components $C(0, \bar{y} ; K \mid x)$ and $C(y, 0 ; K \mid x)$ that appear on the r.h.s. of (2.3) and their descendants $C(y, \bar{y} ; K \mid x)$ depending both on $y$ and on $\bar{y}$. Primary-like fields are those that belong to the $\sigma_{-}$-cohomology [31] (for more detail see [32] and references therein.) For instance, in the sector of HS zero-forms $C(y, \bar{y} \mid x)$

$$
\sigma_{-}=i \lambda h^{\alpha \dot{\beta}} \frac{\partial^{2}}{\partial y^{\alpha} \partial \bar{y}^{\dot{\beta}}}, \quad \sigma_{-}^{2}=0 .
$$

Descendants are expressed via space-time derivatives of the primaries as follows in particular from (2.4), (2.6). In fact, for $s \geq 1$ true HS primary fields are Fronsdal fields that belong to $\sigma_{-}$-cohomology in the one-form sector of $\omega(Y \mid x)$. To simplify notation in the general consideration of this section we will denote primary-like Fronsdal components $\phi$ and their descendants in $\omega^{\prime}(3.9)$ and $C$ as $\mathcal{C}$.

\subsection{Spin-locality}

Nonlinear corrections bring to r.h.s. of unfolded equations multilinear products of the original constituent fields $\mathcal{C}$. These are valued in the tensor products $V_{s_{1}} \otimes \ldots \otimes V_{s_{k}}$. As a result, multilinear products of the constituent fields describe higher $h$-modules $V_{s_{1} \ldots s_{k}}^{\mathbf{t}}$, where $\mathbf{t}$ is a multiindex distinguishing between different irreducible components in $V_{s_{1}} \otimes$ $\ldots \otimes V_{s_{k}}$. For instance, in the bilinear case, $V_{s_{1} s_{2}}^{t}$ forms the spin- $t$ current module built from the spin- $s_{1}$ and $s_{2}$ constituent modules. In other words, $V_{s_{1} s_{2}}^{t}$-valued fields $\mathcal{J}_{s_{1} s_{2}}^{t}$ are spin- $t$ currents, built from the constituent fields of spins $s_{1}$ and $s_{2}$, along with all their descendants. These obey their own rank-two unfolded equations [33] expressing the current conservation conditions. Usual conserved currents are the primary-like components $J_{s_{1} s_{2}}^{t}(\mathcal{C}, \mathcal{C})$ of the current system $\mathcal{J}_{s_{1} s_{2}}^{t}(\mathcal{C}, \mathcal{C})$, with respect to the rank-two $\sigma_{-}$-cohomology. As such, $\mathcal{J}_{s_{1} s_{2}}^{t}(\mathcal{C}, \mathcal{C})$ are local combinations of the constituent fields $\mathcal{C}$ which means that the primary currents $J_{s_{1} s_{2}}^{t}(\mathcal{C}, \mathcal{C})$ are expressed via a finite number of descendants among $\mathcal{C}$ for given spins $s_{1}$ and $s_{2}$.

Analogously, higher currents by construction are local with primary components $J_{\vec{s}}^{\mathbf{t}}(\mathcal{C}, \mathcal{C}, \ldots)$ expressed via a finite number of descendants $\mathcal{C}$ of $\phi$ for any fixed set of $\mathbf{t}, \vec{s}=\left(s_{1}, s_{2}, \ldots\right)$ where (generalized) spin $\mathbf{t}$ labels irreducible $h$-modules in the tensor product of spin- $s_{i} h$-modules associated with $\mathcal{C}_{s_{i}}$. This is what normally happens automatically as a consequence of the unfolded machinery. The nontrivial question on the form of unfolded equations is whether the nonlinear corrections to, say, equations (3.3) and (3.4), 
contain a finite number of descendants of higher currents $\mathcal{J}_{\vec{s}}^{\mathbf{t}}(\mathcal{C}, \mathcal{C}, \ldots)$ for any (generalized) spin $\mathbf{t}$ of the fields on l.h.s. or not. If this is the case, we call equations spin-local. The nonlinear corrections due to spin-local vertices are local in the standard space-time sense at least in the perturbative order at which they first appear. If r.h.s. contains infinite chains of descendants, that can happen in presence of the cosmological constant destroying the exact derivative grading typical for conformal field theory, the system is not spin-local. In the latter case we will call such a system spin-nonlocal. Indeed, the resulting vertices are nonlocal already at the order they first appear.

It should be noted that one has to distinguish between essentially spin-nonlocal systems and seemingly spin-nonlocal ones resulting from the application of a nonlocal field redefinition involving infinite tower of descendants to some (may be unknown) spin-local system. In the latter case, the potentially difficult problem is to find an underlying spinlocal system. An example of the unfolded system allowing no spin-local form at all is provided by the HS models of [4] with nonlinear function $F_{*}(B)$ instead of the linear one $\eta B$ as in this paper (see also Conclusion).

Thus, spin-locality of the unfolded equations is achieved once all order- $n$ corrections to unfolded equations $\Upsilon_{n}^{\omega}\left(\omega^{2}, C^{n}\right)$ and $\Upsilon_{n}^{C}\left(\omega, C^{n}\right)$ are spin-local containing a finite number of descendants for any external (generalized) spin $\mathbf{t}$ and spins $s_{i}$ of the constituent fields $\mathcal{C}$. If, on the other hand, the vertices contain infinite towers of descendants of $J_{\vec{s}}^{\mathbf{t}}(\mathcal{C}, \mathcal{C}, \ldots)$ for some $\vec{s}$ and/or $\mathbf{t}$ then they are spin-nonlocal.

Spin-local field redefinitions are defined analogously

$$
\delta J_{\vec{s}}^{\mathbf{t}}=\sum a\left(\mathbf{t}, \vec{s} ; \mathbf{t}^{1}, \vec{s}^{1} ; \mathbf{t}^{2}, \vec{s}^{2} ; \ldots\right) J_{\vec{s}^{1}}^{\mathbf{t}^{1}} \ldots J_{\vec{s}^{k}}^{\mathbf{t}^{k}}
$$

containing at most a finite number of descendants of any multilinear current $J_{\vec{s}^{j}}^{\mathbf{t}^{j}}$. Field redefinitions (3.11) involving infinite number of descendants of $J_{\vec{s}^{j}}^{\mathbf{t}^{j}}$ are genuinely spinnonlocal. Here the variation of higher currents is deduced from that of the lower ones. For instance, the spin-local field redefinition induced by the bilinear current variation

$$
\begin{aligned}
\delta \mathcal{C}_{t} & =a \mathcal{J}_{s_{1} s_{2}}^{t}\left(\mathcal{C}_{s_{1}}, \mathcal{C}_{s_{2}}\right) \\
\delta \mathcal{J}_{s_{1} s_{2}}^{t}\left(\mathcal{C}_{s_{1}}, \mathcal{C}_{s_{2}}\right) & =\mathcal{J}_{s_{1} s_{2}}^{t}\left(\delta \mathcal{C}_{s_{1}}, \mathcal{C}_{s_{2}}\right)+\mathcal{J}_{s_{1} s_{2}}^{t}\left(\mathcal{C}_{s_{1}}, \delta \mathcal{C}_{s_{2}}\right)
\end{aligned}
$$

turns out to be bilinear in the rank-one fields $\mathcal{C}$ and rank-two fields $\mathcal{J}$. Clearly, spin-local field redefinitions form a group: any combination of spin-local transformations gives a new spin-local transformation. The inverse transformation also exists within the perturbative expansion in powers of local currents.

If such a field redefinition involves an infinite number of descendants of some rank- $n$ currents starting from the rank-one case of $\mathcal{C}$, the field redefinition is not spin-local being essentially nonlocal.

\subsection{Ultra-locality}

Spin-local vertices and field redefinitions admit an ultra-local subclasses, in which all vertices in the unfolded equations are spin-local in terms of the original fields $\mathcal{C}_{i}$, i.e. without involvement of higher-rank currents. More precisely, spin-local vertices are called ultra-local 
if all nonlinear corrections to unfolded HS equations through the currents $\mathcal{J}_{\vec{s}}^{\mathbf{t}}(\mathcal{C}, \mathcal{C}, \ldots)$ are such that for any given set of spins $\vec{s}$ of the constituent fields, only currents $\mathcal{J}_{\vec{s}}^{\mathbf{t}}$ with a finite set of generalized spins $\mathbf{t}$ contribute.

The group of ultra-local field redefinitions that leave this class invariant is defined analogously as such field redefinitions (containing a finite number of descendants of the constituent fields)

$$
\delta \mathcal{C}_{t}=\sum a\left(t \mid s_{1}, s_{2}, \ldots\right) \mathcal{C}_{s_{1}} \ldots \mathcal{C}_{s_{k}}
$$

that for any set of spins of the constituent fields $s_{i}$, only a finite number of the coefficients $a\left(t \mid s_{1}, s_{2}, \ldots\right)$ are non-zero at different $t$. Clearly, ultra-local field redefinitions leave the class of ultra-local vertices invariant. Indeed, any term like

$$
\delta \mathcal{J}^{\mathbf{t}}\left(\mathcal{C}_{s_{1}} \ldots, \delta \mathcal{C}_{s_{i}}, \ldots \mathcal{C}_{s_{l}}\right)=\mathcal{J}^{\mathbf{t}}\left(\mathcal{C}_{s_{1}} \ldots, \sum a\left(s_{i} \mid s_{1}^{\prime}, s_{2}^{\prime}, \ldots\right) \mathcal{C}_{s_{1}^{\prime}} \ldots \mathcal{C}_{s_{k}^{\prime}}, \ldots \mathcal{C}_{s_{l}}\right)
$$

is ultra-local provided that both the original current and the transformation (3.14) were. Ultra-locality property of the vertices of the unfolded equations (3.3) implies, as we show now, that space-time equations that follow from (3.3) are space-time local in the usual sense.

\subsection{Space-time interpretation}

Unfolded HS equations acquire nonlinear corrections (3.3), (3.4). In the lowest order, these are interactions with currents which, in the sector of zero-forms, have the structure

$$
\mathrm{d}_{x} C^{s}=-\left.[\omega, C]_{*}\right|^{s}+\sum_{s_{1}, s_{2}} \mathcal{J}_{s_{1} s_{2}}^{s}\left[C_{s_{1}}, C_{s_{2}}\right]+\ldots
$$

where $\mathcal{J}_{s_{1} s_{2}}^{s}\left[C_{s_{1}}, C_{s_{2}}\right]$ denotes the spin- $s$ current along with its descendants bilinear in the massless fields of spins $s_{1}$ and $s_{2}$. These currents are local $[6,9]$. However, their appearance affects the relation between the fields $C$ and space-time derivatives. Extracting the vacuum part (3.16) can schematically be written in the form

$$
\begin{aligned}
D^{L} C^{s}(Y ; K \mid x)= & i \lambda h^{\alpha \dot{\beta}}\left(y_{\alpha} \bar{y}_{\dot{\beta}}-\frac{\partial^{2}}{\partial y^{\alpha} \partial \bar{y}^{\dot{\beta}}}\right) C^{s}(Y ; K \mid x) \\
& +\sum_{s_{1}, s_{2}=0}^{\infty} \mathcal{J}^{s}\left(C_{s_{1}}(Y ; K \mid x), C_{s_{2}}(Y ; K \mid x)\right)+\ldots
\end{aligned}
$$

with $D^{L}(2.7)$.

Eq. (3.17) means that the interpretation of the components of $C$ in terms of spacetime derivatives upon elimination of the $y, \bar{y}$-dependence acquires $\mathcal{J}$-dependent corrections. Derivation of bilinear current contribution to dynamical equations that follows from the nonlinear HS equations has been done in [7] in the lowest order in interactions. Proceeding analogously, one can obtain from (3.17) space-time equations of the structure

$$
L^{\mathrm{FR}} \phi_{s}=\sum_{s_{1}, s_{2}=0}^{\infty} J_{s_{1} s_{2}}^{s}\left[\phi_{s_{1}}, \phi_{s_{2}}\right]+\sum_{t=0}^{\infty} \sum_{s_{1}, s_{2}, s_{3}} J_{s_{1} t}^{s}\left[\phi_{s_{1}}, \mathcal{J}_{s_{2} s_{3}}^{t}\right]+\ldots
$$


where $\phi_{s}$ is a spin- $s$ Fronsdal field, $L^{\mathrm{FR}} \phi_{s}$ is 1.h.s. of free Fronsdal equations and, for a given spin $s$, at most a finite number of components (descendants) of every current contribute.

The class of ultra-local unfolded equations obviously leads to local theories in terms of original constituent fields $\phi_{s}$. Indeed, in this case any sum over $t$ in the vertex (3.18) is finite, hence remaining local for any fixed set of spins of $\phi_{s}$. This makes the class of ultra-local equations most welcome in the analysis of locality of HS theory. Let us stress that in [1] it has been shown that all (anti)holomorphic $C^{2}$ vertices (i.e. those proportional to $\left.\left(\bar{\eta}^{2}\right) \eta^{2}\right)$ are ultra-local. However mixed vertices proportional to $\eta \bar{\eta}$ are spin-local but not ultra-local.

In the spin-local but not ultra-local case, locality is restored once currents in corrections to Fronsdal equations are treated as independent fields. The resulting terms are still local containing a finite number of derivatives of each current. Usual space-time locality of the spin-local vertices is not obvious. Indeed, if expressions for currents $J_{s_{2} s_{3}}^{t}=J_{s_{2} s_{3}}^{t}\left[\phi_{s_{2}}, \phi_{s_{3}}\right]$ in terms of constituent fields are plugged into (3.18), this can lead to expressions with an arbitrary number of derivatives of the constituent fields due to infinite summation over $t$ because the same fields $\phi$ contribute to currents of different spins. For instance, two spin-zero fields generate currents $J_{00}^{t}\left[\phi_{0}, \phi_{0}\right]$ of any spin $t$ where the number of derivatives of $\phi_{0}$ increases with $t$. By this mechanism, formula (3.18) can bring corrections with the infinite number of derivatives of the same constituent fields. Clearly, this phenomenon is specific for theories containing infinite towers of fields allowing an infinite summation over $t$ in (3.18). Thus, spin-locality is equivalent to usual locality for the models with finite number of fields but may not be equivalent for the models with infinite number of fields. Careful analysis of the level of (non)locality of the spin-local HS system needs further system-dependent investigation being beyond the scope of this paper. On the other hand, if r.h.s. of field equations cannot be expressed in terms of local expressions of the constituent fields and associated currents, such a theory should be treated as essentially nonlocal. This can happen, in particular, as a result of application of a nonlocal field redefinition to a spin-local HS theory.

Note that, being associated with $h$-modules, the currents $\mathcal{J}_{\vec{s}}^{\mathbf{t}}(\mathcal{C}, \mathcal{C}, \ldots)$ can be interpreted as operators of the boundary operator algebra within $A d S / C F T$ paradigm with $h$ interpreted as the boundary conformal symmetry. A related point is that higher currents have clear meaning in terms of homological resolution underlying unfolded formulation of HS equations. For instance, spin-local bilinear (rank two) currents $J_{2}(C, C$ ) that appear on r.h.s. of $4 d$ massless field equations are primary fields of a rank-two module of the $4 d$ HS algebra [33]. The latter can themselves be interpreted as $6 d$ massless fields [34]. As such, they admit currents built from $6 d$ massless fields that, in turn, can be interpreted as rank-four currents $J_{4}\left(J_{2}, J_{2}\right)$ from the $4 d$ perspective, having local form in terms of $J_{2}$. This process continues indefinitely [35].

Though corrections (3.18) are seemingly reminiscent of the current-exchange-like contributions resulting from the holographic reconstruction [36], spin-locality implies that unfolded equations acquire only spin-local corrections which may be non-local in terms of constituent fields but have local form in terms of bilinear and higher currents associated with the boundary conformal fields. This form of interactions is essentially different 
from the standard current-exchange corrections being heavily non-local in terms of currentdependent corrections as emphasized e.g. in [36]. Needless to say that in the ultra-local case all these corrections are local in the standard sense.

General field redefinitions that naturally appear in the unfolded field equations are not spin-local, mixing all descendant fields for all spins. Application of such a field redefinition to an ultra-local or spin-local version of the model in question drives it away from the spin-local form. Hence, in the unfolded formalism one of the key questions is to find an appropriate set of variables in which the equations have spin-local form. This is the issue in which the progress was made in $[1,2,6,7,9]$ (and references therein) and in this paper by showing that all $C^{2}$ corrections to HS equations can be brought to spin-local form.

\subsection{Spinor space}

Since, as recalled in the next section (see also [37]) the structure of $4 d$ HS equations is determined by their spinor sector, all concepts in HS theory including locality have to admit proper interpretation in terms of auxiliary spinor variables. Here we explain how spin-locality and ultra-locality emerge in these terms.

As explained in [2], general exponential representation for the order- $n$ corrections in the zero-forms $C$ can be put into the form

$$
\left.\sum_{\mathbf{p} \overline{\mathbf{p}}} \int d \tau \hat{\mathcal{P}}_{n}^{\mathbf{p} \overline{\mathbf{p}}}(y, \bar{y}, p, \bar{p}, \tau) \hat{E}_{n}^{\mathbf{p} \overline{\mathbf{p}}}(y, \bar{y}, p, \bar{p}, \tau) C\left(Y_{1} ; K\right) \ldots C\left(Y_{n} ; K\right)\right|_{Y_{j}=0},
$$

where

$$
p_{\alpha}^{j}:=-i \frac{\partial}{\partial y_{j}^{\alpha}}, \quad \bar{p}_{\dot{\alpha}}^{j}:=-i \frac{\partial}{\partial \bar{y}_{j}^{\dot{\alpha}}},
$$

$\hat{\mathcal{P}}_{n}^{\mathbf{p} \overline{\mathbf{p}}}(y, \bar{y}, p, \bar{p}, \tau)$ is some polynomial of $y, \bar{y}, p^{i}$ and $\bar{p}^{i}$ with coefficients being regular functions of some homotopy integration parameters $\tau$, and

$$
\hat{E}_{n}^{\mathbf{p} \overline{\mathbf{p}}}=\hat{E}_{n}^{\mathbf{p}} \hat{\bar{E}}_{n}^{\overline{\mathbf{p}}}, \quad \hat{E}_{n}^{\mathbf{p}}(\hat{B}, \hat{P}, p \mid y)=\exp i\left(-\hat{B}_{j}(\tau) p_{\alpha}^{j} y^{\alpha}+\frac{1}{2} \hat{P}_{i j}(\tau) p^{i \alpha} p_{\alpha}^{j}\right) k^{\mathbf{p}},
$$

where $\mathbf{p}=0,1$ while $\hat{B}_{j}(\tau)$ and $\hat{P}_{i j}(\tau)=-\hat{P}_{j i}(\tau)$ are some $\tau$-dependent coefficients.

Spin-locality of HS interactions is governed by the coefficients $\hat{P}_{i j}$ in $\hat{E}_{n}^{\mathbf{p}}$ (3.21) and $\hat{\bar{P}}_{i j}$ in $\hat{\bar{E}}_{n}^{\overline{\mathbf{p}}}$ that determine contractions between, respectively, undotted and dotted spinor arguments of different factors of $C\left(Y_{j} ; K \mid x\right)$ and are inherited from the star product (3.5). Since the contribution of $\hat{P}_{i j}$ and $\hat{\bar{P}}_{i j}$-dependent terms is via the exponential it gives rise to a non-polynomial expansion in $p^{i \alpha} p^{j}{ }_{\alpha}$ and $\bar{p}^{i \dot{\alpha}} \bar{p}_{\dot{\alpha}}^{j}$ and, hence, via (2.4) and (2.6), to nonlocal expansion in space-time derivatives. In all available examples nonlinear corrections to HS equations have the form (3.19), (3.21) where at least one of the coefficients $\hat{P}_{i j}(\tau)$ and $\hat{\bar{P}}_{i j}(\bar{\tau})$ is non-zero. This is a manifestation of the fact that in presence of an infinite tower of HS fields the full theory must contain infinite tower of higher derivatives.

A less trivial question is on the locality of vertices involving particular spins $s_{1}, \ldots, s_{n}$. In accordance with $(2.10)$, one-forms $\omega(y, \bar{y} \mid x)$ contain a finite number of components (hence, derivatives of the primaries $\phi_{s}$ ) for a fixed spin $s$. As a result, whether a vertex is local or not depends on the contractions between zero-forms $C(y, \bar{y} ; K \mid x)$. For fixed 
helicities, the degree in $y_{i}$ variables in $C\left(Y_{i} ; K \mid x\right)$ is related to that in $\bar{y}_{i}$. In that case the degree in $p^{i \alpha} p_{\alpha}^{j}$ gets related to that in $\bar{p}^{i \dot{\alpha}} \bar{p}^{j} \dot{\alpha}$ in a particular vertex. As a result, for vertices with fixed spins, polynomiality in $p^{i \alpha} p_{\alpha}^{j}$ implies polynomiality in $\bar{p}^{i \dot{\alpha}} \bar{p}^{j} \dot{\alpha}$ and vice versa. Hence spin-locality for any fixed set of spins is achieved if at least one of the coefficients $\hat{P}_{i j}$ or $\hat{\bar{P}}_{i j}$ is zero for any $i, j .^{2}$ If true in all orders, this implies all-order spin-locality of HS equations. In [9] and [1] it was shown that this can be achieved for all $\omega C^{2}$ and $\omega^{2} C^{2}$ vertices, respectively.

The notion of ultra-locality was originally introduced in [9] as the property that in addition to being free of non-polynomial terms in $\hat{P}_{i j}$ or $\hat{\bar{P}}_{i j}$ the arguments of $C$ in the vertex are free from the $y$ or $\bar{y}$ variables, respectively. The argument of [9] was that if the vertices contain the $Y$-dependence in the lowest orders, their star products will give nonlocal contribution at higher orders. Let us now explain why the definition of ultra-locality of [9] matches that of section 3.4 of this paper. This is again because, in accordance with $(2.10)$, the spin of a constituent field $C(y, \bar{y} ; K)$ is proportional to the difference between the numbers of dotted and undotted indices. Consider for definiteness the holomorphic sector of undotted variables. If zero-forms $C$ in a vertex are free both of the contractions of spinor indices between themselves (i.e. of $\hat{P}_{i j}$ ) and of the $y$-dependence, the undotted spinor indices can only be contracted with those of the one-forms $\omega(Y)$ that, by (2.10), contain at most a finite number of indices for a fixed spin of $\omega(Y)$. The number of anti-holomorphic variables $\bar{y}$ in the arguments of spin- $s_{i}$ zero-forms $C_{s_{i}}$ is then limited by spins in the vertex (including spins of the one-forms) hence remaining finite for any set of spins in the vertex. This implies that the spin of l.h.s. of (3.3) is limited for any given set of spins of the constituent fields hence implying ultra-locality of the vertex in the sense of section 3.4.

It should be stressed that spin-local or ultra-local unfolded equations in which spinlocality or ultra-locality are defined directly in the spinor space as suggested in [9] and in this paper contain full information about HS theory allowing to do computations directly in the spinor space as was for instance demonstrated in [38] where the boundary OPE was computed this way.

\section{Nonlinear higher-spin equations}

$4 d$ nonlinear HS equations [4] have the form

$$
\begin{aligned}
\mathrm{d}_{x} \mathcal{W}+\mathcal{W} * \mathcal{W} & =i\left(\theta^{A} \theta_{A}+\eta B * \gamma+\bar{\eta} B * \bar{\gamma}\right), \\
\mathrm{d}_{x} B+\mathcal{W} * B-B * \mathcal{W} & =0
\end{aligned}
$$

where

$$
\gamma=\theta^{\alpha} \theta_{\alpha} \kappa k, \quad \bar{\gamma}=\bar{\theta}^{\dot{\alpha}} \bar{\theta}_{\dot{\alpha}} \bar{\kappa} \bar{k} .
$$

$\mathcal{W}$ and $B$ are fields of the theory which depend both on space-time coordinates $x^{n}$ and on twistor-like variables $Y^{A}=\left(y^{\alpha}, \bar{y}^{\dot{\alpha}}\right)$ and $Z^{A}=\left(z^{\alpha}, \bar{z}^{\dot{\alpha}}\right)$. It is convenient to introduce

\footnotetext{
${ }^{2}$ Equivalently, the spin-locality condition in $4 d$ HS theory is that the rank of the second derivative matrices contracting indices between any pair of zero-forms $C\left(Y_{j}\right)$ does not exceed 2 .
} 
anticommuting $Z$-differentials $\theta^{A}, \theta^{A} \theta^{B}=-\theta^{B} \theta^{A}$. $B$ is a zero-form, while $\mathcal{W}$ is the oneform with respect to both $d x^{n}$ and $\theta^{A}$ differentials, i.e. $\mathcal{W}=(W, S)$, where $W(Z ; Y ; K \mid x)$ is a space-time one-form, while $S=\theta^{A} S_{A}(Z ; Y ; K \mid x)$. As a result, equation eq. (4.1) contains three equations

$$
\begin{aligned}
\mathrm{d}_{x} W+W * W & =0, \\
\mathrm{~d}_{x} S+W * S+S * W & =0, \\
S * S & =i\left(\theta^{A} \theta_{A}+\eta B * \gamma+\bar{\eta} B * \bar{\gamma}\right),
\end{aligned}
$$

while equation eq. (4.2) gives

$$
\begin{aligned}
\mathrm{d}_{x} B+W * B-W * B & =0, \\
S * B & =B * S .
\end{aligned}
$$

The $Y$ and $Z$ variables provide a realization of HS algebra through the noncommutative associative star product $*$ acting on functions of two spinor variables

$$
(f * g)(Z ; Y)=\int \frac{d^{4} U d^{4} V}{(2 \pi)^{4}} \exp \left[i U^{A} V^{B} C_{A B}\right] f(Z+U ; Y+U) g(Z-V ; Y+V) .
$$

1 is unity of the star-product algebra, i.e. $f * 1=1 * f=f$. Star product (4.9) provides a particular realization of the Weyl algebra

$$
\left[Y_{A}, Y_{B}\right]_{*}=-\left[Z_{A}, Z_{B}\right]_{*}=2 i C_{A B}, \quad\left[Y_{A}, Z_{B}\right]_{*}=0, \quad[a, b]_{*}:=a * b-b * a .
$$

The Klein operators satisfy relations analogous to (2.1) with $y^{\alpha} \rightarrow w^{\alpha}=\left(y^{\alpha}, z^{\alpha}, \theta^{\alpha}\right)$, $\bar{y}^{\dot{\alpha}} \rightarrow \bar{w}^{\dot{\alpha}}=\left(\bar{y}^{\dot{\alpha}}, \bar{z}^{\dot{\alpha}}, \bar{\theta}^{\dot{\alpha}}\right)$, which extend the action of the star product to the Klein operators. Decomposing master fields with respect to the Klein-operator parity, $A^{ \pm}(Z ; Y ; K \mid x)=$ $\pm A^{ \pm}(Z ; Y ;-K \mid x)$, HS gauge fields are $W^{+}, S^{+}$and $B^{-}$while $W^{-}, S^{-}$and $B^{+}$describe an infinite tower of topological fields with every $A d S_{4}$ irreducible field describing at most a finite number of degrees of freedom. (For more detail see [4, 37]).

The left and right inner Klein operators

$$
\kappa:=\exp i z_{\alpha} y^{\alpha}, \quad \bar{\kappa}:=\exp i \bar{z}_{\dot{\alpha}} \bar{y}^{\dot{\alpha}},
$$

which enter eq. (4.3), change a sign of undotted and dotted spinors, respectively,

$$
\begin{aligned}
(\kappa * f)(z, \bar{z} ; y, \bar{y}) & =\exp i z_{\alpha} y^{\alpha} f(y, \bar{z} ; z, \bar{y}), \\
(\bar{\kappa} * f)(z, \bar{z} ; y, \bar{y}) & =\exp i \bar{z}_{\dot{\alpha}} \bar{y}^{\dot{\alpha}} f(z, \bar{y} ; y, \bar{z}), \\
\kappa * f(z, \bar{z} ; y, \bar{y}) & =f(-z, \bar{z} ;-y, \bar{y}) * \kappa, \\
\bar{\kappa} * f(z, \bar{z} ; y, \bar{y}) & =f(z,-\bar{z} ; y,-\bar{y}) * \bar{\kappa}, \\
\kappa * \kappa & =\bar{\kappa} * \bar{\kappa}=1, \quad \kappa * \bar{\kappa}=\bar{\kappa} * \kappa,
\end{aligned}
$$

but commute with the differentials $\theta^{A}$. 
A complex parameter $\eta=|\eta| \exp i \varphi, \varphi \in[0, \pi)$, parameterizes a class of pairwise nonequivalent nonlinear HS theories. The cases of $\varphi=0$ and $\varphi=\frac{\pi}{2}$ correspond to so called $A$ and $B$ HS models that respect parity [39]. In the original paper [4] a more general class of models was considered with an arbitrary star-product function $F_{*}(B)=\eta_{1} B+\eta_{2} B * B+\ldots$ in place of the linear one $\eta B$. As argued in [2], the nonlinear terms in $F_{*}(B)$ are essentially non-local and hence have no obvious holographic duals. From the perspective of this paper, the non-locality of these terms is indeed obvious as is briefly discussed in Conclusion.

\section{$5 \quad$ Perturbative analysis}

Perturbative analysis of eqs. (4.1), (4.2) assumes their linearization around some vacuum solution. The simplest one is

$$
W_{0}(Z ; Y ; K \mid x)=w(Y ; K \mid x), \quad S_{0}(Z ; Y ; K \mid x)=\theta^{A} Z_{A}, \quad B_{0}(Z ; Y ; K \mid x)=0,
$$

where $w(Y \mid x)$ is some solution to the flatness condition

$$
\mathrm{d}_{x} w+w * w=0
$$

A flat connection $w(Y \mid x)$, that describes $A d S_{4}$ via (2.9), is bilinear in $Y^{A}$

$$
w(Y \mid x)=-\frac{i}{4}\left(w^{\alpha \beta}(x) y_{\alpha} y_{\beta}+\bar{w}^{\dot{\alpha} \dot{\beta}}(x) \bar{y}_{\dot{\alpha}} \bar{y}_{\dot{\beta}}+2 h^{\alpha \dot{\beta}}(x) y_{\alpha} \bar{y}_{\dot{\beta}}\right) .
$$

Since $S_{0}$ has a trivial star-commutator with the Klein operators $K$, the star-commutator with $S_{0}$ produces De Rham derivative in $Z$-space

$$
\left[S_{0}, F(Z ; Y ; K \mid x)\right]_{*}=-2 i \mathrm{~d}_{Z} F(Z ; Y ; K \mid x), \quad \mathrm{d}_{Z}:=\theta^{A} \frac{\partial}{\partial Z^{A}} .
$$

HS equations reconstruct the dependence on $Z^{A}$ in terms of the zero-form $C(Y ; K \mid x)$ and one-form $\omega(Y ; K \mid x)$ representing the $\mathrm{d}_{Z}$-cohomological parts of $B$ and $\mathcal{W}$, respectively,

$$
\begin{gathered}
B(Z ; Y ; K \mid x)=C(Y ; K \mid x)+\sum_{j=2}^{\infty} B_{j}(Z ; Y ; K \mid x), \\
\mathcal{W}(Z ; Y ; K \mid x)=\omega(Y ; K \mid x)+\sum_{j=1}^{\infty} \mathcal{W}_{j}(Z ; Y ; K \mid x),
\end{gathered}
$$

where zero-forms $B_{j}(Z ; Y ; K \mid x)$ and one-forms $\mathcal{W}_{j}(Z ; Y ; K \mid x)$ are of order $j$ in $\omega$ and $C$ and have zero projections to $\mathrm{d}_{Z}$ cohomology

$$
h_{\mathrm{d}_{Z}}\left(B_{j}(Z ; Y ; K \mid x)\right)=0, \quad h_{\mathrm{d}_{Z}}\left(\mathcal{W}_{j}(Z ; Y ; K \mid x)\right)=0 \quad \forall j
$$

with the projector $h_{\mathrm{d}_{Z}}$ defined within the chosen homotopy procedure as discussed in section 7 . 
The perturbative analysis goes as follows. Suppose that an order- $n$ solution

$$
\begin{aligned}
\mathcal{W}^{\prime(n)}(Z ; Y ; K \mid x) & =\omega(Y ; K \mid x)+\sum_{j=1}^{n} \mathcal{W}_{j}(Z ; Y ; K \mid x) \\
B^{(n)}(Z ; Y ; K \mid x) & =\sum_{j=1}^{n} B_{j}(Z ; Y ; K \mid x), \quad B_{1}(Z ; Y ; K \mid x)=C(Y ; K \mid x)
\end{aligned}
$$

is found. Then, plugging it into equations (4.5), (4.6), (4.8) that contain $S$ gives equations that determine the dependence on $Z$ in the next order while equations (4.4) and (4.7) turn out to be $Z$-independent as a consequence of the consistency of the system. These produce all nonlinear corrections to the unfolded HS equations in the form (3.3), (3.4).

\section{Star-product functions}

To simplify presentation, in this section we confine ourselves to the holomorphic sector of unbarred variables $z^{\alpha}$ and $y^{\alpha}$. Extension to the antiholomorphic sector is straightforward.

\subsection{Higher-spin algebra $\mathcal{H}$}

\subsubsection{Star product}

Analysis of spin-locality is most convenient in terms of functions $f(z, y, \theta)$ of the form [10]

$$
\begin{aligned}
f(z, y, \theta) & =\int d \tau \phi(\tau z,(1-\tau) y, \tau \theta, \tau) \exp \left[i \tau z_{\alpha} y^{\alpha}\right] \\
& \equiv \int d^{2} \tau \delta\left(1-\tau_{1}-\tau_{2}\right) \exp \left[i \tau_{1} z_{\alpha} y^{\alpha}\right] \phi\left(\tau_{1} z, \tau_{2} y, \tau_{1} \theta, \tau_{1}\right),
\end{aligned}
$$

where $\tau$-kernel $\phi$ is defined as

$$
\begin{aligned}
\phi\left(\tau_{1} z, \tau_{2} y, \tau_{1} \theta, \tau_{1}\right) & =\phi^{\mathbf{i}}\left(\tau_{1} z, \tau_{2} y, \tau_{1} \theta, \tau_{1}\right)+\phi^{\mathbf{b}}\left(\tau_{1} z, \tau_{2} y, \tau_{1} \theta, \tau_{1}\right), \\
\phi^{\mathbf{i}}\left(\tau_{1} z, \tau_{2} y, \tau_{1} \theta, \tau_{1}\right) & =\frac{\tau_{2}}{\tau_{1}} \psi\left(\tau_{1} z, \tau_{2} y, \frac{\tau_{1}}{\tau_{2}} \theta, \tau_{1}\right), \\
\phi^{\mathbf{b}}\left(\tau_{1} z, \tau_{2} y, \tau_{1} \theta, \tau_{1}\right) & =\delta\left(\tau_{1}\right) \chi_{0}(y)+\delta\left(1-\tau_{1}\right) \theta^{\alpha} \theta_{\alpha} \chi_{2}(z)
\end{aligned}
$$

with regular functions $\psi\left(w, u, \xi, \tau_{i}\right), \chi_{0}(y)$ and $\chi_{2}(z)$ such that the poles in (6.3) in $\tau_{1}$ and $\tau_{2}$ are fictitious taking into account that $z$ - and $y$ - dependencies are accompanied with $\tau_{1}$ and $\tau_{2}$, respectively:

$$
\begin{aligned}
\psi\left(\tau_{1} z, \tau_{2} y, 0, \tau_{1}\right) & =\tau_{2}\left(\tau_{1} z^{\alpha} \varsigma_{\alpha}\left(\tau_{1} z, \tau_{2} y, \tau_{1}\right)+\tau_{1} \varsigma\left(\tau_{1} z, \tau_{2} y, \tau_{1}\right)\right), \\
\epsilon^{\alpha \beta} \frac{\partial^{2}}{\partial \theta^{\alpha} \partial \theta^{\beta}} \psi\left(\tau_{1} z, \tau_{2} y, \theta, \tau_{1}\right) & =\tau_{1}\left(\tau_{2} y^{\alpha} \zeta_{\alpha}\left(\tau_{1} z, \tau_{2} y, \tau_{1}\right)+\tau_{2} \zeta\left(\tau_{1} z, \tau_{2} y, \tau_{1}\right)\right),
\end{aligned}
$$

where $\varsigma$ and $\zeta$ are regular.

In the sequel we will distinguish between the inner $\tau$-kernels $\phi^{\mathbf{i}}$ (6.3) and boundary ones $\phi^{\mathbf{b}}$ (6.3). Note that the decomposition of $\phi(6.2)$ into inner and boundary parts is not unique due to the freedom in partial integration over $\tau$ (see section 6.2.2). An important 
consequence of (6.2) is that all inner zero-forms in $\theta$ contain a pre-exponential factor of $\tau_{2}$ while all inner two-forms in $\theta$ contain a pre-exponential factor of $\tau_{1}$.

Functions of the form (6.1) belong to the space of fields $\mathcal{H}$ introduced in [10]

$$
\mathcal{H}:=\oplus_{p=0}^{2} \mathcal{H}_{p}
$$

Here $\mathcal{H}_{p}$ is spanned by such $p$-forms in $\theta(6.1)$ that

$$
\lim _{\tau \rightarrow 0} \tau^{1-p+\varepsilon} \phi^{\mathbf{i}}(w, u, \tau \theta, \tau)=0, \quad \lim _{\tau \rightarrow 1}(1-\tau)^{p-1+\varepsilon} \phi^{\mathbf{i}}(w, u, \tau \theta, \tau)=0 \quad \forall \varepsilon>0
$$

The boundary functions $\phi^{\mathbf{b}}$ associated with $\chi_{0}$ and $\chi_{2}$ belong to $\mathcal{H}_{0}$ and $\mathcal{H}_{2}$, respectively.

Space $\mathcal{H}$ has a number of important properties. As shown in [10] and is explained below, it forms an algebra with respect to the star product. To see this it is convenient to use the following formula [10]:

$$
\begin{aligned}
f_{1} * f_{2}= & \frac{1}{(2 \pi)^{2}} \int_{0}^{1} d \tau_{1} \int_{0}^{1} d \tau_{2} \int d^{2} s d^{2} t \exp i\left[\tau_{1} \circ \tau_{2} z_{\alpha} y^{\alpha}+s_{\alpha} t^{\alpha}\right] \\
& \times \phi_{1}\left(\tau_{1}\left(\left(1-\tau_{2}\right) z-\tau_{2} y+s\right),\left(1-\tau_{1}\right)\left(\left(1-\tau_{2}\right) y-\tau_{2} z+s\right), \tau_{1} \theta, \tau_{1}\right) \\
& \times \phi_{2}\left(\tau_{2}\left(\left(1-\tau_{1}\right) z+\tau_{1} y-t\right),\left(1-\tau_{2}\right)\left(\left(1-\tau_{1}\right) y+\tau_{1} z+t\right), \tau_{2} \theta, \tau_{2}\right),
\end{aligned}
$$

where

$$
\tau_{1} \circ \tau_{2}=\tau_{1}\left(1-\tau_{2}\right)+\tau_{2}\left(1-\tau_{1}\right)
$$

The product law $\circ$ is commutative and associative. Note that $0 \leq \tau_{1} \circ \tau_{2} \leq 1$ and $0 \leq$ $1-\tau_{1} \circ \tau_{2} \leq 1$,

$$
1-\tau_{1} \circ \tau_{2}=\tau_{1} \tau_{2}+\left(1-\tau_{1}\right)\left(1-\tau_{2}\right)
$$

This follows from the simple observation that $\tau_{1} \circ \tau_{2}$ and $1-\tau_{1} \circ \tau_{2}$ can be visualized as areas of the diagonal and off-diagonal rectangles in the unite square cut by horizontal and vertical lines going through points with coordinates $\tau_{1}$ and $\tau_{2}$

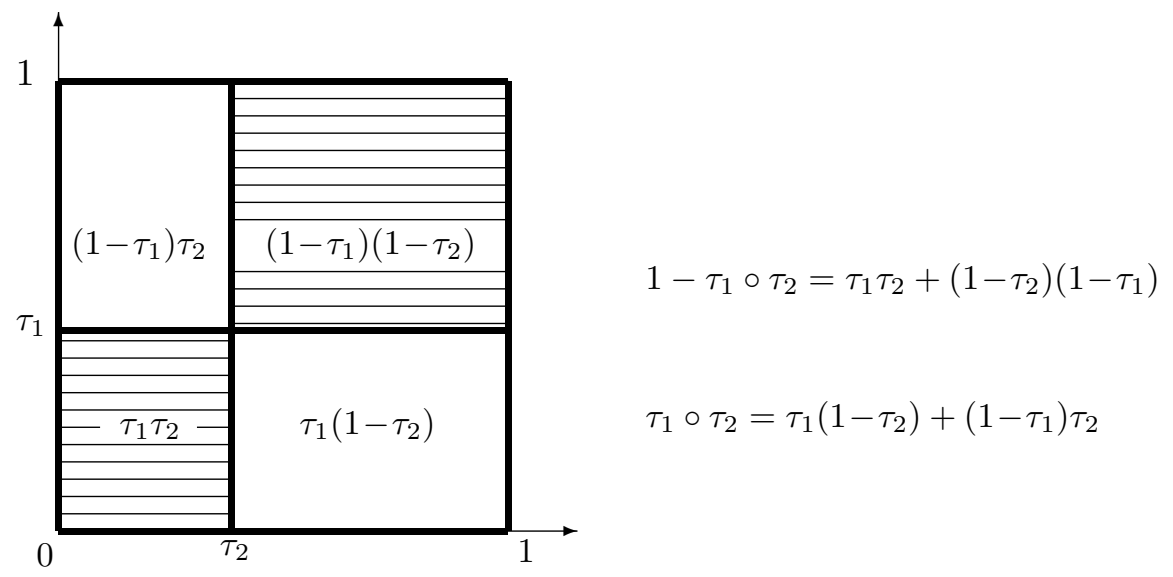




\subsubsection{Inequalities}

Functions

$$
\begin{array}{llll}
\alpha_{11}(\tau):=\frac{\tau_{1} \tau_{2}}{1-\tau_{1} \circ \tau_{2}}, & \alpha_{22}(\tau):=\frac{\left(1-\tau_{1}\right)\left(1-\tau_{2}\right)}{1-\tau_{1} \circ \tau_{2}}, & \alpha_{11}(\tau)+\alpha_{22}(\tau)=1, \\
\alpha_{12}(\tau):=\frac{\tau_{1}\left(1-\tau_{2}\right)}{\tau_{1} \circ \tau_{2}}, & \alpha_{21}(\tau):=\frac{\left(1-\tau_{1}\right) \tau_{2}}{\tau_{1} \circ \tau_{2}}, & & \alpha_{12}(\tau)+\alpha_{21}(\tau)=1
\end{array}
$$

obey obvious inequalities

$$
0 \leq \alpha_{i j}(\tau) \leq 1
$$

Also one can make sure that the following useful inequalities hold by virtue of (6.9)

$$
\tau_{1}\left(1-\tau_{1}\right) \leq \tau_{1} \circ \tau_{2}\left(1-\tau_{1} \circ \tau_{2}\right), \quad \tau_{2}\left(1-\tau_{2}\right) \leq \tau_{1} \circ \tau_{2}\left(1-\tau_{1} \circ \tau_{2}\right) .
$$

Indeed, the first inequality follows from the elementary relation

$$
\tau_{1} \circ \tau_{2}\left(1-\tau_{1} \circ \tau_{2}\right)-\tau_{1}\left(1-\tau_{1}\right)=\tau_{2}\left(1-\tau_{2}\right)\left(1-2 \tau_{1}\right)^{2} \geq 0
$$

Note that from associativity of the product $\circ$ an infinite chain of inequalities follows

$$
\tau_{1} \circ \tau_{2}\left(1-\tau_{1} \circ \tau_{2}\right) \leq \tau_{1} \circ \tau_{2} \circ \tau_{3}\left(1-\tau_{1} \circ \tau_{2} \circ \tau_{3}\right) \leq \ldots
$$

\subsubsection{Class $\mathcal{H}$}

With notations (6.11), $f_{1} * f_{2}$ can be rewritten in the form

$$
\begin{aligned}
f_{1} * f_{2}= & \frac{1}{(2 \pi)^{2}} \int_{0}^{1} d \tau_{1} \int_{0}^{1} d \tau_{2} \int_{0}^{1} d \tau_{1,2} \delta\left(\tau_{1,2}-\tau_{1} \circ \tau_{2}\right) \int d^{2} s d^{2} t \exp i\left[\tau_{1,2} z_{\alpha} y^{\alpha}+s_{\alpha} t^{\alpha}\right] \\
& \phi_{1}\left(\alpha_{12} \tau_{1,2} z-\alpha_{11}\left(1-\tau_{1,2}\right) y+\tau_{1} s, \alpha_{22}\left(1-\tau_{1,2}\right) y-\alpha_{21} \tau_{1,2} z+\left(1-\tau_{1}\right) s, \tau_{1} \theta, \tau_{1}\right) \\
& \phi_{2}\left(\alpha_{21} \tau_{1,2} z+\alpha_{11}\left(1-\tau_{1,2}\right) y-\tau_{2} t, \alpha_{22}\left(1-\tau_{1,2}\right) y+\alpha_{12} \tau_{1,2} z+\left(1-\tau_{2}\right) t, \tau_{2} \theta, \tau_{2}\right) .
\end{aligned}
$$

For instance consider $f_{1}$ and $f_{2}$ with inner $\tau$-kernels (6.3). From (6.15) it follows that $f_{1}^{\mathbf{i}} * f_{2}^{\mathbf{i}}$ is also of the form (6.1) with inner $\tau$-kernel. (The dependence on $\alpha_{i j}\left(\tau_{k}\right)$ in (6.15) does not affect this conclusion thanks to inequalities (6.12).) Moreover, using that [10]

$$
\int_{0}^{1} d \tau_{1} \int_{0}^{1} d \tau_{2} \delta\left(\tau-\tau_{1} \circ \tau_{2}\right)=-\frac{1}{2} \log \left((1-2 \tau)^{2}\right),
$$

one finds following the same reference that the class of functions (6.2) remains invariant under the star product because $-\log \left((1-2 \tau)^{2}\right)$ has simple zeros both at $\tau \rightarrow 0$ and at $\tau \rightarrow 1$. Let us stress that though formula (6.3) contains negative powers of $\tau$ or $1-\tau$, r.h.s. of (6.15) contains no divergencies since, as a consequence of (6.5), the negative powers of $\tau$ and $1-\tau$ are compensated by the $\tau$-dependence of the $z$ - or $y$-dependent terms.

Formula (6.15) simplifies if at least one of functions $f_{1}, f_{2}$ has a boundary $\tau$-kernel. Straightforwardly one can make sure that

$$
f_{1}^{\mathbf{b}} * f_{2}^{\mathbf{b}}=f_{1,2}^{\mathbf{b}}, \quad f_{1}^{\mathbf{i}} * f_{2}^{\mathbf{b}}=f_{1,2}^{\mathbf{i}}, \quad f_{1}^{\mathbf{b}} * f_{2}^{\mathbf{i}}=f_{1,2}^{\mathbf{i}} .
$$


In [10], a subalgebra $\mathcal{H}^{\text {loc }} \subset \mathcal{H}$ was identified such that its elements have a milder dependence at $1-\tau$. In this paper we find it convenient to denote the same algebra $\mathcal{H}^{0+}$. Namely, for $f \in \mathcal{H}_{p}^{0+}$ of the form (6.1) the condition

$$
f \in \mathcal{H}_{p}^{0+}: \quad \exists \varepsilon>0: \quad \lim _{\tau \rightarrow 1}(1-\tau)^{p-1-\varepsilon} \phi(w, u, \tau \theta, \tau)=0
$$

is obeyed. This algebra has a number of interesting properties and was interpreted in [10] as the algebra of local field redefinitions in the theory. Its interpretation in this paper is similar.

\subsubsection{Ideal $\mathcal{I}$}

The new important point not discussed in [10] is that $\mathcal{H}$ contains an ideal $\mathcal{I}$ spanned by functions that have a polynomially softer behavior of $\tau$-kernels both at $\tau \rightarrow 0$ and at $\tau \rightarrow 1$. Namely,

$$
f \in \mathcal{I}: \quad \exists \varepsilon>0: \quad \lim _{\tau \rightarrow 0} \tau^{1-p-\varepsilon} \phi(w, u, \tau \theta, \tau)=0, \quad \lim _{\tau \rightarrow 1}(1-\tau)^{p-1-\varepsilon} \phi(w, u, \tau \theta, \tau)=0 .
$$

Note that the boundary functions with $\tau$-kernels $\phi^{\mathbf{b}}(6.4)$ do not belong to $\mathcal{I}$.

To show that $\mathcal{I}$ is a two-sided ideal of $\mathcal{H}$ we use formula (6.8). Let $f_{1} \in \mathcal{H}, f_{2} \in \mathcal{I}$. Every element of $\mathcal{I}$ contains an additional factor of $\tau^{\varepsilon^{\prime}}(1-\tau)^{\varepsilon^{\prime}}$ with some $\varepsilon^{\prime}>0$. Hence, the product (6.8) contains an additional factor of

$$
a\left(\tau_{2}\right)=\tau_{2}^{\varepsilon^{\prime}}\left(1-\tau_{2}\right)^{\varepsilon^{\prime}}
$$

By virtue of (6.13)

$$
a\left(\tau_{2}\right) \leq\left(\tau_{1} \circ \tau_{2}\left(1-\tau_{1} \circ \tau_{2}\right)\right)^{\varepsilon^{\prime}} .
$$

This implies that $f_{1} * f_{2} \in \mathcal{I}$. Thus $\mathcal{I}$ is a left ideal in $\mathcal{H}$. The proof that $\mathcal{I}$ is also a right, and, hence, two-sided ideal is analogous. To complete the proof, one has to check this property for the boundary terms (6.4). This is elementary as well by virtue of (6.8).

\subsection{5 $\mathcal{H}^{0+}$ and $\mathcal{H}^{+0}$}

Elements of $\mathcal{H}_{p}^{0+}$ obey condition (6.18). Analogously, we define $\mathcal{H}_{p}^{+0}$ as the class of functions obeying

$$
f \in \mathcal{H}_{p}^{+0}: \quad \exists \varepsilon>0: \quad \lim _{\tau \rightarrow 0} \tau^{1-p-\varepsilon} \phi(w, u, \theta, \tau)=0 .
$$

For boundary terms (6.4) we assign

$$
\chi_{0}(y) \in \mathcal{H}_{0}^{0+}, \quad \exp \left[i z_{\alpha} y^{\alpha}\right] \chi_{2}(z) \theta^{\alpha} \theta_{\alpha} \in \mathcal{H}_{2}^{+0} .
$$

Clearly,

$$
\mathcal{I}=\mathcal{H}^{+0} \cap \mathcal{H}^{0+}, \quad \mathcal{H}^{0+}:=\sum_{p=0}^{2} \mathcal{H}_{p}^{0+}, \quad \mathcal{H}^{+0}:=\sum_{p=0}^{2} \mathcal{H}_{p}^{+0} .
$$

It is not difficult to make sure that

$$
\begin{array}{ll}
\mathcal{H}^{0+} * \mathcal{H}^{0+} \subset \mathcal{H}^{0+}, & \mathcal{H}^{+0} * \mathcal{H}^{+0} \subset \mathcal{H}^{0+}, \\
\mathcal{H}^{0+} * \mathcal{H}^{+0} \subset \mathcal{H}^{+0}, & \mathcal{H}^{+0} * \mathcal{H}^{0+} \subset \mathcal{H}^{+0} .
\end{array}
$$


These relations are in agreement with the facts that $\mathcal{H}^{0+}$ forms a subalgebra of $\mathcal{H}$ and $\mathcal{I}$ forms a two-sided ideal of $\mathcal{H}$.

Any $f(z, y, \theta) \in \mathcal{H}$ can be decomposed as

$$
f(z, y, \theta)=f^{0+}(z, y, \theta)+f^{+0}(z, y, \theta), \quad f^{0+}(z, y, \theta) \in \mathcal{H}^{0+}, \quad f^{+0}(z, y, \theta) \in \mathcal{H}^{+0} .
$$

This is achieved by rewriting (6.1) in the form

$$
f(z, y, \theta)=\int d_{+}^{2} \tau \delta\left(1-\tau_{1}-\tau_{2}\right)\left(\tau_{1}+\tau_{2}\right) \exp \left[i \tau_{1} z_{\alpha} y^{\alpha}\right] \phi\left(\tau_{1} z, \tau_{2} y, \tau_{1} \theta, \tau_{1}\right)
$$

giving

$$
\begin{aligned}
& f^{0+}(z, y, \theta)=\int d_{+}^{2} \tau \delta\left(1-\tau_{1}-\tau_{2}\right) \tau_{2} \exp \left[i \tau_{1} z_{\alpha} y^{\alpha}\right] \phi\left(\tau_{1} z, \tau_{2} y, \tau_{1} \theta, \tau_{1}\right) \\
& f^{+0}(z, y, \theta)=\int d_{+}^{2} \tau \delta\left(1-\tau_{1}-\tau_{2}\right) \tau_{1} \exp \left[i \tau_{1} z_{\alpha} y^{\alpha}\right] \phi\left(\tau_{1} z, \tau_{2} y, \tau_{1} \theta, \tau_{1}\right)
\end{aligned}
$$

Note that plugging repeatedly $\tau_{1}+\tau_{2}$ into these formulae and discarding elements of the ideal one can reach any powers of $\tau_{2}$ in (6.29) or $\tau_{1}$ in (6.30). Moreover, discarding terms in the ideal $\mathcal{I}$ one arrives at

$$
\begin{aligned}
& f^{0+}(z, y, \theta) \simeq \int_{0}^{\varepsilon} d \tau \exp \left[i \tau z_{\alpha} y^{\alpha}\right] \phi(\tau z, y, \tau \theta, \tau) \\
& f^{+0}(z, y, \theta) \simeq \int_{1-\varepsilon}^{1} d \tau \exp \left[i \tau z_{\alpha} y^{\alpha}\right] \phi(z,(1-\tau) y, \theta, \tau)
\end{aligned}
$$

with any $\varepsilon>0$ where equivalence $\simeq$ is up to terms in $\mathcal{I}$. Indeed, all terms resulting from the integration over $\tau$ in the region disconnected from 0 and 1 belong to $\mathcal{I}$.

\subsection{Invariant operations}

In this section we consider two more operations that map $\mathcal{H}$ to itself.

\subsection{1 $\gamma$ maps}

Operator $\gamma$ (4.3) belongs to $\mathcal{H}$ and, hence,

$$
\gamma * f \in \mathcal{H}, \quad f * \gamma \in \mathcal{H} \quad \forall f \in \mathcal{H} .
$$

This is because the multiplication with $\gamma$ adds two powers of $\theta$ due to multiplication with $\theta^{\alpha} \theta_{\alpha}$ and exchanges $z$ and $y$ simultaneously replacing $\tau \rightarrow 1-\tau$ as a consequence of (4.12), (4.13). The star product with $\gamma$ maps zero-forms in $\theta$ to two-forms.

A less obvious fact is that star multiplication with $\gamma$ admits inverse $\gamma^{-1}$

$$
\gamma^{-1}(f):=\frac{1}{2} \epsilon^{\alpha \beta} \frac{\partial^{2}}{\partial \theta^{\alpha} \partial \theta^{\beta}} k * \kappa * f(z, y, k, \theta \mid x)
$$

that leaves invariant class $\mathcal{H}$

$$
\gamma^{-1}(f) \in \mathcal{H} \quad \forall f \in \mathcal{H}
$$


Since the multiplication by $\gamma$ and application of $\gamma^{-1}$ swaps $\tau \leftrightarrow 1-\tau$ both of these operations swap $\mathcal{H}^{0+}$ and $\mathcal{H}^{+0}$

$$
\begin{aligned}
\gamma * \mathcal{H}_{0}^{0+} & \subset \mathcal{H}_{2}^{+0}, & \gamma * \mathcal{H}_{0}^{+0} & \subset \mathcal{H}_{2}^{0+}, \\
\gamma^{-1}\left(\mathcal{H}_{2}^{0+}\right) & \subset \mathcal{H}_{0}^{+0}, & \gamma^{-1}\left(\mathcal{H}_{2}^{+0}\right) & \subset \mathcal{H}_{0}^{0+}
\end{aligned}
$$

As a consequence of (6.24) both of them leave ideal $\mathcal{I}$ invariant

$$
\gamma * \mathcal{I} \subset \mathcal{I}, \quad \gamma^{-1}(\mathcal{I}) \subset \mathcal{I}
$$

\subsubsection{Integration by parts}

Analysis of HS field equations sometimes involves integration by parts over the homotopy integration parameters. It is convenient to eliminate a pre-exponential factor of $z_{\alpha} y^{\alpha}$ by partial integration over the homotopy parameter as resulting from the $\frac{\partial}{\partial \tau}$ derivative of the exponential in (6.1). It is important to make sure that this operation leaves invariant classes $\mathcal{H}^{0+}$ and $\mathcal{H}^{+0}$.

Consider the following element of $\mathcal{H}$

$$
f(z, y, \theta)=\int d \tau \theta(\tau) \theta(1-\tau)(1-\tau)^{2} i z_{\alpha} y^{\alpha} \exp \left[i \tau z_{\alpha} y^{\alpha}\right] \psi\left(\tau z,(1-\tau) y, \frac{\tau}{1-\tau} \theta, \tau\right) .
$$

It can be represented as

$$
f(z, y, \theta)=-\int d \tau \frac{\partial}{\partial \tau}\left[\theta(\tau) \theta(1-\tau)(1-\tau)^{2} \psi\left(\tau z,(1-\tau) y, \frac{\tau}{1-\tau} \theta, \tau\right)\right] \exp \left[i \tau z_{\alpha} y^{\alpha}\right]
$$

giving

$$
f(z, y, \theta)=f^{0+}(z, y, \theta)+f^{+0}(z, y, \theta)
$$

where

$$
\begin{aligned}
f^{0+}(z, y, \theta)= & -\psi(0, y, 0,0) \\
& -\int d \tau_{1} d \tau_{2} \theta\left(\tau_{1}\right) \theta\left(\tau_{2}\right) \delta\left(1-\tau_{1}-\tau_{2}\right) \tau_{2}^{2} \frac{\partial}{\partial \tau_{1}}\left[\psi\left(\tau_{1} z, \tau_{2} y, \frac{\tau_{1}}{\tau_{2}} \theta, \tau_{1}\right)\right] \exp \left[i \tau_{1} z_{\alpha} y^{\alpha}\right], \\
f^{+0}(z, y, \theta)= & \left.\tau_{2}^{2} \psi\left(z, 0, \tau_{2}^{-1} \theta, 1\right)\right|_{\tau_{2}=0} \\
& +\int d \tau_{1} d \tau_{2} \theta\left(\tau_{1}\right) \theta\left(\tau_{2}\right) \delta\left(1-\tau_{1}-\tau_{2}\right) \frac{\partial}{\partial \tau_{2}}\left[\tau_{2}^{2} \psi\left(\tau_{1} z, \tau_{2} y, \frac{\tau_{1}}{\tau_{2}} \theta, \tau_{1}\right)\right] \exp \left[i \tau_{1} z_{\alpha} y^{\alpha}\right] .
\end{aligned}
$$

If $f(z, y, \theta) \in \mathcal{H}^{+0}$ then the boundary part of $f^{0+}(6.42)$ is zero, while the inner one $\mathcal{H}^{+0}$ contains an additional degree of $\tau_{1}$. Analogously, if $f(z, y, \theta) \in \mathcal{H}^{0+}$ then $f^{+0}(6.43)$ contains an additional degree of $\tau_{2}$. As a result, the partial integration over the homotopy parameter preserves the classes $\mathcal{H}^{0+}$ and $\mathcal{H}^{+0}$ as well as the ideal $\mathcal{I}$ allowing to freely integrate by parts within a given class. 


\section{Shifted homotopy}

\subsection{General setup}

To eliminate $Z$-variables one has to repeatedly solve equations of the form

$$
\mathrm{d}_{Z} f(Z ; Y ; K \mid x)=g(Z ; Y ; K \mid x)
$$

resulting from equations (4.5), (4.6), (4.8) that contain $S$. Here $g(Z ; Y ; K \mid x)$ is built from already determined lower-order fields $B_{j}(5.5)$ and $W_{j}$ (5.6). Consistency of HS equations guarantees formal consistency of eq. (7.1)

$$
\mathrm{d}_{Z} g(Z ; Y ; K \mid x)=0 .
$$

Given homotopy operator $\partial$

$$
\partial^{2}=0
$$

the operator

$$
A:=\left\{\mathrm{d}_{Z}, \partial\right\}
$$

obeys

$$
\left[\mathrm{d}_{Z}, A\right]=0, \quad[\partial, A]=0 .
$$

For diagonalizable $A$, the standard Homotopy Lemma states that cohomology $H_{\mathrm{d}_{Z}}$ of $\mathrm{d}_{Z}$ is in the kernel of $A$

$$
H_{\mathrm{d}_{Z}} \subset \operatorname{Ker} A .
$$

In this case, it is possible to define such projector $h$ to $\operatorname{Ker} A$

$$
h^{2}=h
$$

and the operator $A^{*}$ that

$$
\left[h, \mathrm{~d}_{Z}\right]=[h, \partial]=0, \quad A^{*} A=A A^{*}=I d-h .
$$

The contracting homotopy operator

$$
\triangle:=A^{*} \partial=\partial A^{*}
$$

gives the resolution of identity

$$
\left\{\mathrm{d}_{Z}, \triangle\right\}+h=I d
$$

allowing to find a solution to equation (7.1) with $\mathrm{d}_{Z}$-closed $g$ outside $H_{\mathrm{d}_{Z}}$ (i.e. obeying $\hat{h} g=0)$ in the form

$$
f=\Delta g+\mathrm{d}_{Z} \epsilon+c,
$$

where an exact part $\mathrm{d}_{Z} \epsilon$ and $c \in H_{\mathrm{d}_{Z}}$ remain undetermined. These describe solutions to the homogeneous equation (7.1) with $g=0$.

The form of the resulting solutions depends on a chosen contracting homotopy $\Delta$. The freedom in this choice affects both the $\mathrm{d}_{z}$-exact and cohomological terms in (7.11). 
The freedom in $\epsilon$ affects the form of gauge transformations while the form of $c(\omega, C)$ induces perturbatively nonlinear field redefinitions. The problem is to single out a specific homotopy procedure that leads to the spin-local form of the field equations at $\epsilon=0, c=0$. In $[2,9]$ we have identified a shifted homotopy that solves the problem in the lowest nontrivial order in the zero-form sector. In [1] and in this paper this construction is extended further to the class of contracting homotopy operators allowing to solve the problem in higher orders as well.

\subsection{Shifted homotopy}

The conventional homotopy operator

$$
\partial=Z^{A} \frac{\partial}{\partial \theta^{A}}
$$

and contracting homotopy

$$
\triangle J(Z ; Y ; \theta)=Z^{A} \frac{\partial}{\partial \theta^{A}} \int_{0}^{1} d t \frac{1}{t} J(t Z ; Y ; t \theta)
$$

were used in the perturbative analysis of HS equations since [4]. Though being simple and looking natural, they are known to lead to non-localities beyond the free field level [8, 12, 40, 41].

An obvious freedom in the definition of homotopy operator (7.12) is to replace $Z^{A}$ by $Z^{A}+a^{A}$ with some $Z$-independent $a^{A}$,

$$
\partial \rightarrow \partial_{a}=\left(Z^{A}+a^{A}\right) \frac{\partial}{\partial \theta^{A}}, \quad \frac{\partial}{\partial Z^{A}}\left(a^{B}\right)=0 .
$$

Contracting homotopy $\triangle_{a}$ and cohomology projector $h_{a}$ act as follows

$$
\triangle_{a} \phi(Z, Y, \theta)=\int_{0}^{1} \frac{d t}{t}(Z+a)^{A} \frac{\partial}{\partial \theta^{A}} \phi(t Z-(1-t) a, t \theta), \quad h_{a} \phi(Z, Y, \theta)=\phi(-a, Y, 0) .
$$

$\triangle_{0}$ is conventional contracting homotopy (7.13). The resolution of identity has standard form

$$
\left\{\mathrm{d}_{Z}, \triangle_{a}\right\}+h_{a}=I d .
$$

For instance, one can set $a^{A}=c Y^{A}$ with some constant $c$. Naively, this exhausts all Lorentz covariant options for $a^{A}$. However $a^{A}$ can also be composed from the derivatives with respect to the arguments of $\omega(Y ; K)$ and $C(Y ; K)$ in $g=g(\omega, C)(7.1)$.

Let

$$
\Phi^{1}(Y ; K)=\omega(Y ; K), \quad \Phi^{0}(Y ; K)=C(Y ; K) .
$$

Various terms on the r.h.s. of HS field equations contain products

$$
\Phi_{n}^{\mathbf{a}}(Y ; K)=\left.\Phi^{a_{1}}\left(Y_{1} ; K\right) \Phi^{a_{2}}\left(Y_{2} ; K\right) \ldots \Phi^{a_{n}}\left(Y_{n} ; K\right)\right|_{Y_{i}=Y}, \quad \mathbf{a}=\left\{a_{1}, \ldots, a_{n}\right\}, \quad a_{i}=0,1 .
$$


These products is useful to treat independently for different orderings of $\omega$ and $C$ since HS equations are known to remain consistent with all fields valued in any associative (say, matrix) algebra [24], in which case the fields are not commuting. This implies that the terms associated with different labels a can be treated as independent.

The simplest option used in $[2,9]$ is

$$
a_{A}^{\mathbf{a}}=c_{0}(\mathbf{a}) Y_{A}+\sum_{j} c_{j}(\mathbf{a}) \partial_{j A}, \quad \mathbf{a}=\left\{a_{1}, \ldots, a_{n}\right\}
$$

where $\partial_{i A}$ is the derivative with respect to the argument of the $i^{\text {th }}$ factor $\Phi^{a_{i}}\left(Y_{i} ; K\right)$. The

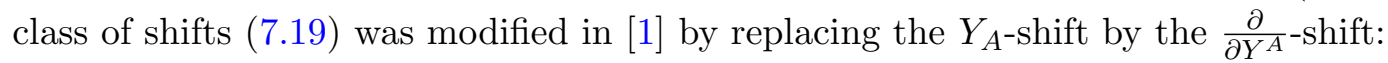

$$
a_{A}^{\mathbf{a}}=i \beta(\mathbf{a}) \frac{\partial}{\partial Y^{A}}+\sum_{j} c_{j}(\mathbf{a}) \partial_{j A}, \quad \mathbf{a}=\left\{a_{1}, \ldots, a_{n}\right\}
$$

Note that it is hard to keep the $Y_{A^{-}}$and $\frac{\partial}{\partial Y^{A}}$-shifts simultaneously because they do not commute and, hence, the resulting shifts $a^{\mathbf{a}}{ }_{A}$ would be noncommuting that is not allowed.

In fact, formula (7.15) with shift (7.20) is not convenient for practical computations. The following integral representation for the shifted contracting homotopy [1] is more useful:

$$
\triangle_{0, \beta} f(z, y, \theta)=\int \frac{d^{2} u d^{2} v}{(2 \pi)^{2}} \exp i v_{\alpha} u^{\alpha} \int_{0}^{1} \frac{d t}{t}(z-u)^{\alpha} \frac{\partial}{\partial \theta^{\alpha}} f(t z+(1-t) u, \beta v+y, t \theta) .
$$

(To simplify formulae we confine ourselves to the sector of left spinors with undotted indices.) More generally, for any $z, y$-independent spinor $q$,

$$
\triangle_{q, \beta} f(z, y, \theta):=\int \frac{d^{2} u d^{2} v}{(2 \pi)^{2}} \exp i v_{\alpha} u^{\alpha} \int_{0}^{1} \frac{d t}{t}(z-u+q)^{\alpha} \frac{\partial}{\partial \theta^{\alpha}} f(t z+(1-t)(u-q), \beta v+y, t \theta) .
$$

This shifted contracting homotopy obeys resolution of identity (7.16) with the cohomology projector

$$
h_{q, \beta}(f(z, y, \theta))=\int \frac{d^{2} u d^{2} v}{(2 \pi)^{2}} \exp i v_{\alpha} u^{\alpha} f(u-q, \beta v+y, 0)
$$

Note that application of formulae (7.21) and (7.23) to functions (6.1) leads to the Gaussian integration over $u^{\alpha}$ and $v^{\alpha}$ which, in turn, generates nontrivial Jacobian in the integration measure, that is hard to obtain in the differential definition (7.20). This Jacobian plays crucial role in the analysis of spin-locality in [1] and in this paper.

Formulae (7.21) and (7.23) can be easily extended to the class of homotopies (7.20) containing shifts of arguments of various fields $f(z, y, \theta)$ is built of. For instance, for

$$
f(z, y, \theta)=\left.F\left(z, y, \frac{\partial}{\partial y_{i}}\right) \Phi\left(y_{1}, K\right) \ldots \Phi\left(y_{k}, K\right)\right|_{y_{i}=0}
$$

appropriate modifications of (7.21) and (7.23) result from the replacement of $C\left(y_{1}\right) \ldots C\left(y_{k}\right)$ by $C\left(c_{1} v+y_{1}\right) \ldots C\left(c_{k} v+y_{k}\right)$. 
Formula (7.23) yields for $f(z, y, \theta)(6.1)$

$$
\begin{aligned}
h_{(1-\beta) q, \beta}(f)= & \int_{0}^{1} d \tau \zeta^{-2} \int \frac{d^{2} u d^{2} v}{(2 \pi)^{2}} \exp i\left[v_{\alpha} u^{\alpha}+\tau(1-\beta) \zeta^{-1} y_{\alpha} q^{\alpha}\right] \\
& \phi\left(\tau(\beta u-(1-\beta) q) \zeta^{-1},(1-\tau)\left(v+y \zeta^{-1}\right), 0, \tau\right),
\end{aligned}
$$

where

$$
\zeta:=(1-\beta \tau) .
$$

Note that we use a normalized shift $q(1-\beta)$, that naturally appears in the star-exchange procedure [1] (see also appendix A).

The contracting homotopy with $q=0$ was presented in [1]. Derivation of the expression for contracting homotopy with any $q$ sketched in appendix B yields

$$
\begin{aligned}
\triangle_{(1-\beta) q, \beta}(f)= & \int \frac{d^{2} u d^{2} v}{(2 \pi)^{2}} \int d_{+}^{3} \tau \delta\left(1-\sum_{i=1}^{3} \tau_{i}\right)\left[\frac{(1-\beta) \tau_{1}}{1-\beta\left(1-\tau_{2}\right)}\right]^{p-1} \\
& \exp i\left[v_{\alpha} u^{\alpha}+\tau_{1} z_{\alpha} y^{\alpha}-\tau_{2} q_{\alpha} y^{\alpha}\right] \frac{\left(1-\beta \tau_{1}\right)(z+q)^{\beta}-\beta \tau_{3}(u+q)^{\beta}}{1-\beta\left(1-\tau_{2}\right)} \frac{\partial}{\partial \theta^{\beta}} \\
& \phi\left(\tau_{1} z+\frac{\tau_{2} \tau_{3} \beta}{1-\beta\left(1-\tau_{2}\right)} u-\tau_{2} q, v+\tau_{3} y, \theta, \frac{1-\tau_{3}-\beta \tau_{1}}{1-\beta\left(1-\tau_{2}\right)}\right)
\end{aligned}
$$

where

$$
d_{+}^{3} \tau:=d \tau_{1} d \tau_{2} d \tau_{3} \theta\left(\tau_{1}\right) \theta\left(\tau_{2}\right) \theta\left(\tau_{3}\right), \quad \theta(\tau)=1(0) \quad \text { if } \quad \tau \geq 0(\tau<0)
$$

and $p$ is the degree of $f$ in $\theta$ :

$$
f(w, u, \mu \theta, \tau)=\mu^{p} f(w, u, \theta, \tau) .
$$

The last argument of $\phi$ in (7.27) results from the change of integration variables (B.7).

For inner functions $\phi^{\mathbf{i}}(6.3)$ the contracting homotopy takes the form

$$
\begin{aligned}
\triangle_{(1-\beta) q, \beta}(f)= & \int \frac{d^{2} u d^{2} v}{(2 \pi)^{2}} \int d_{+}^{3} \tau \delta\left(1-\sum_{i=1}^{3} \tau_{i}\right) \exp i\left[v_{\alpha} u^{\alpha}+\tau_{1} z_{\alpha} y^{\alpha}-\tau_{2} q_{\alpha} y^{\alpha}\right] \\
& \left(\frac{\tau_{1}}{\tau_{3}}\right)^{p-1} \frac{\left(1-\beta \tau_{1}\right)(z+q)^{\beta}-\beta \tau_{3}(u+q)^{\beta}}{\left(1-\beta \tau_{1}+\tau_{3}\right)} \frac{\partial}{\partial \theta^{\beta}} \\
& \psi\left(\tau_{1} z+\frac{\tau_{2} \tau_{3} \beta}{1-\beta\left(1-\tau_{2}\right)} u-\tau_{2} q, v+\tau_{3} y, \theta, \frac{1-\tau_{3}-\beta \tau_{1}}{1-\beta\left(1-\tau_{2}\right)}\right) .
\end{aligned}
$$

To simplify formulae in the sequel we will use notations

$$
\triangle_{q, \beta}^{\prime}:=\triangle_{(1-\beta) q, \beta}, \quad h_{q, \beta}^{\prime}:=h_{(1-\beta) q, \beta} .
$$

Formulae (7.25), (7.27) and (7.30) contain nontrivial prefactors and rational dependence on the integration homotopy parameters $\tau$ resulting from the substitution of the dependence on $u$ and $v$ into the exponential factor in (6.1). The final expressions are well defined for

$$
-\infty<\beta<1
$$


In particular, the potential divergency due to the factor of $\tau^{-1}$ in (6.2) does not contribute in (7.25) because of the factor of $\tau$ in the first argument of $\phi$. The seeming divergency due to the factor of $\tau_{3}^{-1}$ in (7.30) is compensated due to the second regularity condition (6.5).

Beyond this region, divergencies can appear due to the degeneracy of the quadratic form in the Gaussian integral. At $\beta=0$, these formulae reproduce those of the conventional contracting homotopy introduced in [4] (see also [37]). Let us note that, as shown in [1], the expression $\triangle_{q, \beta}^{\prime}(\gamma)$ is $\beta$-independent

$$
\triangle_{q, \beta}^{\prime}(\gamma)=\triangle_{q, 0}(\gamma)
$$

that, along with star-exchange formulae (see appendix A), imply that the analysis of the $\eta \bar{\eta}$ sector of HS equations turns out to be insensitive to $\beta$ and can be performed in particular at $\beta=0$ as in $[2,9]$. These vertices are also found in [1].

\subsection{Pfaffian Locality Theorem}

A class of shifted contracting homotopies introduced in [2] was shown to reduce the degree of non-locality in all orders of the perturbation theory provided that shifts obey certain conditions prescribed by the Pfaffian Locality Theorem (PLT). Properties of these contracting homotopies were studied in [9] where they were shown to reproduce spin-local lower-order vertex $\Upsilon(\omega, C, C)$ found originally in $[6,8]$ provided that the PLT conditions are respected. Here we extend PLT to the $\beta$-shifted contracting homotopies.

In [2] we have identified odd and even classes of functions as follows. General exponential representation for order- $n$ corrections in the zero-forms $C$ has the form

$$
\left.\sum_{\mathbf{p} \overline{\mathbf{p}}} \int d \tau P_{n}^{\mathbf{p} \overline{\mathbf{p}}} E_{n}^{\mathbf{p} \overline{\mathbf{p}}}(\tau) C\left(Y_{1}\right) \ldots C\left(Y_{n}\right)\right|_{Y_{j}=0}
$$

where $P_{n}^{\mathbf{p} \overline{\mathbf{p}}}$ is some polynomial of $z, y$ and $p^{i}(3.20)$ and their conjugates with coefficients being regular functions of the homotopy parameters $\tau$, and

$$
\begin{aligned}
E_{n}^{\mathbf{p} \overline{\mathbf{p}}} & =E_{n}^{\mathbf{p}} \bar{E}_{n}^{\overline{\mathbf{p}}}, \\
E_{n}^{\mathbf{p}}(T, A, B, P, p \mid z, y) & =\exp i\left(T z_{\gamma} y^{\gamma}-A_{j} p_{\gamma}^{j} z^{\gamma}-B_{j} p_{\gamma}^{j} y^{\gamma}+\frac{1}{2} P_{i j} p^{i \gamma} p^{j} \gamma\right) k^{\mathbf{p}},
\end{aligned}
$$

where $\mathbf{p}=0,1$ and coefficients $T \in \mathbb{C}, A, B \in \mathbb{C}^{n}, P_{i j}=-P_{j i} \in \mathbb{C}^{n} \times \mathbb{C}^{n}$ may be $\tau$-dependent.

In the even class of $k$-equipped exponentials

$$
\mathcal{E}_{n}^{0}: \quad E_{n}^{\mathbf{p}}(T, A, B, P, p \mid z, y), \quad \mathbf{p}=\left.n\right|_{\bmod 2}, \quad n \geq 1
$$

coefficients in (7.35) satisfy

$$
\sum_{j=1}^{n}(-1)^{j} A_{j}=-T, \quad \sum_{j=1}^{n}(-1)^{j} B_{j}=0, \quad \sum_{i=1}^{n}(-1)^{i} P_{i j}=B_{j} .
$$

In the odd class of $k$-equipped exponentials

$$
\mathcal{E}_{n}^{1}: \quad E_{n}^{\mathbf{p}}(T, A, B, P, p \mid z, y), \quad \mathbf{p}=\left.(n+1)\right|_{\bmod 2}, \quad n \geq 0
$$


coefficients obey

$$
\sum_{j=1}^{n}(-1)^{j} A_{j}=0, \quad \sum_{j=1}^{n}(-1)^{j} B_{j}=1-T, \quad \sum_{i=1}^{n}(-1)^{i} P_{i j}=-A_{j} .
$$

The odd and even classes form a $\mathbb{Z}_{2}$-graded algebra with respect to star product

$$
\mathcal{E}_{n}^{j} * \mathcal{E}_{m}^{i} \subseteq \mathcal{E}_{m+n}^{\left.(j+i)\right|_{2}}
$$

in the sense that if conditions (7.37) or (7.39) were respected by the product factors $f$ and $g$, the same conditions of the respective parity will be respected by $f * g$.

Following [2] we consider the action of the contracting homotopy $\triangle_{q_{n}(v), \beta}^{\prime}(7.31)$ with

$$
q_{n}(v)=v_{j} p^{j}, \quad v_{j} \in \mathbb{C}^{n}
$$

on $\phi_{n} E_{n}^{\mathbf{p}}$, where $E_{n}^{\mathbf{p}}$ is some $k$-equipped exponential $(7.35)$, while $\phi(z, y, p, \theta)$ is a preexponential factor containing a finite number of $p^{j}$.

By definition (7.22), performing Gaussian integration with respect to $u$ and $v$ one has

$$
\triangle_{q_{n}(v), \beta}^{\prime} \phi_{n} E_{n}^{\mathbf{p}}(T, A, B, P \mid z, y)=\int_{0}^{1} d \sigma \widetilde{\phi}_{n}(\sigma, z, y, v, \beta, \theta) E_{n}^{\prime \mathbf{p}}\left(T^{\prime}, A^{\prime}, B^{\prime}, P^{\prime} \mid z, y\right)
$$

where $\widetilde{\phi}$ is some pre-exponential factor and

$$
E_{n}^{\prime \mathbf{p}}=\exp i\left(T^{\prime} z_{\gamma} y^{\gamma}-A_{j}^{\prime} p_{\gamma}^{j} z^{\gamma}-B_{j}^{\prime} p_{\gamma}^{j} y^{\gamma}+\frac{1}{2} P_{i j}^{\prime} p^{i \gamma} p_{\gamma}^{j}\right) k^{\mathbf{p}}
$$

with

$$
\begin{aligned}
T^{\prime} & =\sigma T \xi^{-1}, \quad A_{i}^{\prime}=\sigma A_{i} \xi^{-1}, \quad B_{i}^{\prime}=\left(B_{i}+(1-\sigma)(1-\beta) T v_{i}\right) \xi^{-1}, \\
P^{\prime}{ }_{i j} & =P_{i j}+(1-\sigma)(1-\beta)\left(A_{j} v_{i}-A_{i} v_{j}\right) \xi^{-1}-\beta \xi^{-1}(1-\sigma)\left(B_{j} A_{i}-B_{i} A_{j}\right), \\
\xi & =1-(1-\sigma) T \beta .
\end{aligned}
$$

Elementary calculation yields the following

Lemma 3 of [2]: If

$$
\begin{aligned}
\sum_{j=1}^{n}(-1)^{j} v_{j} & =1, \\
E_{n}^{\mathbf{p}}(T, A, B, P \mid z, y) & \in \mathcal{E}_{n}^{1}
\end{aligned}
$$

then $k$-equipped exponential $E_{n}^{\prime \mathrm{p}}\left(T^{\prime}, A^{\prime}, B^{\prime}, P^{\prime}\right)(7.43)$ belongs to $\mathcal{E}_{n}^{1}$ for any $\sigma$ and $\beta$.

Indeed, by virtue of (7.44)-(7.46), coefficients $T^{\prime}, A^{\prime}, B^{\prime}, P^{\prime}$ of $E$ (7.43) can be easily shown to satisfy (7.39) for any $\sigma$ and $\beta$.

Contracting homotopy $\triangle_{q_{n}(v), \beta}^{\prime}$ with $v$ satisfying (7.45) will be called odd. Note that PLT-condition (7.45) coincides with that of [2] obtained at $\beta=0$. 
Analogously, one proves the following

Lemma 4 of [2]: If

$$
\begin{array}{r}
\sum_{j=1}^{n}(-1)^{j} v_{j}=0, \\
E_{n}^{\mathbf{p}}(T, A, B, P, p \mid z, y) \in \mathcal{E}_{n}^{0}
\end{array}
$$

then $E \in \mathcal{E}_{n}^{0}$ for any $\sigma$ and $\beta$.

Contracting homotopy $\triangle_{q_{n}(v), \beta}^{\prime}$ with $v$ obeying (7.47) will be called even. Note that PLT-condition (7.47) coincides with that of [2] in the absence of $y$-shifts.

In [2] we considered the odd class of zero-forms using PLT to show that the final result is spin-local by virtue of $Z$-dominance Lemma stating that since all $Z$-dependent terms should disappear upon reduction to the cohomology sector, the part of the coefficients $P_{i j}$ responsible for contraction of derivatives between different factors of $C$ must vanish as well because they are proportional to the coefficients $A_{i}$ in the $z$-dependent terms in (7.35). In the even case, this argument does not work since $P_{i j}$ is related to the coefficients $B_{i}$ in the $y$-dependent term in (7.35). However, the latter relation is useful again since, as will be shown below, the final result turns out to be $y$-independent in the terms important for the analysis of spin-locality. As a result, as explained in more detail in sections 3.4, 3.6, it becomes not just spin-local, but ultra-local in terminology of [9].

\section{Limiting contracting homotopy and Factorization Lemma}

As argued in [1] to obtain a local frame in the HS theory one has to use contracting homotopy in the limit $\beta \rightarrow-\infty$. Our goal is to analyse when the limit $\beta \rightarrow-\infty$ is well defined. Let us stress that even if it is not, this does not mean that the theory is ill-defined but rather that it is unlikely spin-local since $\beta$ has to be kept finite. In all cases analysed so far this does not happen, however. In section 9.2.3 we formulate a sufficient condition guaranteeing that the limit $\beta \rightarrow-\infty$ is well defined.

\subsection{Limiting contracting homotopy}

To analyse the limit $\beta \rightarrow-\infty$ one has to use the class of functions (6.1). The worst possibility would be if the terms in the arguments of $\phi$ in (7.27) were divergent. Fortunately, this does not happen. Since $\sum_{i=1}^{3} \tau_{i}=1$, the $\beta$-dependent coefficient in $\phi$ does not exceed 1 hence being well defined at $\beta \rightarrow-\infty$. This allows us to take the limit directly in (7.27) to obtain

$$
\begin{aligned}
\triangle_{q,-\infty}^{\prime}(f)= & \int \frac{d^{2} u d^{2} v}{(2 \pi)^{2}} \int d_{+}^{3} \tau \delta\left(1-\sum_{i=1}^{3} \tau_{i}\right)\left[\frac{\tau_{1}}{\tau_{1}+\tau_{3}}\right]^{p-1} \exp i\left[v_{\alpha} u^{\alpha}+\tau_{1} z_{\alpha} y^{\alpha}-\tau_{2} q_{\beta} y^{\beta}\right] \\
& \frac{\tau_{1}\left(z^{\beta}+q^{\beta}\right)+\tau_{3}\left(u^{\beta}+q^{\beta}\right)}{\tau_{1}+\tau_{3}} \frac{\partial}{\partial \theta^{\beta}} \phi\left(\tau_{1} z-\frac{\tau_{2} \tau_{3}}{\tau_{1}+\tau_{3}} u-\tau_{2} q, v+\tau_{3} y, \theta, \frac{\tau_{1}}{\tau_{1}+\tau_{3}}\right) .
\end{aligned}
$$


Analogously, (7.30) gives at $\beta \rightarrow-\infty$

$$
\begin{aligned}
\triangle_{q,-\infty}^{\prime}(f)= & \int \frac{d^{2} u d^{2} v}{(2 \pi)^{2}} \int d_{+}^{3} \tau \delta\left(1-\sum_{i=1}^{3} \tau_{i}\right) \exp i\left[v_{\alpha} u^{\alpha}+\tau_{1} z_{\alpha} y^{\alpha}-\tau_{2} q_{\beta} y^{\beta}\right] \\
& \times\left(\frac{\tau_{1}}{\tau_{3}}\right)^{p-2}\left(\frac{\tau_{1}}{\tau_{3}}\left(z^{\beta}+q^{\beta}\right)+\left(u^{\beta}+q^{\beta}\right)\right) \\
& \times \frac{\partial}{\partial \theta^{\beta}} \psi\left(\tau_{1} z-\frac{\tau_{2} \tau_{3}}{\tau_{1}+\tau_{3}} u-\tau_{2} q, v+\tau_{3} y, \theta, \frac{\tau_{1}}{\tau_{1}+\tau_{3}}\right) .
\end{aligned}
$$

The fate of potential divergency on the r.h.s. of (8.2) due to the factor of $\tau_{3}^{p-2}$ is discussed in sections 9.2.1 and 9.2.3. Note that

$$
\frac{\tau_{1}}{\tau_{1}+\tau_{3}} \leq 1, \quad \frac{\tau_{3}}{\tau_{1}+\tau_{3}} \leq 1
$$

Naively, one might think that $\left(\frac{\tau_{1}}{\tau_{1}+\tau_{3}}\right)^{n}$ behaves as $\tau_{1}^{n}$ at $\tau_{1} \rightarrow 0$. However, this is not the case because of the integration over $\tau_{3}$. Indeed

$$
\begin{gathered}
\int d \tau_{2} d \tau_{3} \delta\left(1-\sum_{i=1}^{3} \tau_{i}\right) \frac{\tau_{1}^{n}}{\left(\tau_{3}+\tau_{1}\right)^{n}}=\int_{0}^{1-\tau_{1}} d \tau_{3} \frac{\tau_{1}^{n}}{\left(\tau_{3}+\tau_{1}\right)^{n}}=\frac{1}{n-1} \tau_{1}\left(1-\tau_{1}^{n-1}\right), \quad n>1 \\
\int d_{+}^{3} \tau \delta\left(1-\sum_{i=1}^{3} \tau_{i}\right) \frac{\tau_{1}}{\left(\tau_{3}+\tau_{1}\right)}=\int_{0}^{1-\tau_{1}} d \tau_{3} \frac{\tau_{1}}{\left(\tau_{3}+\tau_{1}\right)}=-\tau_{1} \log \tau_{1}
\end{gathered}
$$

The common feature of this expressions is that, independently of $n \geq 1$ they have simple zeros both at $\tau_{1}=0$ and at $\tau_{1}=1$. (Logarithmic corrections do not matter in our analysis.) This allows us to estimate behaviour of the integrand of (8.1) at $\tau_{1} \rightarrow 0$ and $\tau_{1} \rightarrow 1$ upon integration over $\tau_{2}$ and $\tau_{3}$. If the factors $\tau_{1}$ and $1-\tau_{1}$ enter explicitly the formulae above give for the leading behaviour at $\tau_{1} \rightarrow 0$ and $\tau_{1} \rightarrow 1$

$$
\int_{0}^{1} \int_{0}^{1} d \tau_{2} d \tau_{3} \tau \delta\left(1-\sum_{i=1}^{3} \tau_{i}\right) \frac{\tau_{1}^{n+m}\left(1-\tau_{1}\right)^{k}}{\left(\tau_{3}+\tau_{1}\right)^{n}}=\alpha(n, m, k) \tau_{1}^{m+1}\left(1-\tau_{1}\right)^{k+1}, \quad n>0,
$$

where $\alpha(n, m, k)$ are some coefficients.

We conclude that expressions

$$
X=\int d_{+}^{3} \tau \delta\left(1-\sum_{i=1}^{3} \tau_{i}\right) \frac{\tau_{1}^{n}}{\left(\tau_{1}+\tau_{3}\right)^{n}} \phi\left(\tau_{1} z,\left(1-\tau_{1}\right) y, \tau_{1}\right) \exp i\left[\tau_{1} z_{\alpha} y^{\alpha}\right]
$$

with $n \geq 1$ behave with respect to $\tau_{1}$ as

$$
X \sim \int_{0}^{1} d \tau_{1} \tau_{1}\left(1-\tau_{1}\right) \phi\left(\tau_{1} z,\left(1-\tau_{1}\right) y, \tau_{1}\right) \exp i\left[\tau_{1} z_{\alpha} y^{\alpha}\right]
$$

i.e. independently of $n, \tau_{3}$-integration adds one power of both $\tau_{1}$ and $1-\tau_{1}$. 


\subsection{Factorization Lemma and limiting cohomology projector}

\subsubsection{Factorization Lemma}

Factorization Lemma states: In the limit $\beta \rightarrow-\infty$, cohomology projector (7.25) gives zero on $\mathcal{H}^{+0}$ :

$$
h_{q,-\infty}^{\prime}\left(\mathcal{H}^{+0}\right)=0 .
$$

Indeed, as shown in [1], typical integrals that appear in the limit $\beta \rightarrow-\infty$ have the form

$$
\lim _{\beta \rightarrow-\infty} \int_{0}^{1} \mathrm{~d} \tau \frac{\beta(\beta \tau)^{m}}{(1-\beta \tau)^{2+n}}, \quad n \geq m .
$$

Obviously, it gives a finite result after the change of variables $\tau \rightarrow \tau^{\prime}=-\beta \tau$. However, if there is an additional factor of $\tau^{\varepsilon}$ in (7.25) and hence (8.10) this is equivalent to the appearance of the factor of $(-\beta)^{-\varepsilon}$ that sends the final result to zero in the limit $\beta \rightarrow-\infty$.

Note that Factorization Lemma provides a simple interpretation of the $Z$-dominance Lemma of [2] which states that if the coefficients in front of the terms in the exponential responsible for contractions between different product factors in (7.18) are dominated by the coefficient in front of $i z_{\alpha} y^{\alpha}$, i.e. $\tau$, then these terms do not contribute to the dynamical equations leading to spin-local field equations. In the setup of this paper this is simply because, being dominated by $\tau$, contractions bring an extra factor of $\tau$ hence belonging to $\mathcal{H}^{+0}$.

\subsubsection{Limiting cohomology projector}

Remarkably, the cohomology projector (7.25) remains finite in the limit $\beta \rightarrow-\infty$. Naively, it gives 0 at $\beta=-\varepsilon^{-1}$ with $\varepsilon \rightarrow 0$ since

$$
\int_{0}^{1} d \tau \frac{1}{(1-\beta \tau)^{2+n}}=-(n+1)^{-1}\left(\frac{\varepsilon^{n+2}}{(\varepsilon+1)^{n+1}}-\frac{\varepsilon^{n+2}}{\varepsilon^{n+1}}\right)=(n+1)^{-1} \varepsilon+O\left(\varepsilon^{2}\right) .
$$

However, this is not the case because, being a zero-form in $\theta, \phi$ in (7.25) contains a factor of $\tau^{-1}$ in front of the $u$-dependent terms. Indeed, by rescaling $v_{\alpha} \rightarrow(1-\tau)^{-1} v_{\alpha}$, $u_{\alpha} \rightarrow(1-\tau) u_{\alpha}$ the coefficient in front of $v$ in the argument of $\phi$ in (7.25) takes the form

$$
\left|\frac{\tau(1-\tau) \beta}{(1-\tau \beta)}\right| \leq 1
$$

As such, it disappears in the limit $\beta \rightarrow-\infty$ by (8.11). However, once one of the factors of $\tau$ is removed, $\beta \frac{(1-\tau)}{(1-\tau \beta)}$ contains an extra factor of $\beta \sim \varepsilon^{-1}$ that precisely compensates the factor of $\varepsilon$ in (8.11) hence yielding a finite result in the limit. Thus, it is crucial that $\phi$ (6.1) is of the form (6.2).

The limit $\beta \rightarrow-\infty$ can be taken directly in (7.25) to obtain for any $f^{\mathbf{i}}$

$$
h_{q,-\infty}^{\prime}\left(f^{\mathbf{i}}\right)=\int_{0}^{1} d \sigma \int \frac{d^{2} u d^{2} v}{(2 \pi)^{2}} \exp i\left[v_{\alpha} u^{\alpha}+\sigma y_{\alpha} q^{\alpha}\right] \phi^{\mathbf{i}}(-\sigma(q+u), v+(1-\sigma) y, 0,0) \text {. }
$$


Indeed, due to (6.2), eq. (7.25) yields for an inner zero-form $\phi^{\mathbf{i}}$

$$
\begin{aligned}
h_{q, \beta}^{\prime}\left(f^{\mathbf{i}}\right)= & \int_{0}^{1} d \tau(1-\beta \tau)^{-2} \int \frac{d^{2} u d^{2} v}{(2 \pi)^{2}} \exp i\left[v_{\alpha} u^{\alpha}\right] \exp i\left((\tau-\beta \tau)(1-\beta \tau)^{-1} y_{\alpha} q^{\alpha}\right) \\
& \phi\left((\tau \beta u-(\tau-\beta \tau) q)(1-\beta \tau)^{-1},(1-\tau)\left(v+y(1-\beta \tau)^{-1}\right), 0, \tau\right) .
\end{aligned}
$$

Using that $\frac{\beta(1-\beta \tau)^{-1}}{\left[1-(1-\beta \tau)^{-1}\right]}=-\frac{1}{\tau}$ along with Factorization Lemma allowing to discard terms with extra powers of $\tau$ we obtain

$$
\begin{aligned}
h_{q,-\infty}^{\prime}\left(f^{\mathbf{i}}\right)= & -\lim _{\beta \rightarrow-\infty} \int_{0}^{1} d \tau(1-\beta \tau)^{-2} \int \frac{d^{2} u d^{2} v}{(2 \pi)^{2}} \exp i\left[v_{\alpha} u^{\alpha}\right] \exp i\left(\left[1-(1-\beta \tau)^{-1}\right] y_{\alpha} q^{\alpha}\right) \\
& \frac{\beta(1-\beta \tau)^{-1}}{\left[1-(1-\beta \tau)^{-1}\right]} \psi\left(-(q+u)\left[1-(1-\beta \tau)^{-1}\right],\left(v+y(1-\beta \tau)^{-1}\right), 0, \tau\right) .
\end{aligned}
$$

Hence by virtue of (8.11) and

$$
\int_{0}^{1} \mathrm{~d} \sigma(1-\sigma)^{n}=(n+1)^{-1}
$$

we obtain

$h_{q,-\infty}^{\prime}\left(f^{\mathbf{i}}\right)=-\int_{0}^{1} d \sigma(1-\sigma) \sigma^{-1} \int \frac{d^{2} u d^{2} v}{(2 \pi)^{2}} \exp i\left[v_{\alpha} u^{\alpha}+\sigma y_{\alpha} q^{\alpha}\right] \psi(-(q+u) \sigma, v+y(1-\sigma), 0,0)$.

Whence, using (6.2), one obtains (8.13). Note that $h_{q,-\infty}^{\prime}(f)$ acts as identity operator on the $z$-independent boundary term (6.4) associated with $\chi_{0}$ and by zero on $\chi_{2}$.

\section{Specific form degree relations}

So far we considered general relations like (6.25), (6.26) valid for $\theta$-forms of arbitrary degrees. For the practical analysis it is important to specify them further for the spaces $\mathcal{H}_{p}^{0+}$ and $\mathcal{H}_{p}^{+0}$ of $p$-forms of specific degrees. In this section we first derive star-product relations that greatly simplify computations, and then discuss the properties of limiting contracting homotopies acting on $\mathcal{H}_{1}$ and $\mathcal{H}_{2}$ resulting in the important Pre-Ultra-Locality Theorem.

\subsection{Star products}

In this section we compute star products for the spaces $\mathcal{H}_{0}$ and $\mathcal{H}_{1}$ up to the terms that belong to the ideal $\mathcal{I}$. The main reason for this is that such terms do not contribute to the field equations by Factorization Lemma (8.9) since $\mathcal{I}_{0} \subset \mathcal{H}_{0}^{+0}$ and $\triangle_{q,-\infty} \mathcal{I}_{1} \subset \mathcal{I}_{0}$ (9.25). In this section we focus on the more complicated star products of inner functions, omitting for brevity label i. Extension to boundary functions is evident.

\subsection{1 $\mathcal{H}_{0} * \mathcal{H}_{0}$}

Consider product (6.8) for $f_{0} * \tilde{f}_{0}$ where both $f_{0}$ and $\tilde{f}_{0}$ are zero-forms in $\theta$.

$$
\begin{aligned}
f_{0} * \tilde{f}_{0}= & \int_{0}^{1} d \tau_{1} \int_{0}^{1} d \tau_{2} \int \frac{d^{2} s d^{2} t}{(2 \pi)^{2}} \exp i\left[\tau_{1} \circ \tau_{2} z_{\alpha} y^{\alpha}+s_{\alpha} t^{\alpha}\right] \\
& \times \phi\left(\tau_{1}\left(\left(1-\tau_{2}\right) z-\tau_{2} y+s\right),\left(1-\tau_{1}\right)\left(\left(1-\tau_{2}\right) y-\tau_{2} z+s\right), \tau_{1}\right) \\
& \times \tilde{\phi}\left(\tau_{2}\left(\left(1-\tau_{1}\right) z+\tau_{1} y-t\right),\left(1-\tau_{2}\right)\left(\left(1-\tau_{1}\right) y+\tau_{1} z+t\right), \tau_{2}\right) .
\end{aligned}
$$


It is easy to see that

$$
f_{0}^{+0} * \tilde{f}_{0}^{+0} \simeq 0
$$

Indeed, since $f_{0}^{+0}$ has the form (6.1) with $\phi(u, w, \tau)=\tau \phi^{\prime}(u, w, \tau)$ carrying an additional factor of $\tau$, and similarly for $\tilde{f}_{0}^{+0}$, from (6.16) and (6.12) it follows that the product $f_{0}^{+0} * \tilde{f}_{0}^{+0}$ contains a factor dominated by $\tau_{1} \circ \tau_{2}\left(1-\tau_{1} \circ \tau_{2}\right)^{2}$ sending it to $\mathcal{I}$.

Computation of $f_{0}^{0+} * \tilde{f}_{0}^{+0}$ and $f_{0}^{+0} * \tilde{f}_{0}^{0+}$ gives using (6.13)

$$
\begin{aligned}
\mathcal{H}^{+0} \ni f_{0}^{0+} * \tilde{f}_{0}^{+0} \simeq & \int_{0}^{1} d \tau_{1}\left(1-\tau_{1}\right) \int_{0}^{1} d \tau_{2} \tau_{2} \int \frac{d^{2} s d^{2} t}{(2 \pi)^{2}} \exp i\left[\tau_{1} \circ \tau_{2} z_{\alpha} y^{\alpha}+s_{\alpha} t^{\alpha}\right] \\
& \times \phi\left(-\tau_{1} \tau_{2} y,\left(1-\tau_{1}\right)\left(\left(1-\tau_{2}\right) y-\tau_{2} z+s\right), \tau_{1}\right) \\
& \times \tilde{\phi}\left(\tau_{2}\left(\left(1-\tau_{1}\right) z+\tau_{1} y-t\right),\left(1-\tau_{1}\right)\left(1-\tau_{2}\right) y, \tau_{2}\right)
\end{aligned}
$$

Indeed, since $\tau$-kernel of $f_{0}^{0+} * \tilde{f}_{0}^{+0}(9.3)$ contains a factor of $\tau_{2}\left(1-\tau_{1}\right)$, from (6.16) and (6.13) it follows that terms proportional to $\tau_{1}$ or $1-\tau_{2}$ are dominated by $\left(\tau_{1} \circ \tau_{2}\left(1-\tau_{1} \circ \tau_{2}\right)\right)^{2}$ hence belonging to $\mathcal{I}$. Analogously,

$$
\begin{aligned}
\mathcal{H}^{+0} \ni f_{0}^{+0} * \tilde{f}_{0}^{0+} \simeq & \int_{0}^{1} d \tau_{1} \tau_{1} \int_{0}^{1} d \tau_{2}\left(1-\tau_{2}\right) \int \frac{d^{2} s d^{2} t}{(2 \pi)^{2}} \exp i\left[\tau_{1} \circ \tau_{2} z_{\alpha} y^{\alpha}+s_{\alpha} t^{\alpha}\right] \\
& \times \phi\left(\tau_{1}\left(\left(1-\tau_{2}\right) z-\tau_{2} y+s\right),\left(1-\tau_{1}\right)\left(1-\tau_{2}\right) y, \tau_{1}\right) \\
& \times \tilde{\phi}\left(\tau_{1} \tau_{2} y,\left(1-\tau_{2}\right)\left(\left(1-\tau_{1}\right) y+\tau_{1} z+t\right), \tau_{2}\right)
\end{aligned}
$$

In $f_{0}^{0+} * \tilde{f}_{0}^{0+}$, we can neglect the $s, t$ and $y$ dependence in the first arguments of $\phi$ and $\tilde{\phi}$. Indeed, since $\tau$-kernel of (9.5) contains a factor of $\left(1-\tau_{1}\right)\left(1-\tau_{2}\right)$, from (6.16) and (6.13) it follows that terms proportional to $\tau_{1}$ or $\tau_{2}$ are dominated by $\left(\tau_{1} \circ \tau_{2}\left(1-\tau_{1} \circ \tau_{2}\right)\right)^{2}$. As a result,

$$
\begin{aligned}
\mathcal{H}^{0+} \ni f_{0}^{0+} * \tilde{f}_{0}^{0+} \simeq & \int_{0}^{1} d \tau_{1}\left(1-\tau_{1}\right) \int_{0}^{1} d \tau_{2}\left(1-\tau_{2}\right) \int \frac{d^{2} s d^{2} t}{(2 \pi)^{2}} \exp i\left[\tau_{1} \circ \tau_{2} z_{\alpha} y^{\alpha}+s_{\alpha} t^{\alpha}\right] \\
& \times \phi\left(\tau_{1}\left(1-\tau_{2}\right) z,\left(1-\tau_{1}\right)\left(\left(1-\tau_{2}\right) y-\tau_{2} z+s\right), \tau_{1}\right) \\
& \times \tilde{\phi}\left(\tau_{2}\left(1-\tau_{1}\right) z,\left(1-\tau_{2}\right)\left(\left(1-\tau_{1}\right) y+\tau_{1} z+t\right), \tau_{2}\right)
\end{aligned}
$$

In particular, if $\phi(w, u, \tau)=\varphi(w, \tau)$, then $f_{0}^{0+} * \tilde{f}_{0}^{0+}$ contains no contractions

$$
\begin{aligned}
f_{0}^{0+} * \tilde{f}_{0}^{0+} \simeq & \int_{0}^{1} d \tau_{1} \int_{0}^{1} d \tau_{2} \exp i\left[\tau_{1} \circ \tau_{2} z_{\alpha} y^{\alpha}\right] \\
& \times \varphi\left(\tau_{1}\left(1-\tau_{2}\right) z, \tau_{1}\right) \tilde{\phi}\left(\tau_{2}\left(1-\tau_{1}\right) z,\left(1-\tau_{2}\right)\left(\left(1-\tau_{1}\right) y+\tau_{1} z\right), \tau_{2}\right) .
\end{aligned}
$$

Analogously, if $\tilde{\phi}(w, u, \tau)=\tilde{\varphi}(w, \tau)$

$$
\begin{aligned}
f_{0}^{0+} * \tilde{f}_{0}^{0+} \simeq & \int_{0}^{1} d \tau_{1} \int_{0}^{1} d \tau_{2} \exp i\left[\tau_{1} \circ \tau_{2} z_{\alpha} y^{\alpha}\right] \\
& \times \phi\left(\tau_{1}\left(1-\tau_{2}\right) z,\left(1-\tau_{1}\right)\left(\left(1-\tau_{2}\right) y-\tau_{2} z\right), \tau_{1}\right) \tilde{\varphi}\left(\tau_{2}\left(1-\tau_{1}\right) z, \tau_{2}\right)
\end{aligned}
$$

These formulae play the key role in the analysis of spin-locality of HS equations because they apply to the contributions involving the space-time one-form $W_{1}$ in either of the 
combinations $W_{1} * f$ or $f * W_{1}$ with $f$ being a zero-form in $\theta$. These terms contribute to the r.h.s. of the dynamical equations

$$
\mathrm{d} \omega+\omega * \omega+W_{1} * f+f * W_{1}+\ldots
$$

and

$$
\mathrm{d} C+[\omega, C]_{*}+\left[W_{1}, f\right]_{*}+\ldots
$$

implying ultra-locality of the $W_{1}$-depended terms provided that $f$ was spin-local in the lower orders. In particular, the contribution of $W_{1} * W_{1}$ to the field equations for $\omega$ turns out to be spin-local, which observation originally suggested spin-locality of the whole deformation in this sector.

\subsection{2 $\mathcal{H}_{0} * \mathcal{H}_{1}$ and $\mathcal{H}_{1} * \mathcal{H}_{0}$}

Let us consider product (6.8) for inner zero- and one-forms in $\theta, f_{0}$ and $f_{1}$, respectively. Being a zero-form, $f_{0}$ should contain a factor of $\frac{1-\tau_{1}}{\tau_{1}}$ in the pre-exponential. On the other hand, a one-form $f_{1}$ contains no prefactors. As a result, if the factor of $\tau_{1}^{-1}$ gets cancelled by one or another mechanism, taking into account the logarithmic factor (6.16), the resulting expression will belong to $\mathcal{I}$ and can be discarded. For instance, this allows us to discard the integration variable $s$ in the first argument of $f_{0}$ in (6.8) giving

$$
\begin{aligned}
f_{0} * f_{1} \simeq & \int_{0}^{1} d \tau_{1} \int_{0}^{1} d \tau_{2} \int \frac{d^{2} s d^{2} t}{(2 \pi)^{2}} \exp i\left[\tau_{1} \circ \tau_{2} z_{\alpha} y^{\alpha}+s_{\alpha} t^{\alpha}\right] \\
& \times \phi_{0}\left(\tau_{1}\left(\left(1-\tau_{2}\right) z-\tau_{2} y\right),\left(1-\tau_{1}\right)\left(\left(1-\tau_{2}\right) y-\tau_{2} z+s\right), \tau_{1} \theta, \tau_{1}\right) \\
& \times \phi_{1}\left(\tau_{2}\left(\left(1-\tau_{1}\right) z+\tau_{1} y-t\right),\left(1-\tau_{2}\right)\left(\left(1-\tau_{1}\right) y+\tau_{1} z+t\right), \tau_{2} \theta, \tau_{2}\right) .
\end{aligned}
$$

First of all we observe that if $f_{0} \in \mathcal{H}^{+0}$ containing an additional positive power of $\tau_{1}$ in $\tau$-kernel, then the whole result is in $\mathcal{I}$, i.e.

$$
f_{0}^{+0} * f_{1} \simeq 0 \text {. }
$$

Analogously,

$$
f_{1} * f_{0}^{+0} \simeq 0 .
$$

Thus, the products $f_{1} * f_{0}$ and $f_{0} * f_{1}$ can be nontrivial if $f_{0}=f_{0}^{0+} \in \mathcal{H}^{0+}$. Elementary analysis using (6.13) and (6.16) shows

$$
\begin{aligned}
\mathcal{H}_{1}^{+0} \ni f_{1}^{+0} * f_{0}^{0+} \simeq & \int_{0}^{1} d \tau_{1} \tau_{1} \int_{0}^{1} d \tau_{2}\left(1-\tau_{2}\right) \int \frac{d^{2} s d^{2} t}{(2 \pi)^{2}} \exp i\left[\tau_{1} \circ \tau_{2} z_{\alpha} y^{\alpha}+s_{\alpha} t^{\alpha}\right] \\
& \times \phi_{1}^{+0}\left(\tau_{1}\left(\left(1-\tau_{2}\right) z-\tau_{2} y+s\right),\left(1-\tau_{1}\right)\left(1-\tau_{2}\right) y, \tau_{1} \theta, \tau_{1}\right) \\
& \times \phi_{0}^{0+}\left(\tau_{2} \tau_{1} y,\left(1-\tau_{2}\right)\left(\left(1-\tau_{1}\right) y+\tau_{1} z+t\right), \tau_{2}\right) \\
\mathcal{H}_{1}^{0+} \ni f_{1}^{0+} * f_{0}^{0+} \simeq & \int_{0}^{1} d \tau_{1}\left(1-\tau_{1}\right) \int_{0}^{1} d \tau_{2}\left(1-\tau_{2}\right) \int \frac{d^{2} s d^{2} t}{(2 \pi)^{2}} \exp i\left[\tau_{1} \circ \tau_{2} z_{\alpha} y^{\alpha}+s_{\alpha} t^{\alpha}\right] \\
& \times \phi_{1}^{0+}\left(\tau_{1}\left(1-\tau_{2}\right) z,\left(1-\tau_{1}\right)\left(\left(1-\tau_{2}\right) y-\tau_{2} z+s\right), \tau_{1} \theta, \tau_{1}\right) \\
& \times \phi_{0}^{0+}\left(\tau_{2}\left(1-\tau_{1}\right) z,\left(1-\tau_{2}\right)\left(\left(1-\tau_{1}\right) y+\tau_{1} z+t\right), \tau_{2}\right)
\end{aligned}
$$




$$
\begin{aligned}
\mathcal{H}_{1}^{+0} \ni f_{0}^{0+} * f_{1}^{+0} \simeq & \int_{0}^{1} d \tau_{1}\left(1-\tau_{1}\right) \int_{0}^{1} d \tau_{2} \tau_{2} \int \frac{d^{2} s d^{2} t}{(2 \pi)^{2}} \exp i\left[\tau_{1} \circ \tau_{2} z_{\alpha} y^{\alpha}+s_{\alpha} t^{\alpha}\right] \\
& \times \phi_{0}^{0+}\left(-\tau_{1} \tau_{2} y,\left(1-\tau_{1}\right)\left(\left(1-\tau_{2}\right) y-\tau_{2} z+s\right), \tau_{1}\right) \\
& \times \phi_{1}^{+0}\left(\tau_{2}\left(\tau_{1} y+\left(1-\tau_{1}\right) z-t\right),\left(1-\tau_{2}\right)\left(1-\tau_{1}\right) y, \tau_{2}\right) \\
\mathcal{H}_{1}^{0+} \ni f_{0}^{0+} * f_{1}^{0+} \simeq & \int_{0}^{1} d \tau_{1}\left(1-\tau_{1}\right) \int_{0}^{1} d \tau_{2}\left(1-\tau_{2}\right) \int \frac{d^{2} s d^{2} t}{(2 \pi)^{2}} \exp i\left[\tau_{1} \circ \tau_{2} z_{\alpha} y^{\alpha}+s_{\alpha} t^{\alpha}\right] \\
& \times \phi_{0}^{0+}\left(\tau_{1}\left(1-\tau_{2}\right) z,\left(1-\tau_{1}\right)\left(\left(1-\tau_{2}\right) y-\tau_{2} z+s\right), \tau_{1}\right) \\
& \times \phi_{1}^{+0}\left(\tau_{2}\left(1-\tau_{1}\right) z,\left(1-\tau_{2}\right)\left(\left(1-\tau_{1}\right) y+\tau_{1} z+t\right), \tau_{2} \theta, \tau_{2}\right)
\end{aligned}
$$

An important consequence of these relations is that the star product of any one-form of the form $f_{1}=\theta^{\alpha} z_{\alpha} f$ with any zero-form keeps this form modulo terms in $\mathcal{H}_{1}^{+0}$.

\subsection{3 $\mathcal{H}_{1} * \mathcal{H}_{1}$}

To analyse star products of one-forms in $\theta^{\alpha}$ we should take into account that a two-form in $\theta^{\alpha}$ from $\mathcal{H}$ should contain an overall factor of $\frac{\tau}{1-\tau}$ in $\tau$-kernels. Since $f_{1}$ and $g_{1}$ had no overall factors in $\tau$-kernels, $f_{1} * g_{1}$ will be regular as well. Taking into account the contribution due to logarithm (6.16), this means that $f_{1} * g_{1}$ in fact contains two extra powers of $1-\tau_{1} \circ \tau_{2}$ in the $\tau$-kernel. Thus

$$
f_{1} * g_{1} \in \mathcal{H}_{2}^{0+} \quad \forall f_{1}, g_{1} \in \mathcal{H}_{1} .
$$

As a result, using (6.26), we obtain that

$$
f_{1}^{0+} * g_{1}^{+0} \simeq f_{1}^{+0} * g_{1}^{0+} \simeq 0 .
$$

The remaining two products are

$$
\begin{aligned}
\mathcal{H}_{2}^{0+} \ni f_{1}^{0+} * g_{1}^{0+} \simeq & \int_{0}^{1} d \tau_{1}\left(1-\tau_{1}\right) \int_{0}^{1} d \tau_{2}\left(1-\tau_{2}\right) \int \frac{d^{2} s d^{2} t}{(2 \pi)^{2}} \exp i\left[\tau_{1} \circ \tau_{2} z_{\alpha} y^{\alpha}+s_{\alpha} t^{\alpha}\right] \\
& \times \phi_{1 f}^{0+}\left(\tau_{1}\left(1-\tau_{2}\right) z,\left(1-\tau_{1}\right)\left(\left(1-\tau_{2}\right) y-\tau_{2} z+s\right), \tau_{1} \theta, \tau_{1}\right) \\
& \times \phi_{1 g}^{0+}\left(\tau_{2}\left(1-\tau_{1}\right) z,\left(1-\tau_{2}\right)\left(\left(1-\tau_{1}\right) y+\tau_{1} z+t\right), \tau_{2} \theta, \tau_{2}\right) \\
\mathcal{H}_{2}^{0+} \ni f_{1}^{+0} * g_{1}^{+0} \simeq & \int_{0}^{1} d \tau_{1} \tau_{1} \int_{0}^{1} d \tau_{2} \tau_{2} \int \frac{d^{2} s d^{2} t}{(2 \pi)^{2}} \exp i\left[\tau_{1} \circ \tau_{2} z_{\alpha} y^{\alpha}+s_{\alpha} t^{\alpha}\right] \\
& \times \phi_{1 f}^{+0}\left(\tau_{1}\left(\left(1-\tau_{2}\right) z-\tau_{2} y+s\right),-\tau_{2}\left(1-\tau_{1}\right) z, \tau_{1} \theta, \tau_{1}\right) \\
& \times \phi_{1 g}^{+0}\left(\tau_{2}\left(\left(1-\tau_{1}\right) z+\tau_{1} y-t\right), \tau_{1}\left(1-\tau_{2}\right) z, \tau_{2} \theta, \tau_{2}\right)
\end{aligned}
$$

This completes the list of star products between inner zero- and one-forms in $\theta$. Star products $\mathcal{H}_{0} * \mathcal{H}_{2}$ and $\mathcal{H}_{2} * \mathcal{H}_{0}$ follow from $\mathcal{H}_{0} * \mathcal{H}_{0}$ with the help of $\gamma$-maps of section 6.2.1.

\subsection{Limiting contracting homotopy}

Contracting homotopies with general parameters $-\infty<\beta<1$ do not leave the spaces $\mathcal{H}^{\nu \mu}$ invariant. A distinguishing feature of the $\beta \rightarrow-\infty$ limiting homotopy is that, as shown in this section, it exhibits special properties when acting on the spaces $\mathcal{H}_{p}^{\nu \mu}$, that underly spin-locality of HS interactions and allow us to formulate a sufficient condition for the limit $\beta \rightarrow-\infty$ be well defined. 


\subsubsection{Contracting homotopy of $\mathcal{H}_{1}^{\nu \mu}$}

For inner $f_{1} \in \mathcal{H}_{1}^{\nu \mu}$ formula (8.2) gives

$$
\begin{aligned}
\triangle_{q,-\infty}^{\prime}\left(f_{1}\right)= & \frac{1}{(2 \pi)^{2}} \int d^{2} v d^{2} u \int d_{+}^{3} \tau \delta\left(1-\sum_{i=1}^{3} \tau_{i}\right) \exp i\left[v_{\alpha} u^{\alpha}+\tau_{1} z_{\alpha} y^{\alpha}-\tau_{2} q_{\beta} y^{\beta}\right] \\
& \times\left(\left(z^{\beta}+q^{\beta}\right)+\frac{\tau_{3}}{\tau_{1}}\left(u^{\beta}+q^{\beta}\right)\right) \frac{\partial}{\partial \theta^{\beta}} \psi\left(\tau_{1} z-\frac{\tau_{2} \tau_{3}}{\tau_{1}+\tau_{3}} u-\tau_{2} q, v+\tau_{3} y, \theta, \frac{\tau_{1}}{\tau_{1}+\tau_{3}}\right) .
\end{aligned}
$$

Generally, this formula may have logarithmic divergency due to $\tau_{1}$ integration of the term $\frac{\tau_{3}}{\tau_{1}}\left(u^{\beta}+q^{\beta}\right)$. This does not happen however at least in the following two cases.

First, if $f_{1}^{+0} \in \mathcal{H}_{1}^{+0}$ then $\psi$ contains an additional factor of $\frac{\tau_{1}}{\tau_{1}+\tau_{3}}$ that cancels the divergency. Since, by virtue of $(8.8), \tau_{3}$ integration brings a factor of $\tau_{1}\left(1-\tau_{1}\right)$ we find that

$$
\triangle_{q,-\infty}^{\prime} \mathcal{H}_{1}^{+0} \subset \mathcal{H}_{0}^{+0} .
$$

Second, if $f_{1}^{0+} \in \mathcal{H}_{1}^{0+}$, then $\psi$ contains an additional factor of

$$
1-\frac{\tau_{1}}{\tau_{1}+\tau_{3}}=\frac{\tau_{3}}{\tau_{1}+\tau_{3}} .
$$

Since, up to non-essential logarithmic terms,

$$
\int_{0}^{1-\tau_{1}} \frac{\tau_{3}}{\tau_{1}+\tau_{3}} \sim 2\left(1-\tau_{1}\right)^{2}+O\left(\left(1-\tau_{1}\right)^{3}\right),
$$

the resulting expression at r.h.s. of $(9.21)$ behaves as $\left(1-\tau_{1}\right)^{2}$ at $\tau_{1} \rightarrow 1$ as it has to in $\mathcal{H}_{0}^{0+}$.

From here and (9.22) follows an important fact that

$$
\triangle_{q,-\infty}^{\prime} \mathcal{I}_{1} \subset \mathcal{I}_{0}
$$

allowing to discard the contribution of $\mathcal{I}_{1}$ to $\mathcal{H}_{1}^{+0}$ in (9.22). As a consequence we obtain

$\omega$-Lemma: Elements of $\mathcal{I}$ can be discarded in all terms containing space-time one-forms $\omega$.

Indeed, such terms can never contribute to the sector of two-forms in $\theta^{\alpha}$ via star product, allowing us to use (9.25) along with Factorization Lemma (8.9) implying that $\mathcal{I} \subset \mathcal{H}^{+0}$ does not contribute under the cohomology projector.

However, to belong to $\mathcal{H}_{0}^{0+}, \Delta_{q,-\infty}^{\prime}\left(f_{1}\right)(9.21)$ should have a fictitious pole in $\tau_{1}$ obeying (6.5) which is true if the whole expression consists of terms proportional to $z^{\alpha}$ or carrying an additional power of $\tau$ (the latter terms belong to $\mathcal{H}^{+0}$ ). As we show now, this is the case if $f_{1}^{0+} \sim z_{\alpha} \theta^{\alpha}$.

\subsubsection{Space $\widetilde{\mathcal{H}}_{1}^{0+}$}

Let $\widetilde{\mathcal{H}}_{1}^{0+}$ be the subspace of $\mathcal{H}_{1}^{0+}$ that consists of the one-forms proportional of $z_{\alpha} \theta^{\alpha}$. In other words, consider $\tilde{f}_{1}$ with $\psi(6.2)$ of the form

$$
\psi(w, u, \theta, \tau)=w_{\alpha} \theta^{\alpha} \tilde{\psi}(w, u, \tau) .
$$


Then formula (9.21) gives

$$
\begin{aligned}
\triangle_{q,-\infty}^{\prime}\left(\tilde{f}_{1}\right)= & \frac{1}{(2 \pi)^{2}} \int d^{2} v d^{2} u \int d_{+}^{3} \tau \delta\left(1-\sum_{i=1}^{3} \tau_{i}\right) \exp i\left[v_{\alpha} u^{\alpha}+\tau_{1} z_{\alpha} y^{\alpha}-\tau_{2} q_{\beta} y^{\beta}\right] \\
& \times z_{\beta}\left(\frac{\tau_{3}}{\tau_{1}+\tau_{3}} u^{\beta}+q^{\beta}\right) \tilde{\psi}\left(\tau_{1} z-\frac{\tau_{2} \tau_{3}}{\tau_{1}+\tau_{3}} u-\tau_{2} q, v+\tau_{3} y, \frac{\tau_{1}}{\tau_{1}+\tau_{3}}\right),
\end{aligned}
$$

whence, by virtue of $(9.24)$

$$
\triangle_{q,-\infty}^{\prime} \widetilde{\mathcal{H}}_{1}^{0+} \subset \mathcal{H}_{0}^{0+} .
$$

On the other hand, $\triangle_{q,-\infty}^{\prime} \mathcal{H}_{1}^{0+}$ for generic elements of $\mathcal{H}_{1}^{0+}$ may be away from $\mathcal{H}_{0}^{0+}$ giving rise to divergent expressions. (Recall that, since for any finite $\beta<1$ contracting homotopies $\triangle_{q, \beta}$ give finite results, this would just mean that the limit $\beta \rightarrow-\infty$ is illdefined.) In the next section we formulate a sufficient condition guaranteeing that this does not happen. Then in section 11 it will be shown that these conditions are indeed fulfilled at least to the order $\omega^{2} C^{2}$.

The following comment is now in order. Consider a one-form $f_{1}$ containing an overall factor $z_{\alpha} \theta^{\alpha}$

$$
f_{1}(z, y, \theta)=z_{\alpha} \theta^{\alpha} \int_{0}^{1} d \tau \tau \psi(\tau z,(1-\tau) y) \exp i \tau z_{\alpha} y^{\alpha} .
$$

Using decomposition (6.27) consider its $f_{1}^{0+}$ part (6.29)

$$
f_{1}^{0+}(z, y, \theta)=z_{\alpha} \theta^{\alpha} \int_{0}^{1} d \tau \tau(1-\tau) \psi(\tau z,(1-\tau) y) \exp i \tau z_{\alpha} y^{\alpha} .
$$

The remarkable fact is that

$$
\mathrm{d}_{z} f_{1}^{0+}(z, y, \theta) \simeq 0
$$

Indeed,

$$
\begin{aligned}
\mathrm{d}_{z} f^{0+}(z, y)= & \int_{0}^{1} d \tau \tau(1-\tau) \exp i\left(\tau z_{\alpha} y^{\alpha}\right)\left(\theta_{\gamma} \theta^{\gamma}-i \tau \theta^{\gamma} z_{\gamma} \theta_{\beta} y^{\beta}-\theta^{\gamma} z_{\gamma} \theta^{\alpha} \frac{\partial}{\partial z^{\alpha}}\right) \psi(\tau z,(1-\tau) y) \\
= & \theta_{\gamma} \theta^{\gamma} \int_{0}^{1} d \tau_{1} d \tau_{2} \theta\left(\tau_{1}\right) \theta\left(\tau_{2}\right) \delta\left(1-\tau_{1}-\tau_{2}\right) \\
& \times \tau_{1} \tau_{2}\left(1+\frac{1}{2} \tau_{1} \frac{\partial}{\partial \tau_{1}}\right)\left[\psi\left(\tau_{1} z, \tau_{2} y\right) \exp i\left(\tau_{1} z_{\alpha} y^{\alpha}\right)\right] \\
= & \frac{1}{2} \theta_{\gamma} \theta^{\gamma} \int_{0}^{1} d \tau_{1} d \tau_{2} \theta\left(\tau_{1}\right) \theta\left(\tau_{2}\right) \delta\left(1-\tau_{1}-\tau_{2}\right) \tau_{1}^{2} \frac{\partial}{\partial \tau_{2}}\left(\tau_{2} \psi\left(\tau_{1} z, \tau_{2} y\right)\right) \exp i\left(\tau_{1} z_{\alpha} y^{\alpha}\right) .
\end{aligned}
$$

The last term belongs to $\mathcal{I}$ because it contains an additional factor of $\tau_{1} \tau_{2}$ compared to the normally assigned to a two-form in $\mathcal{H}_{2}$ by (6.1), (6.3).

Relation (9.31) has a consequence that any element of $\widetilde{\mathcal{H}}_{1}{ }^{0+}$ is weakly $\mathrm{d}_{z}$-closed. This is because any modification of the $\tau$-dependence in formula (9.29) within the class $\mathcal{H}_{1}^{0+}$ that leaves the leading $\tau$-dependence intact will only contribute to $\mathcal{I}$. 
It is useful to introduce the space $\widetilde{\mathcal{H}}_{1}$

$$
\widetilde{\mathcal{H}}_{1}:=\operatorname{Span}\left(\widetilde{\mathcal{H}}_{1}{ }^{0+}, \mathcal{H}_{1}^{+0}\right)
$$

Relation (9.31) implies that

$$
\mathrm{d}_{z} \widetilde{\mathcal{H}}_{1} \subset \mathcal{H}_{2}^{+0} .
$$

$\widetilde{\mathcal{H}}_{1}$ is just the space that leads to the finite result under the action of the limiting contracting homotopy, i.e.

$$
\triangle_{q,-\infty}^{\prime} \widetilde{\mathcal{H}}_{1} \subset \mathcal{H}_{0}
$$

From (9.13)-(9.16) it follows that the result of star product of any element of $\widetilde{\mathcal{H}}_{1}$ and any element of $\mathcal{H}_{0}$ is either proportional to $z^{\alpha} \theta_{\alpha}$ or belongs to $\mathcal{H}_{1}^{+0}$. Hence it holds remarkable

$\widetilde{\mathcal{H}}_{1}$ Closure Lemma

$$
\widetilde{\mathcal{H}}_{1} * \mathcal{H}_{0} \subset \widetilde{\mathcal{H}}_{1}, \quad \mathcal{H}_{0} * \widetilde{\mathcal{H}}_{1} \subset \widetilde{\mathcal{H}}_{1}
$$

$\widetilde{\mathcal{H}}_{1}$ Closure Lemma has an important consequence that star product of $S \in \widetilde{\mathcal{H}}_{1}$ with HS fields $W$ or $B$ still belongs to $\widetilde{\mathcal{H}}_{1}$.

\subsubsection{Contracting homotopy of $\mathcal{H}_{2}^{\nu \mu}$}

The contracting homotopy $\triangle_{q,-\infty}^{\prime}$ does not leave the spaces $\mathcal{H}^{\nu \mu}$ invariant. Let us show that $\triangle_{q,-\infty}^{\prime} \mathcal{H}_{2}^{+0} \notin \mathcal{H}_{1}^{+0}$. Consider $f_{2}^{+0}$ with $\psi^{+0}$ of the form

$$
\psi^{+0}(w, u, \theta, \tau)=\theta^{\alpha} \theta_{\alpha} \tau \tilde{\psi}^{+0}(w, u, \tau) .
$$

Then, by (8.2),

$$
\begin{aligned}
\triangle_{q,-\infty}^{\prime}\left(f_{2}^{+0}\right)= & 2 \frac{1}{(2 \pi)^{2}} \int d^{2} v d^{2} u \int d_{+}^{3} \tau \delta\left(1-\sum_{i=1}^{3} \tau_{i}\right) \exp i\left[v_{\alpha} u^{\alpha}+\tau_{1} z_{\alpha} y^{\alpha}-\tau_{2} q_{\beta} y^{\beta}\right] \\
& \times \frac{\tau_{1}}{\tau_{1}+\tau_{3}}\left(\frac{\tau_{1}}{\tau_{3}}\left(z^{\beta}+q^{\beta}\right)+\left(u^{\beta}+q^{\beta}\right)\right) \\
& \times \theta_{\beta} \tilde{\psi}^{+0}\left(\tau_{1} z-\frac{\tau_{2} \tau_{3}}{\tau_{1}+\tau_{3}} u-\tau_{2} q, v+\tau_{3} y, \frac{\tau_{1}}{\tau_{1}+\tau_{3}}\right) .
\end{aligned}
$$

Decomposing $f_{2}^{+0}=f_{2}^{\mathbf{i}+0}+f_{2}^{\mathbf{b}+0}$, firstly we note that this happens already to the boundary term. Indeed, any boundary term $f_{2}^{\mathbf{b}}$ can be rewritten as $f_{2}^{\mathbf{b}}=F(y) * \gamma$ for some $F(y)$. Using the star-exchange formulae along with the identity $\triangle_{q, \beta}^{\prime} \gamma=\Delta_{q, 0} \gamma[1]$, we have

$$
\triangle_{q,-\infty}^{\prime} f_{2}^{\mathbf{b}}=\triangle_{q, 0} f_{2}^{\mathbf{b}} .
$$

One can see that $\triangle_{q,-\infty}^{\prime} f_{2}^{\mathbf{b}+0}$ belongs to full $\mathcal{H}_{1}$, contributing, in particular to $\mathcal{H}_{1}^{0+}$. It is by this mechanism the non-trivial first-order contribution to HS field equations comes from the term $\gamma * C \in \mathcal{H}_{2}^{+0}$ on the r.h.s. of (4.6). Moreover, from (9.40) along with (7.21) it follows that, for a zero shift $q=0$, the result is proportional to $z_{\alpha} \theta^{\alpha}$, i.e.

$$
\triangle_{0,-\infty} f_{2}^{\mathbf{b}+0} \in \widetilde{\mathcal{H}}_{1} .
$$


Let us now consider the contribution $\Delta_{q,-\infty}^{\prime} f_{2}^{\mathbf{i}+0}$ of the inner part. To see what happens, first of all note that the pole in $\tau_{3}$ is fictitious because, in agreement with (6.3), $\tilde{\psi}_{1}^{+0}(w, r, \tau)$ must be linear in the second argument (up to possible $y$-independent terms from $\mathcal{I}$ that carry an additional power of $\left.\frac{\tau_{3}}{\tau_{1}+\tau_{3}}\right)$. Indeed, both $\tau_{3} y$ and $v_{\alpha}$ being equivalent to $i \frac{\partial}{\partial u^{\alpha}}$ then bring a factor of $\tau_{3}$ that cancels $\tau_{3}^{-1}$ in the pre-exponential. More in detail, setting

$$
\tilde{\psi}(w, r, \tau)=r_{\alpha} \tilde{\psi}^{\alpha}(w, r, \tau)
$$

and changing integration variables $u \rightarrow\left(\tau_{1}+\tau_{3}\right) \tau_{3}^{-1} u, v \rightarrow \tau_{3}\left(\tau_{1}+\tau_{3}\right)^{-1} v$ we obtain from (9.21)

$$
\begin{aligned}
\tilde{f}_{1}:=\triangle_{q,-\infty}^{\prime}\left(f_{2}^{\mathbf{i}+0}\right)= & \frac{2}{(2 \pi)^{2}} \int d^{2} v d^{2} u \int d_{+}^{3} \tau \delta\left(1-\sum_{i=1}^{3} \tau_{i}\right) \exp i\left[v_{\alpha} u^{\alpha}+\tau_{1} z_{\alpha} y^{\alpha}-\tau_{2} q_{\beta} y^{\beta}\right] \\
& \times \frac{\tau_{1}}{\tau_{1}+\tau_{3}} \theta_{\beta}\left(\tau_{1} z^{\beta}+\left(\tau_{1}+\tau_{3}\right)(u+q)^{\beta}\right)\left(\frac{v_{\alpha}}{\tau_{1}+\tau_{3}}+y_{\alpha}\right) \\
& \times \tilde{\psi}^{+0 \alpha}\left(\tau_{1} z-\tau_{2}(u+q), \frac{\tau_{3}}{\tau_{1}+\tau_{3}} v+\tau_{3} y, \frac{\tau_{1}}{\tau_{1}+\tau_{3}}\right)
\end{aligned}
$$

It is not hard to see that most of the terms in this expression belong to $\mathcal{H}_{1}^{+0}$ except for one. Namely, $v_{\alpha}$ in front of $\psi$ can be replaced by $i \frac{\partial}{\partial u^{\alpha}}$. The important contribution is from the differentiation of the first argument of $\tilde{\psi}^{+0 \alpha}$. Neglecting terms from $\mathcal{H}_{1}^{+0}$ this gives

$$
\begin{aligned}
& -2 i \frac{1}{(2 \pi)^{2}} \int d^{2} v d^{2} u \int d_{+}^{3} \tau \delta\left(1-\sum_{i=1}^{3} \tau_{i}\right) \exp i\left[v_{\alpha} u^{\alpha}+\tau_{1} z_{\alpha} y^{\alpha}-\tau_{2} q_{\beta} y^{\beta}\right] \\
& \times \frac{\tau_{1} \tau_{2}}{\left(\tau_{1}+\tau_{3}\right)^{2}}\left(\tau_{1} z^{\beta}+\left(\tau_{1}+\tau_{3}\right)(u+q)^{\beta}\right) \\
& \times \theta_{\beta} \partial_{1 \alpha} \tilde{\psi}^{+0 \alpha}\left(\tau_{1} z-\tau_{2}(u+q), \frac{\tau_{3}}{\tau_{1}+\tau_{3}} v+\tau_{3} y, \frac{\tau_{1}}{\tau_{1}+\tau_{3}}\right) .
\end{aligned}
$$

By virtue of (8.3)-(8.5), all $z$-independent terms in the pre-exponential belong to $\mathcal{H}_{1}^{+0}$. However, by virtue of (8.4), the $z$-dependent term contributes to $\mathcal{H}_{1}^{0+}$ giving

$$
\begin{aligned}
& -2 i \frac{1}{(2 \pi)^{2}} \int d^{2} v d^{2} u \int d_{+}^{3} \tau \delta\left(1-\sum_{i=1}^{3} \tau_{i}\right) \exp i\left[v_{\alpha} u^{\alpha}+\tau_{1} z_{\alpha} y^{\alpha}-\tau_{2} q_{\beta} y^{\beta}\right] \\
& \times \frac{\tau_{1}^{2} \tau_{2}}{\left(\tau_{1}+\tau_{3}\right)^{2}} z^{\beta} \theta_{\beta} \partial_{1 \alpha} \tilde{\psi}^{+0 \alpha}\left(\tau_{1} z-\tau_{2}(q+u), \frac{\tau_{3}}{\tau_{1}+\tau_{3}} v+\tau_{3} y, \frac{\tau_{1}}{\tau_{1}+\tau_{3}}\right) .
\end{aligned}
$$

Finally, taking into account that $\tau_{2}=1-\tau_{1}-\tau_{3}$ due to the delta-function $\delta\left(1-\sum_{i=1}^{3} \tau_{i}\right)$ and that any additional factor of $\tau_{1}+\tau_{3}$ or $\tau_{3}$ effectively increases the power of $\tau_{1}$ hence sending the result to $\mathcal{H}_{1}^{+0}$, we can replace $\tau_{2}$ by one and neglect the $y$-dependent terms in the argument of $\tilde{\psi}^{+0 \alpha}$, that carry an additional factor of $\tau_{3}$, arriving at the final result 


$$
\begin{aligned}
& \tilde{f}_{1}^{0+} \in \tilde{\mathcal{H}}_{1}^{0+} \text { with } \\
& \begin{aligned}
\tilde{f}_{1}^{0+}:=\left.\tilde{f}_{1}\right|_{\bmod \mathcal{H}_{1}^{+0}}= & -\frac{2 i}{(2 \pi)^{2}} \int d^{2} v d^{2} u \int d_{+}^{3} \tau \delta\left(1-\sum_{i=1}^{3} \tau_{i}\right) \frac{\tau_{1}^{2}}{\left(\tau_{1}+\tau_{3}\right)^{2}} z^{\beta} \theta_{\beta} \\
& \times \exp i\left[v_{\alpha} u^{\alpha}+\tau_{1} z_{\alpha} y^{\alpha}-\left(1-\tau_{1}\right) q_{\beta} y^{\beta}\right] \\
& \times \partial_{1 \alpha} \tilde{\psi}^{+0 \alpha}\left(\tau_{1} z-u-q, \frac{\tau_{3}}{\tau_{1}+\tau_{3}} v, \frac{\tau_{1}}{\tau_{1}+\tau_{3}}\right) .
\end{aligned}
\end{aligned}
$$

Note that for $r$-independent $\tilde{\psi}$ in (9.42), using that $\triangle_{q,-\beta}^{\prime}\left(\tilde{f}_{2}^{\mathbf{i}+0}\right)$ is $\beta$-independent, one easily obtains that $\tilde{f}_{1}^{0+} \in \widetilde{\mathcal{H}}_{1}{ }^{0+}$ as well, where

$$
\tilde{f}_{1}^{0+}:=\left.\tilde{f}_{1}\right|_{\bmod \mathcal{H}^{+0}}=2 \int d \tau \tau(1-\tau) z^{\beta} \theta_{\beta} \exp i\left[\tau z_{\alpha} y^{\alpha}-(1-\tau) q_{\beta} y^{\beta}\right] \tilde{\psi}^{+0}(\tau z-q, 0, \tau) .
$$

Taking into account the definition $(9.33)$ of $\widetilde{\mathcal{H}}_{1}$, we arrive at

$\mathcal{H}_{2}^{+0}$ Homotopy Lemma:

$$
\triangle_{q,-\infty}^{\prime} \mathcal{H}_{2}^{\mathbf{i}+0} \subset \widetilde{\mathcal{H}}_{1} .
$$

Note that setting $q=0$ in (9.49) we achieve that $\mathcal{H}_{2}^{+0}$ Homotopy Lemma (9.49) holds for the whole $\mathcal{H}_{2}^{+0}$ including the boundary elements associated with $\chi_{2}$ (6.2).

$\mathcal{H}_{2}^{+0}$ Homotopy Lemma is of great importance for the analysis of HS vertices. A representative of $\widetilde{\mathcal{H}}_{1}^{0+}$ for inner elements of $\mathcal{H}_{2}^{+0}$ can be chosen in the form of (9.46) or (9.48).

The following comment is now in order. If $\triangle_{q, \beta} \mathcal{H}_{2}^{+0}$ is evaluated at finite $\beta=-\varepsilon^{-1}$ the result may have the form

$$
\triangle_{q, \beta} \mathcal{H}_{2}^{+0} \in \widetilde{\mathcal{H}}_{1}+\varepsilon \mathcal{H}_{1}^{0+}
$$

i.e. the contribution to $\mathcal{H}^{0+}$ may be non-zero, being suppressed by the factor of $\varepsilon$. If, following the strategy of [1], one would keep $\beta$ finite till arriving to the final result containing further action of $\triangle_{q, \beta}$ and $h_{q, \beta}$ taking the limit $\beta \rightarrow-\infty$ in the very end, this may lead to finite but different result since, being in general singular, $\triangle_{q, \beta} \mathcal{H}^{0+}$ may develop the terms containing a factor of $\varepsilon^{-1}$ that can cancel $\varepsilon$ in (9.50). Nevertheless, the final result will still be ultra-local because the form of the exponentials (7.35) remains unaffected by this procedure. Each of these limiting procedures is properly defined. Generally, one can consider three limiting parameters $\beta_{2}$ on two-forms, $\beta_{1}$ on one-forms and $\beta_{0}$ on zero-forms (in the cohomology projector $h_{q, \beta_{0}}$ ) with $\beta_{i}=\alpha_{i} \beta$ allowing various ratios of $\alpha_{i}$ including $\frac{\alpha_{i}}{\alpha_{j}} \rightarrow 0$ at $i>j$ implying that the limit $\beta \rightarrow-\infty$ is taken at every step as in this paper. The procedure of [1] assumes $\alpha_{2}=\alpha_{1}=\alpha_{0}=1$. The results of application of different limiting prescriptions may differ at most by ultra-local field redefinitions.

\section{Pre-ultra-locality and ultra-locality}

The results of section 9.2 have a number of important consequences allowing to prove ultralocality of the vertices $\Upsilon_{2}^{\omega}\left(\omega^{2}, C^{2}\right)$ (for the definition of ultra-locality see sections 3.4, 3.6) in equations (3.3) on space-time one-forms $\omega$. 
A remarkable property of formula (9.46) is that $f_{1}^{0+}$ is free from $y$-dependence in the arguments of $C$ if $q$ is independent of $C$-derivatives $p_{j}$, in particular at $q=0$. Elements of $\mathcal{H}$ such that arguments of zero-forms $C$ are independent of $y$, will be called pre-ultra-local. Note that for elements bilinear in the zero-forms $C$, that respect the PLT conditions, preultra-locality implies ultra-locality by virtue of (7.37) from which it follows that $P_{i j}=0$ once $B_{i}=0$ and $i=1,2$.

A subspace of $\mathcal{H}^{\nu \mu}$ that consists of pre-ultra-local forms will be denoted $\mathcal{P}^{\nu \mu}$. The space $\mathcal{U}^{\nu \mu} \subset \mathcal{P}^{\nu \mu}$ of ultra-local forms consists of elements with at most a finite number of contractions between either holomorphic or anti-holomorphic arguments of the zero-forms $C$.

Now we consider properties of these two spaces separately.

\subsection{Pre-ultra-locality}

\subsubsection{Pre-ultra-local spaces}

From formulae (9.6), (9.7) it follows that the space $\mathcal{P}_{0}^{0+} \in \mathcal{H}_{0}^{0+}$ of pre-ultra-local zero-forms in $\theta$ is closed under the star product modulo terms in ideal $\mathcal{I}$

$$
\mathcal{P}_{0}^{0+} * \mathcal{P}_{0}^{0+} \subset \operatorname{Span}\left(\mathcal{P}_{0}^{0+}, \mathcal{I}\right) \text {. }
$$

Indeed, by definition of pre-ultra-locality all additional contractions in (9.6), (9.7) among the $y$-dependent terms will not affect arguments of zero-forms $C$ free from the $y$-dependence. From relations (9.2)-(9.4) it also follows that the space

$$
\mathcal{P}_{0}:=\operatorname{Span}\left(\mathcal{P}_{0}^{0+}, \mathcal{H}_{0}^{+0}\right)
$$

forms a subspace of $\mathcal{H}_{0}$

$$
\mathcal{P}_{0} * \mathcal{P}_{0} \subset \mathcal{P}_{0} \subset \mathcal{H}_{0}
$$

Clearly,

$$
\mathcal{P}_{0}^{0+} * \mathcal{P}_{0} \subset \mathcal{P}_{0}, \quad \mathcal{P}_{0} * \mathcal{P}_{0}^{0+} \subset \mathcal{P}_{0}
$$

Analogously, the space of pre-ultra-local one-forms $\mathcal{P}_{1}^{0+} \subset \mathcal{H}_{1}^{0+}$ and

$$
\mathcal{P}_{1}:=\operatorname{Span}\left(\mathcal{P}_{1}^{0+}, \mathcal{H}_{1}^{+0}\right)
$$

form $\mathcal{P}_{0}^{0+}$ - and $\mathcal{P}_{0}$ - bi-modules up to elements in $\mathcal{I}$

$$
\begin{array}{rlrl}
\mathcal{P}_{0}^{0+} * \mathcal{P}_{1}^{0+} \subset \operatorname{Span}\left(\mathcal{P}_{1}^{0+}, \mathcal{I}\right), & & \mathcal{P}_{1}^{0+} * \mathcal{P}_{0}^{0+} \subset \operatorname{Span}\left(\mathcal{P}_{1}^{0+}, \mathcal{I}\right), \\
& \mathcal{P}_{0} * \mathcal{P}_{1} \subset \mathcal{P}_{1}, & & \mathcal{P}_{1} * \mathcal{P}_{0} \subset \mathcal{P}_{1} .
\end{array}
$$

Introducing the space $\widetilde{\mathcal{P}}_{1}{ }^{0+} \subset \widetilde{\mathcal{H}}_{1}{ }^{0+}$ as the pre-ultra-local subspace of the space $\widetilde{\mathcal{H}}_{1}{ }^{0+}$ of section 9.2.2 we define a space

$$
\widetilde{\mathcal{P}}_{1}:=\operatorname{Span}\left(\widetilde{\mathcal{P}}_{1}{ }^{0+}, \mathcal{H}_{1}^{+0}\right)
$$

and using again product formulae (9.14) and (9.16) obtain

$$
\begin{gathered}
\mathcal{P}_{0}^{0+} * \widetilde{\mathcal{P}}_{1}^{0+} \subset \operatorname{Span}\left(\widetilde{\mathcal{P}}_{1}{ }^{0+}, \mathcal{I}\right) \subset \widetilde{\mathcal{P}}_{1}, \quad \widetilde{\mathcal{P}}_{1}{ }^{0+} * \mathcal{P}_{0}^{0+} \subset \operatorname{Span}\left(\widetilde{\mathcal{P}}_{1}^{0+}, \mathcal{I}\right) \subset \widetilde{\mathcal{P}}_{1}, \\
\mathcal{P}_{0} * \widetilde{\mathcal{P}}_{1} \subset \widetilde{\mathcal{P}}_{1}, \quad \widetilde{\mathcal{P}}_{1} * \mathcal{P}_{0} \subset \widetilde{\mathcal{P}}_{1}
\end{gathered}
$$




\subsubsection{Consequences}

Formula (9.46) along with (10.8) implies the following

Pre-Ultra-Locality Theorem:

$$
\triangle_{0,-\infty}^{\prime} \mathcal{H}_{2}^{+0} \subset \widetilde{\mathcal{P}}_{1}
$$

from which it follows that if the r.h.s. of equations for $S$ is in $\mathcal{H}_{2}^{+0}$ then, at this order, $S \in \widetilde{\mathcal{P}}_{1}$.

As a simple consequence of (8.13) the arguments of zero-forms $C$ in $h_{0,-\infty}\left(\mathcal{P}_{0}^{0+}\right)$ are $y$-independent. (More generally this is true for $h_{q,-\infty}\left(\mathcal{P}_{0}^{0+}\right)$ with $q$ not acting on the arguments of $C$.) Hence, using Factorization Lemma (8.9), one has

$$
h_{0,-\infty}\left(\mathcal{P}_{0}\right) \subset \mathcal{P}_{0}^{0+} .
$$

Note that the r.h.s. here only contains terms with boundary $\tau$-kernels (6.4).

Analogously, from (9.27) it follows that the arguments of zero-forms $C$ in $\triangle_{q,-\infty}\left(\mathcal{P}_{1}^{0+}\right)$ with $C$-derivative-independent $q$ are $y$-independent. Hence, taking into account (9.28) and definition (10.8), we obtain

$$
\triangle_{0,-\infty}\left(\widetilde{\mathcal{P}}_{1}\right) \subset \mathcal{P}_{0}
$$

As a result, by virtue of (10.3), (10.10) and (10.12) along with Factorization Lemma,

$$
\begin{array}{rlrl}
h_{0,-\infty}\left(\triangle_{0,-\infty}\left(\mathcal{P}_{0} * \widetilde{\mathcal{P}}_{1}\right) * \mathcal{P}_{0}\right) & \subset \mathcal{P}_{0}^{0+}, & h_{0,-\infty}\left(\Delta_{0,-\infty}\left(\widetilde{\mathcal{P}}_{1} * \mathcal{P}_{0}\right) * \mathcal{P}_{0}\right) \subset \mathcal{P}_{0}^{0+}, \\
h_{0,-\infty}\left(\mathcal{P}_{0} * \triangle_{0,-\infty}\left(\mathcal{P}_{0} * \widetilde{\mathcal{P}}_{1}\right)\right) \subset \mathcal{P}_{0}^{0+}, & h_{0,-\infty}\left(\mathcal{P}_{0} * \triangle_{0,-\infty}\left(\widetilde{\mathcal{P}}_{1} * \mathcal{P}_{0}\right)\right) \subset \mathcal{P}_{0}^{0+}
\end{array}
$$

In particular, from here it follows by virtue of Pre-Ultra-Locality Theorem (10.11)

$$
h_{0,-\infty}\left(\triangle_{0,-\infty}\left(\triangle_{0,-\infty}\left(\mathcal{H}_{2}^{+0}\right) * \mathcal{P}_{0}\right) * \mathcal{P}_{0}\right) \subset \mathcal{P}_{0}^{0+}, \quad \text { etc. }
$$

For expressions bilinear in the zero-forms $C$ pre-ultra-locality implies ultra-locality by virtue of PLT. As explained in sections 11 and 12, this proves that the vertices $\Upsilon_{2}^{\omega}\left(\omega^{2}, C^{2}\right)$ in (3.3) are ultra-local.

\section{$10.2 \quad$ Ultra-locality}

Properties of spaces $\mathcal{U}_{p}$ of $p$-forms (6.1) with ultra-local $\tau$-kernels are analogous to those with pre-ultra-local ones as we describe now.

Firstly, we observe that

$$
\mathcal{U}_{0}^{0+} * \mathcal{U}_{0}^{0+} \subset \operatorname{Span}\left(\mathcal{U}_{0}^{0+}, \mathcal{I}\right)
$$

Indeed, by definition of pre-ultra-locality, formulae (9.6), (9.7) imply that additional contractions between the $y$-dependent terms will not affect $y$-independent arguments of zeroforms $C$. From relations (9.2)-(9.4) it also follows that the space

$$
\mathcal{U}_{0}:=\operatorname{Span}\left(\mathcal{U}_{0}^{0+}, \mathcal{H}_{0}^{+0}\right)
$$


forms a subspace of $\mathcal{H}_{0}$

$$
\mathcal{U}_{0} * \mathcal{U}_{0} \subset \mathcal{U}_{0} \subset \mathcal{H}_{0}
$$

Using Factorization Lemma (8.9) and formula (8.13) with $q=0$ one has

$$
h_{0,-\infty}\left(\mathcal{U}_{0}\right) \in \mathcal{U}_{0}^{0+} .
$$

Analogously, the ultra-local space of one-forms $\mathcal{U}_{1}^{0+} \subset \mathcal{H}_{1}^{0+}$ and

$$
\mathcal{U}_{1}:=\operatorname{Span}\left(\mathcal{U}_{1}^{0+}, \mathcal{H}_{1}^{+0}\right)
$$

form, respectively, $\mathcal{U}_{0}^{0+}$ — and $\mathcal{U}_{0}$ - bi-modules (modulo elements of $\mathcal{I}$ in the former case)

$$
\begin{array}{rlrl}
\mathcal{U}_{0}^{0+} * \mathcal{U}_{1}^{0+} & \subset \operatorname{Span}\left(\mathcal{U}_{1}^{0+}, \mathcal{I}\right), & \mathcal{U}_{1}^{0+} * \mathcal{U}_{0}^{0+} & \subset \operatorname{Span}\left(\mathcal{U}_{1}^{0+}, \mathcal{I}\right), \\
\mathcal{U}_{0} & * \mathcal{U}_{1} \subset \mathcal{U}_{1}, & \mathcal{U}_{1} * \mathcal{U}_{0} & \subset \mathcal{U}_{1} .
\end{array}
$$

Introducing the space $\widetilde{\mathcal{U}}_{1}^{0+}$ as the ultra-local subspace of $\widetilde{\mathcal{H}}_{1}{ }^{0+}$ and

$$
\widetilde{\mathcal{U}}_{1}:=\operatorname{Span}\left(\widetilde{\mathcal{U}}_{1}{ }^{0+}, \mathcal{H}_{1}^{+0}\right)
$$

and using again product formulae (9.14) and (9.16) we obtain

$$
\begin{array}{rlrl}
\mathcal{U}_{0}^{0+} * \widetilde{\mathcal{U}}_{1}^{0+} & \subset \operatorname{Span}\left(\widetilde{\mathcal{U}}_{1}^{0+}, \mathcal{I}\right) \subset \widetilde{\mathcal{U}}_{1}, & \widetilde{\mathcal{U}}_{1}^{0+} * \mathcal{U}_{0}^{0+} \subset \operatorname{Span}\left(\widetilde{\mathcal{U}}_{1}^{0+}, \mathcal{I}\right) \subset \widetilde{\mathcal{U}}_{1} \\
\mathcal{U}_{0} * \widetilde{\mathcal{U}}_{1} \subset \widetilde{\mathcal{U}}_{1}, & \widetilde{\mathcal{U}}_{1} * \mathcal{U}_{0} \subset \widetilde{\mathcal{U}}_{1}
\end{array}
$$

As above, one can see that by virtue of (9.28), (10.18), (10.19) (10.23) and (10.25) along with Factorization Lemma

$$
\triangle_{0,-\infty}\left(\widetilde{\mathcal{U}}_{1}\right) \subset \mathcal{U}_{0}
$$

and

$$
h_{0,-\infty}\left(\triangle_{0,-\infty}\left(\mathcal{U}_{0} * \widetilde{\mathcal{U}}_{1}\right) * \mathcal{U}_{0}\right) \subset \mathcal{U}_{0}^{0+}, \quad \text { etc. }
$$

\section{Structure relation}

\subsection{Summary}

Let us briefly summarize the key facts of the analysis performed so far.

Limiting contracting homotopy $\triangle_{0,-\infty}$ maps $\mathcal{H}_{2}^{+0}$ to the space $\widetilde{\mathcal{H}}_{1}$ that gives finite preultra-local result under the action of $\triangle_{q,-\infty}$. This implies that the contribution resulting from $\mathcal{I}$ should be kept in the $\theta^{2}$ terms as giving rise to nontrivial $S$ fields in $\widetilde{\mathcal{H}}_{1}$. Also, the parts of $S$ in $\mathcal{I}$ should be kept to compute the contribution to $S * S$ giving rise to higher-order corrections to $S=S_{0}+\tilde{S}$ via

$$
-2 i \mathrm{~d}_{Z} \tilde{S}=-\tilde{S} * \tilde{S}+i(\eta B * \gamma+\bar{\eta} B * \bar{\gamma}) .
$$

For this scheme to work the r.h.s. of the equation on $S$ has to be in $\mathcal{H}_{2}^{+0}$. This is indeed the case in the first order in $C$ since $C * \gamma \in \mathcal{H}_{2}^{+0}$. In this section we show that this property also holds true in the second order thus allowing to apply the limiting homotopy 
formalism to the computation of the second-order in $C$ corrections to the equations on the one-form HS fields $\omega$ leading to a spin-ultra-local result in accordance with PLT and Pre-Ultra-Locality Theorem.

The central result of this section is structure relation that has the form

$$
R_{2}:=\triangle_{a, 0} \triangle_{b, 0}(\gamma) * \gamma-\triangle_{a, 0}(\gamma) * \triangle_{b, 0}(\gamma) \in \mathcal{H}_{2}^{+0}
$$

It plays the key role in the perturbative analysis of the second-order in $C$ contribution to $S$ in the (anti)holomorphic sector. Indeed, the equation on $S_{2}$ in the holomorphic sector has the form

$$
-2 i \mathrm{~d}_{z} S_{2}+S_{1} * S_{1}-i \eta B_{2} * \gamma=0 .
$$

As shown in [9], by virtue of the star-exchange formulae the last two terms turn out to be proportional to $\triangle_{a, 0} \triangle_{b, 0}(\gamma) * \gamma-\triangle_{a, 0}(\gamma) * \triangle_{b, 0}(\gamma)$. By (9.49), (11.2) implies that

$$
S_{2} \in \widetilde{\mathcal{H}}_{1}
$$

As a result, the second-order part of $W_{2}$ generated by $S_{2}$ is not only well defined in the limit $\beta \rightarrow-\infty$ but ultra-local by PLT and Pre-Ultra-Locality Theorem. Note that each of the two terms on the l.h.s. of (11.2) gives divergent contributions to $W_{2}$ in the limit $\beta \rightarrow-\infty$. However the contribution of the whole expression is finite.

\subsection{The proof}

First, from (7.22) it follows that

$$
\triangle_{a, 0}(\gamma)=2\left(z^{\alpha}+a^{\alpha}\right) \theta_{\alpha} \int d \tau \tau \exp \left[i\left(\tau z_{\alpha} y^{\alpha}-(1-\tau) a_{\alpha} y^{\alpha}\right)\right] k
$$

(Recall that at the first order $\triangle_{a, \beta}^{\prime}(\gamma)$ is independent of $\beta$ [1] and $\triangle_{a, 0}^{\prime}(\gamma)=\triangle_{a, 0}(\gamma)$.) Using (6.8) it is straightforward to compute $\triangle_{a, 0}(\gamma) * \triangle_{b, 0}(\gamma)$. The only comment is that the Klein operator $k$ from the first factor of $\gamma$ moved to the right changes a sign of the shift parameter $b$ acting on the fields standing on the left from the expression $\triangle_{a, 0}(\gamma) * \triangle_{b, 0}(\gamma)$. (For more detail see [2].) The final result is

$$
\begin{aligned}
& \triangle_{a, 0}(\gamma) * \triangle_{b, 0}(\gamma)=2 \theta^{\alpha} \theta_{\alpha} \int_{0}^{1} d \tau_{1} \tau_{1} \int_{0}^{1} d \tau_{2} \tau_{2}\left(2 i-\tau_{1} \circ \tau_{2} z_{\alpha} y^{\alpha}\right. \\
& \left.\quad+\left(1-\left(1-\tau_{1}\right)\left(1-\tau_{2}\right)\right)\left(a_{\alpha} b^{\alpha}-\left(a_{\alpha}-b_{\alpha}\right) y^{\alpha}\right)-\left(\tau_{1}\left(1-\tau_{2}\right) b_{\alpha}+\tau_{2}\left(1-\tau_{1}\right) a_{\alpha}\right) z^{\alpha}\right) \\
& \quad \exp i\left[\tau_{1} \circ \tau_{2} z_{\alpha} y^{\alpha}+\left(1-\tau_{1}\right)\left(1-\tau_{2}\right)\left(a_{\alpha} b^{\alpha}-\left(a_{\alpha}-b_{\alpha}\right) y^{\alpha}\right)+\left(\tau_{1}\left(1-\tau_{2}\right) b_{\alpha}+\tau_{2}\left(1-\tau_{1}\right) a_{\alpha}\right) z^{\alpha}\right]
\end{aligned}
$$

where the integral over $s^{\alpha}$ and $t^{\alpha}$ in (6.8) has been evaluated by virtue of

$$
\begin{aligned}
\frac{1}{4 \pi^{2}} \int d^{2} s d^{2} t \exp i s_{\alpha} t^{\alpha} & =1, \quad \frac{1}{4 \pi^{2}} \int d^{2} s d^{2} t s_{\alpha} t^{\alpha} \exp i s_{\alpha} t^{\alpha}=2 i, \\
\int d^{2} s d^{2} t s_{\alpha} \exp i s_{\alpha} t^{\alpha} & =\int d^{2} s d^{2} t t_{\alpha} \exp i s_{\alpha} t^{\alpha}=0 .
\end{aligned}
$$

Now we single out the terms that belong to $\mathcal{I}$. Namely, all terms containing a factor of $\left(1-\tau_{1}\right)\left(1-\tau_{2}\right)$ are of this type because, multiplied by $\tau_{1} \tau_{2}$ from the measure, by $(6.13)$ 
these are dominated by $\left(\tau_{1} \circ \tau_{2}\left(1-\tau_{1} \circ \tau_{2}\right)\right)^{2}$, thus bringing additional degrees both in $\tau_{1} \circ \tau_{2}$ and in $\left(1-\tau_{1} \circ \tau_{2}\right)$. As a result, $\triangle_{a, 0}(\gamma) * \Delta_{b, 0}(\gamma)$ can be represented in the form

$$
\triangle_{a, 0}(\gamma) * \triangle_{b, 0}(\gamma)=X_{1}^{\mathcal{I}}+X_{2}^{\mathcal{I}}+X
$$

where

$$
\begin{aligned}
X_{1}^{\mathcal{I}}= & -2 \theta^{\alpha} \theta_{\alpha} \int_{0}^{1} d \tau_{1} \tau_{1}\left(1-\tau_{1}\right) \int_{0}^{1} d \tau_{2} \tau_{2}\left(1-\tau_{2}\right)\left(a_{\alpha} b^{\alpha}-\left(a_{\alpha}-b_{\alpha}\right) y^{\alpha}\right) \\
& \exp i\left[\tau_{1} \circ \tau_{2} z_{\alpha} y^{\alpha}+\left(1-\tau_{1}\right)\left(1-\tau_{2}\right)\left(a_{\alpha} b^{\alpha}-\left(a_{\alpha}-b_{\alpha}\right) y^{\alpha}\right)+\left(\tau_{1}\left(1-\tau_{2}\right) b_{\alpha}+\tau_{2}\left(1-\tau_{1}\right) a_{\alpha}\right) z^{\alpha}\right]
\end{aligned}
$$

and, using that $\int d \sigma \frac{\partial}{\partial \sigma} f(\sigma x)=f(x)-f(0)$,

$$
\begin{aligned}
X_{2}^{\mathcal{I}}= & 2 \theta^{\alpha} \theta_{\alpha} \int_{0}^{1} d \tau_{1} \tau_{1}\left(1-\tau_{1}\right) \int_{0}^{1} d \tau_{2} \tau_{2}\left(1-\tau_{2}\right) \int_{0}^{1} d \sigma\left(a_{\alpha} b^{\alpha}-\left(a_{\alpha}-b_{\alpha}\right) y^{\alpha}\right) \\
& \left(2 i-\tau_{1} \circ \tau_{2} z_{\alpha} y^{\alpha}+a_{\alpha} b^{\alpha}-\left(a_{\alpha}-b_{\alpha}\right) y^{\alpha}-\left(\tau_{1}\left(1-\tau_{2}\right) b_{\alpha}+\tau_{2}\left(1-\tau_{1}\right) a_{\alpha}\right) z^{\alpha}\right) \\
& \quad \exp i\left[\tau_{1} \circ \tau_{2} z_{\alpha} y^{\alpha}+\sigma\left(1-\tau_{1}\right)\left(1-\tau_{2}\right)\left(a_{\alpha} b^{\alpha}-\left(a_{\alpha}-b_{\alpha}\right) y^{\alpha}\right)+\left(\tau_{1}\left(1-\tau_{2}\right) b_{\alpha}+\tau_{2}\left(1-\tau_{1}\right) a_{\alpha}\right) z^{\alpha}\right] \\
\text { belong to } \mathcal{I}, \text { while } & \\
X= & 2 \theta^{\alpha} \theta_{\alpha} \int_{0}^{1} d \tau_{1} \tau_{1} \int_{0}^{1} d \tau_{2} \tau_{2} \exp i\left(\tau_{1} \circ \tau_{2} z_{\alpha} y^{\alpha}+\left(\tau_{1}\left(1-\tau_{2}\right) b_{\alpha}+\tau_{2}\left(1-\tau_{1}\right) a_{\alpha}\right) z^{\alpha}\right) \\
& \left(2 i-\tau_{1} \circ \tau_{2} z_{\alpha} y^{\alpha}+a_{\alpha} b^{\alpha}-\left(a_{\alpha}-b_{\alpha}\right) y^{\alpha}-\left(\tau_{1}\left(1-\tau_{2}\right) b_{\alpha}+\tau_{2}\left(1-\tau_{1}\right) a_{\alpha}\right) z^{\alpha}\right) .
\end{aligned}
$$

Now we observe that

$$
\begin{aligned}
& \left(\left(1-\tau_{1}\right) \frac{\partial}{\partial \tau_{1}}+\left(1-\tau_{2}\right) \frac{\partial}{\partial \tau_{2}}\right) \tau_{1}\left(1-\tau_{2}\right)=\left(1-\tau_{1}\right)\left(1-\tau_{2}\right)-\tau_{1}\left(1-\tau_{2}\right), \\
& \left(\left(1-\tau_{1}\right) \frac{\partial}{\partial \tau_{1}}+\left(1-\tau_{2}\right) \frac{\partial}{\partial \tau_{2}}\right) \tau_{2}\left(1-\tau_{1}\right)=\left(1-\tau_{1}\right)\left(1-\tau_{2}\right)-\tau_{2}\left(1-\tau_{1}\right)
\end{aligned}
$$

and, hence,

$$
\left(\left(1-\tau_{1}\right) \frac{\partial}{\partial \tau_{1}}+\left(1-\tau_{2}\right) \frac{\partial}{\partial \tau_{2}}\right) \tau_{1} \circ \tau_{2}=2\left(1-\tau_{1}\right)\left(1-\tau_{2}\right)-\tau_{1} \circ \tau_{2} .
$$

This implies,

$$
\begin{aligned}
& i\left(\left(1-\tau_{1}\right) \frac{\partial}{\partial \tau_{1}}+\left(1-\tau_{2}\right) \frac{\partial}{\partial \tau_{2}}\right) \exp i\left(\tau_{1} \circ \tau_{2} z_{\alpha} y^{\alpha}+\left(\tau_{1}\left(1-\tau_{2}\right) b_{\alpha}+\tau_{2}\left(1-\tau_{1}\right) a_{\alpha}\right) z^{\alpha}\right) \\
& =\left(\tau_{1} \circ \tau_{2} z_{\alpha} y^{\alpha}+\left(\tau_{1}\left(1-\tau_{2}\right) b_{\alpha}+\tau_{2}\left(1-\tau_{1}\right) a_{\alpha}\right) z^{\alpha}-\left(1-\tau_{1}\right)\left(1-\tau_{2}\right)\left(2 z_{\alpha} y^{\alpha}+\left(a_{\alpha}+b_{\alpha}\right) z^{\alpha}\right)\right) \\
& \quad \exp i\left(\tau_{1} \circ \tau_{2} z_{\alpha} y^{\alpha}+\left(\tau_{1}\left(1-\tau_{2}\right) b_{\alpha}+\tau_{2}\left(1-\tau_{1}\right) a_{\alpha}\right) z^{\alpha}\right)
\end{aligned}
$$

and, hence,

$$
\begin{aligned}
X= & 2 \theta^{\alpha} \theta_{\alpha} \int_{0}^{1} d \tau_{1} \tau_{1} \int_{0}^{1} d \tau_{2} \tau_{2} \\
& \left(2 i-i\left(\left(1-\tau_{1}\right) \frac{\partial}{\partial \tau_{1}}+\left(1-\tau_{2}\right) \frac{\partial}{\partial \tau_{2}}\right)\right. \\
& \left.\quad+a_{\alpha} b^{\alpha}-\left(a_{\alpha}-b_{\alpha}\right) y^{\alpha}-\left(1-\tau_{1}\right)\left(1-\tau_{2}\right)\left(2 z_{\alpha} y^{\alpha}+\left(a_{\alpha}+b_{\alpha}\right) z^{\alpha}\right)\right) \\
& \exp i\left(\tau_{1} \circ \tau_{2} z_{\alpha} y^{\alpha}+\left(\tau_{1}\left(1-\tau_{2}\right) b_{\alpha}+\tau_{2}\left(1-\tau_{1}\right) a_{\alpha}\right) z^{\alpha}\right) .
\end{aligned}
$$


Integration by parts gives

$$
X=Y+X_{3}^{\mathcal{I}},
$$

where $X_{3}^{\mathcal{I}}$ belongs to $\mathcal{I}$,

$$
\begin{aligned}
\mathcal{I} \ni X_{3}^{\mathcal{I}}= & 2 \theta^{\alpha} \theta_{\alpha} \int_{0}^{1} d \tau_{1} \int_{0}^{1} d \tau_{2}\left(i \tau_{1} \circ \tau_{2}-\tau_{1} \tau_{2}\left(1-\tau_{1}\right)\left(1-\tau_{2}\right)\left(2 z_{\alpha} y^{\alpha}+\left(a_{\alpha}+b_{\alpha}\right) z^{\alpha}\right)\right) \\
& \exp i\left(\tau_{1} \circ \tau_{2} z_{\alpha} y^{\alpha}+\left(\tau_{1}\left(1-\tau_{2}\right) b_{\alpha}+\tau_{2}\left(1-\tau_{1}\right) a_{\alpha}\right) z^{\alpha}\right)
\end{aligned}
$$

and

$$
\begin{aligned}
Y= & 2 \theta^{\alpha} \theta_{\alpha} \int_{0}^{1} d \tau_{1} \tau_{1} \int_{0}^{1} d \tau_{2} \tau_{2}\left(a_{\alpha} b^{\alpha}-\left(a_{\alpha}-b_{\alpha}\right) y^{\alpha}\right) \\
& \times \exp i\left(\tau_{1} \circ \tau_{2} z_{\alpha} y^{\alpha}+\left(\tau_{1}\left(1-\tau_{2}\right) b_{\alpha}+\tau_{2}\left(1-\tau_{1}\right) a_{\alpha}\right) z^{\alpha}\right)
\end{aligned}
$$

is the remaining term in $\triangle_{a, 0}(\gamma) * \triangle_{b, 0}(\gamma)$ that does not belong to $\mathcal{I}$. (Note that the analysis of the holomorphic vertex in [1] contained partial integration being a zero-form image of this one.)

This can now be compared with the expression for $\triangle_{a, 0} \triangle_{b, 0}(\gamma) * \gamma$ obtained in [9] (recall that $\triangle_{a, 0}$ of this paper coincides with $\triangle_{a}$ of [9])

$$
\begin{aligned}
\triangle_{a, 0} \triangle_{b, 0}(\gamma) * \gamma= & 2 \theta^{\alpha} \theta_{\alpha} \int d \sigma_{1} d \sigma_{2} \theta\left(\sigma_{1}\right) \theta\left(\sigma_{2}\right) \theta\left(1-\sigma_{1}-\sigma_{2}\right)\left(a_{\alpha}-b_{\alpha}\right)\left(a^{\alpha}-y^{\alpha}\right) \\
& \times \exp i\left(\left(\sigma_{1}+\sigma_{2}\right) z_{\alpha} y^{\alpha}+\left(\sigma_{1} a_{\alpha}+\sigma_{2} b_{\alpha}\right) z^{\alpha}\right),
\end{aligned}
$$

that is not difficult to obtain directly. We observe that $Y$ and $\Delta_{a} \Delta_{b}(\gamma) * \gamma$ have similar form up to the substitution

$$
\sigma_{1} \rightarrow \tau_{2}\left(1-\tau_{1}\right), \quad \sigma_{2} \rightarrow \tau_{1}\left(1-\tau_{2}\right)
$$

As we show now, this implies (11.2).

Indeed, from (9.17) we see that $S_{1} * S_{1}$ and hence $Y$ belong to $\mathcal{H}_{2}^{0+}$. Due to the factor of $\tau_{1} \tau_{2}$ in the measure of $Y$ (11.20) the dominating part of $Y=Y^{0+}$ comes from $\tau_{1} \sim 1-\varepsilon_{1}$, $\tau_{2} \sim 1-\varepsilon_{2}$ with small $\varepsilon_{1,2}$,

$$
Y \simeq 2 \theta^{\alpha} \theta_{\alpha} \int_{0}^{\epsilon} d \varepsilon_{1} \int_{0}^{\epsilon} d \varepsilon_{2}\left(a_{\alpha} b^{\alpha}-\left(a_{\alpha}-b_{\alpha}\right) y^{\alpha}\right) \exp i\left(\left(\varepsilon_{1}+\varepsilon_{2}\right) z_{\alpha} y^{\alpha}+\left(\varepsilon_{2} b_{\alpha}+\varepsilon_{1} a_{\alpha}\right) z^{\alpha}\right),
$$

where $\epsilon$ is some small parameter. This expression coincides with the part of (11.21) resulting from the integration around small $\sigma_{1}$ and $\sigma_{2}$ that proves (11.2). The precise form of the l.h.s. of (11.2), which is quite tricky, will be presented elsewhere.

Let us stress that property (11.2) has been proven for any parameters $a_{\alpha}$ and $b_{\beta}$. Further simplifications, occur in accordance with Pre-Ultra-Locality Theorem (10.11) eliminating the $y$-dependence from zero-forms $C$ upon application of the limiting contracting homotopy and, if these parameters respect PLT, by Ultra-Locality Theorem implying that the resulting contributions to the sectors of one- and zero-forms in $\theta$ are ultra-local. 


\section{Example: ultra-locality of holomorphic $\Upsilon_{2}(\omega, \omega, C, C)$}

Perturbative analysis sketched in section 5 implies that the quadratic correction to the one-form sector of the field equations is (see also [1])

$$
\Upsilon_{2}(\omega, \omega, C, C)=-h_{0,-\infty}\left(\mathrm{d}_{x} W_{1}+\mathrm{d}_{x} W_{2}+W_{1} * W_{1}+\left\{\omega, W_{2}\right\}_{*}\right),
$$

where

$$
\begin{aligned}
W_{2} & =\frac{1}{2 i} \triangle_{0,-\infty}\left(\mathrm{d}_{x} S_{1}+\mathrm{d}_{x} S_{2}+\left\{W_{1}, S_{1}\right\}_{*}+\left\{\omega, S_{2}\right\}_{*}\right), \\
S_{2} & =\frac{i}{2} \triangle_{0,-\infty}\left(i \eta B_{2} * \gamma-S_{1} * S_{1}\right) .
\end{aligned}
$$

By PLT the holomorphic part of $\Upsilon_{2}(\omega, \omega, C, C)$ belongs to the PLT-even class. Following [1], we consider the PLT-even contracting homotopy $\Delta_{0,-\infty}$ and the respective cohomology projector $h_{0,-\infty}$ allowing to discard the terms containing space-time differential $\mathrm{d}_{x}$. In agreement with [1], the remaining terms will now be shown to be ultra-local by the following computation-independent arguments.

I. The contribution of $W_{1} * W_{1}$ is ultra-local.

Indeed, from [1] one has

$$
\begin{aligned}
W_{1}= & \frac{i \eta}{2} \int \mathrm{d}_{\Delta}^{3} \tau\left\{C\left(y_{1}\right) \bar{*} \omega\left(w_{1}\right) t^{\alpha} z_{\alpha} \exp i\left(\tau_{1} z_{\alpha}\left(y^{\alpha}+p^{\alpha}\right)+t^{\alpha}\left(\tau_{1} z_{\alpha}+\tau_{3} y_{\alpha}-\left(1-\tau_{3}\right) p_{\alpha}\right)\right)\right. \\
& \left.+\omega\left(w_{1}\right) \bar{*} C\left(y_{1}\right) t^{\alpha} z_{\alpha} \exp i\left(\tau_{1} z_{\alpha}\left(y^{\alpha}+p^{\alpha}\right)+t^{\alpha}\left(\tau_{1} z_{\alpha}-\tau_{3} y_{\alpha}+\left(1-\tau_{3}\right) p_{\alpha}\right)\right)\right\}\left.k\right|_{y_{1}=w_{1}=0} \\
& + \text { h.c. }
\end{aligned}
$$

with $t=-i \frac{\partial}{\partial w_{1}}, p=-i \frac{\partial}{\partial y_{1}}$ and convention that h.c. (Hermitean conjugation) swaps barred and unbarred variables along with dotted and undotted indices.

By definition (10.17)

$$
W_{1} \in \mathcal{U}_{0}
$$

Hence by virtue of (10.18) and (10.19) $W_{1} * W_{1} \in \mathcal{U}_{0}$ and $h_{0,-\infty}\left(W_{1} * W_{1}\right) \in \mathcal{U}_{0}$. This means that the contribution of $W_{1} * W_{1}$ to the field equations is ultra-local.

II. The contribution of $\left\{W_{1}, S_{1}\right\}_{*}$ is ultra-local.

Indeed, from [9] one has

$$
S_{1}=\left.\eta \theta^{\alpha} z_{\alpha} \int_{0}^{1} \mathrm{~d} \tau \tau \exp \left(i \tau z_{\alpha}\left(y^{\alpha}+p_{1}^{\alpha}\right)\right) k C\left(y_{1}\right)\right|_{y_{1}=0}+\text { h.c. } .
$$

Hence, by definition (10.23), $S_{1} \in \widetilde{\mathcal{U}}_{1}$ and by virtue of (10.25) along with (12.5) $\left\{W_{1}, S_{1}\right\}_{*} \in \widetilde{\mathcal{U}}_{1} . \quad$ By virtue of $(10.26) \Delta_{0,-\infty}\left(\left\{W_{1}, S_{1}\right\}_{*}\right) \in \mathcal{U}_{0}$. Then (10.18) and (10.19) give

$$
h_{0,-\infty}\left(\left\{\triangle_{0,-\infty}\left(\left\{S_{1}, W_{1}\right\}_{*}\right), \omega\right\}_{*}\right) \in \mathcal{U}_{0} .
$$

Thus, the contribution to the field equations of the PLT-even expression $\left\{W_{1}, S_{1}\right\}_{*}$ is ultra-local. 
III. The contribution of $\left\{\omega, S_{2}\right\}_{*}$ is ultra-local.

Indeed, by virtue of [9] $S_{2}$ (12.3) has a form

$$
S_{2}=-\frac{\eta}{2} \triangle_{0,-\infty}\left(C * C *\left(\triangle_{a, 0} \triangle_{b, 0}(\gamma) * \gamma-\triangle_{a, 0}(\gamma) * \triangle_{b, 0}(\gamma)\right)\right)
$$

where $a_{\alpha}=p_{1 \alpha}+2 p_{2 \alpha}, b_{\alpha}=p_{2 \alpha}$ with $p_{j \alpha}(3.20)$.

Straightforwardly one can make sure that for any zero-form $f_{0}(y)$

$$
f_{0}(y) * f_{2}^{+0} \subset \mathcal{H}_{2}^{+0}, \quad f_{2}^{+0} * f_{0}(y) \subset \mathcal{H}_{2}^{+0} .
$$

Thus by virtue of structure relation (11.2) and Pre-Ultra-Locality Theorem (10.11) it follows that

$$
S_{2} \in \triangle_{0,-\infty}^{\prime} \mathcal{H}_{2}^{+0} \in \widetilde{\mathcal{P}}_{1}
$$

In [2] it was shown in particular that $S_{2}$ is PLT-even. By virtue of (7.37) from (12.10) it follows

$$
\triangle_{0,-\infty}^{\prime} \mathcal{H}_{2}^{+0} \subset \widetilde{\mathcal{U}}_{1}
$$

Since $\omega \in \mathcal{U}_{0},(10.18),(10.22),(10.26)$ and (10.27) give

$$
h_{0,-\infty}\left(\left\{\triangle_{0,-\infty}\left(\left\{S_{2}, \omega\right\}_{*}\right), \omega\right\}_{*}\right) \in \mathcal{U}_{0},
$$

whence the vertex is ultra-local.

\section{Conclusion}

In this paper we have analysed spin-locality of the $4 d$ HS theory in terms of classes of star-product functions that appear in the perturbative analysis of nonlinear equations of [4] based on the $\beta \rightarrow-\infty$ limiting homotopy introduced in [1]. The space $\mathcal{H}$ of starproduct functions that appear in the perturbative analysis was introduced in [10]. It consists of two subspaces $\mathcal{H}=\operatorname{Span}\left(\mathcal{H}^{0+}, \mathcal{H}^{+0}\right)$ such that elements of the zero-form sector in spinor differentials $\mathcal{H}_{0}^{+0} \subset \mathcal{H}^{+0}$ do not contribute to the dynamical equations in the limiting homotopy formalism. This fact is referred to as Factorization Lemma in this paper. Elements of $\mathcal{H}_{0}^{+0}$ give rise to nonlocal contributions to vertices in HS field equations at finite $\beta$, that fits the interpretation of $\mathcal{H}^{0+}$ as a local subalgebra of $\mathcal{H}$ suggested in [10]. Also, we identified the two-sided ideal $\mathcal{I}=\mathcal{H}^{0+} \cap \mathcal{H}^{+0}$ elements of which can be discarded within the limiting homotopy procedure in all sectors of HS field equations that contain HS gauge fields $\omega$.

A priori, application of the limiting homotopy prescription to general elements of $\mathcal{H}$ may not be well defined leading to the HS gauge fields $W$ divergent in the $\beta \rightarrow-\infty$ limit. This does not imply any divergency in the HS equations, that are well defined for any finite $\beta<1$, but rather that inapplicability of the limiting homotopy may indicate that the theory is essentially nonlocal. Hence, it is important to have a sufficient criterion guaranteeing that this does not happen. This is provided by the $\mathcal{H}_{2}^{+0}$ Homotopy Lemma proven in the paper, which states that the limit $\beta \rightarrow-\infty$ is well defined provided that the 
two-form in spinorial differential $\theta$ on the r.h.s. of HS equations on $S$ belongs to $\mathcal{H}^{+0}$. It is shown that this is indeed true in the first and second orders in the zero-forms $C$. In the first order this fact is trivial while in the second it follows from the remarkable Structure Relation proven in section 11.

Another important issue is to have a sufficient criterion for spin-locality of the resulting vertices. This is also found in this paper in the form of Pre-Ultra-Locality Theorem following from the Pfaffian Locality Theorem of [2] and its extension to $\beta$-dependent contracting homotopies given in this paper. Using remarkable form of the limiting contracting homotopy, it is shown that if the conditions of the $\mathcal{H}_{2}^{+0}$ Homotopy Lemma are fulfilled along with PLT conditions, the resulting HS vertex is ultra-local in terminology of [9], i.e. in addition to being spin-local, arguments of the zero-forms $C$ are independent of the spinor variable $y$. Using general properties of the limiting homotopy formalism it is shown that the resulting $\omega^{2} C^{2}$ vertices proportional to $\eta^{2}$ or $\bar{\eta}^{2}$, where $\eta$ is a free complex parameter in the HS theory, must be ultra-local. This is of course in agreement with the detailed analysis of [1]. The developed technique is, however, promising from the perspective of the analysis of higher-order corrections.

It should be stressed that the analysis of this paper is heavily based on the specific form of HS equations (4.1), (4.2) and star product (4.9). In particular, it follows that the only version of the HS theory that admits spin-locality is that with linear function $F_{*}(B)=\eta B$. Possible nonlinear terms in $F_{*}(B)$ contain unremovable spin-non-local terms resulting from star products of the factors of zero-forms $C(Y)$. This explains the distinguished role of the linear function $F_{*}(B)=\eta B$ in HS theory from the holographic perspective: the HS theories with nonlinear $F_{*}(B)$ have some essentially nonlocal boundary duals. (It would be interesting to see which ones, however.)

The approach of this paper, which is applicable not only to the $4 d$ HS theory of [4] but also to HS theory in $3 d$ of [5], any $d$ of [15] and Coxeter HS theories of [16], provides a step towards complete analysis of the level and role of non-locality in HS gauge theory. (For instance, in the model of [15] spin-locality, demanding at most a finite number of contractions between different zero-forms, should take place with respect to Lorentz-covariant components $Y_{A}^{i} V^{A}$ of the auxiliary variables $Y_{A}^{i}$ where $i=1,2$ is the $s p(2)$ vector index, $A=0, \ldots d$ carries the vector representation of $o(d-1,2)$ and $V^{A}$ is the compensator field of the model.) So far it agrees with the conjecture of [2] that HS theory should be spin-local in all orders of the perturbation theory. The identification of the spin-local formulation of the HS gauge theory should make it possible to analyze such important issues as causality and, in the framework of Coxeter HS theory of [16], relation with analogous aspects of String Theory.

The concept of spin-locality underlying analysis of HS interactions in terms of spinors, allows a clear interpretation in terms of usual $x$-space formulation. Namely, as explained in section 3, spin-local theories are space-time local in terms of the original set of fields $\mathcal{C}$ extended by their non-linear local currents $J^{n}\left(\mathcal{C}_{1}, \ldots, \mathcal{C}_{n}\right)$. In other words, in spin-local theories corrections to space-time dynamical equations have a form of local operators in terms of $J^{n}$ with various $n$. The class of spin-local theories sharing this property is just in between local theories with local vertices expressed directly in terms of $\mathcal{C}$ and non-local 
ones where the current corrections themselves can be nonlocal. Note that the difference between local and spin-local theories matters only for the theories with infinite sets of fields as is the case in HS theories. We conjecture that the concept of spin-local theories of infinite sets of fields is just a proper substitute for that of local theories describing finite collections of fields.

The same time we believe that results of [1] and of this paper provide a proper basis for the extension of the study of HS interactions to all higher orders and, in particular, to the $C^{3}$ vertex in the equations for zero-forms that includes the scalar self-interaction vertex. This will make it possible to compare the output of the limiting homotopy prescription in the bulk with the conclusions of the papers $[42,43]$ obtained via holographic reconstruction as well as with the paper [29] based on the light-cone formalism.

An interesting feature of the developed formalism is that it treats differently HS oneforms $\omega$ and zero-forms $C$. In the sector of higher spins this is just what is needed given that zero-forms $C$ contain infinite tails of higher derivatives of Fronsdal fields while oneforms $\omega$ contain at most a finite number of derivatives. However, the general version of the $4 d$ HS theory [4] contains also an infinite set of topological (Killing-like) fields, each carrying at most a finite number of degrees of freedom. In this case the roles of one-forms and zero-forms are just swapped: zero-forms $C^{\text {top }}$ contain finite numbers of derivatives of the topological fields while one-forms $\omega^{\text {top }}$ contain infinite towers of derivatives. This can affect the analysis of locality in the cases when the HS and topological sectors get interacting, that can happen if some of the topological fields acquire a nontrivial VEV. In particular this happens in the $3 d$ HS theory of [5] where the topological sector is related to the dynamical one. From this perspective the results of [1] and of this paper demand further investigation accounting for this phenomenon.

\section{Acknowledgments}

We would like to thank Slava Didenko, Tolya Korybut and Nikita Misuna for fruitful discussions and Alexey Sharapov for a useful comment. We acknowledge a partial support from the Russian Basic Research Foundation Grant No 17-02-00546 and Australian Research Council, project No.DP160103633. The work of OG is partially supported by the FGU FNC SRISA RAS (theme 0065-2019-000736.20.).

\section{A Useful formulae}

As shown in [1], different contracting homotopy operators anticommute

$$
\triangle_{q_{I}, \beta_{I}} \triangle_{q_{J}, \beta_{J}}=-\triangle_{q_{J}, \beta_{J}} \triangle_{q_{I}, \beta_{I}} .
$$

In particular, each of them squares to zero

$$
\triangle_{q, \beta_{I}} \triangle_{q, \beta_{I}}=0 .
$$

Also,

$$
h_{q, \beta_{I}} \triangle_{q, \beta_{I}}=0, \quad \triangle_{q_{I}, \beta_{I}} h_{q_{J}, \beta_{J}}=0, \quad h_{q_{I}, \beta_{I}} h_{q_{J}, \beta_{J}}=h_{q_{J}, \beta_{J}} .
$$


Redefined contracting homotopy operators

$$
\triangle_{q, \beta}^{\prime}:=\triangle_{(1-\beta) q, \beta}, \quad h_{q, \beta}^{\prime}:=h_{(1-\beta) q, \beta}
$$

obey star-exchange formulae of [9]

$$
\begin{aligned}
& \triangle_{q, \beta}^{\prime}(a(y) * f(z, y, k, \theta))=a(y) * \triangle_{q+q_{a}, \beta}^{\prime}(f(z, y, k, \theta)), \\
& \triangle_{q, \beta}^{\prime}(f(z, y, k, \theta) * a(y))=\triangle_{q-q_{a}, \beta}^{\prime}(f(z, y, k, \theta)) * a(y)
\end{aligned}
$$

and

$$
\begin{aligned}
& h_{q, \beta}^{\prime}(a(y) * f(z, y, k, \theta))=a(y) * h_{q+q_{a}, \beta}^{\prime}(f(z, y, k, \theta)), \\
& h_{q, \beta}^{\prime}(f(z, y, k, \theta) * a(y))=h_{q-q_{a}, \beta}^{\prime}(f(z, y, k, \theta)) * a(y),
\end{aligned}
$$

where $q_{a}$ represents the shift of the argument of $a(y)$.

Using that $\gamma * a(y)=a(y) * \gamma$ we obtain following [9]

$$
\triangle_{q, \beta}^{\prime}(\gamma) * a(y)=a(y) * \triangle_{q+2 q_{a}, \beta}^{\prime}(\gamma) .
$$

\section{B Contracting homotopy derivation}

Here we outline the main steps of the derivation of formula (7.27) following [1] where it was derived for the case of $q=0$. Applying (7.21) to (6.1) we obtain

$$
\begin{aligned}
\triangle_{q, \beta} f(z, y, \theta)= & \frac{1}{(2 \pi)^{2}} \int d^{2} u d^{2} v \int_{0}^{1} d \tau \int_{0}^{1} d t t^{p-1} \exp i\left[v_{\beta} u^{\beta}+\tau(t z+(1-t)(u-q))_{\alpha}(\beta v+y)^{\alpha}\right] \\
& \times(z+q-u)^{\alpha} \frac{\partial}{\partial \theta^{\alpha}} \phi(\tau(t z+(1-t)(u-q)),(1-\tau)(\beta v+y), \tau \theta, \tau) .
\end{aligned}
$$

Now, shifting $u \rightarrow u+q$ and introducing new integration variables,

$$
\tau_{1}=t \tau, \quad \tau=\tau_{1}+\tau_{2}, \quad 1-\tau=\tau_{3},
$$

with the Jacobian

$$
\operatorname{det}\left|\frac{\partial \tau, t}{\partial \tau_{i}}\right|=\left(\tau_{1}+\tau_{2}\right)^{-1}
$$

we obtain

$$
\begin{aligned}
\triangle_{q, \beta} f(z, y, \theta)= & \int \frac{d^{2} u d^{2} v}{(2 \pi)^{2}} \int d_{+}^{3} \tau \frac{\tau_{1}^{p-1}}{\left(\tau_{1}+\tau_{2}\right)^{p}} \\
& \times \delta\left(1-\sum_{i=1}^{3} \tau_{i}\right) \exp i\left[v_{\beta}\left(u^{\beta}+q^{\beta}\right)+\left(\tau_{1} z+\tau_{2} u\right)_{\beta}(\beta v+y)^{\beta}\right] \\
& \left.(z-u)^{\alpha} \frac{\partial}{\partial \theta^{\alpha}} \phi\left(\tau_{1} z+\tau_{2} u\right), \tau_{3}(\beta v+y),\left(\tau_{1}+\tau_{2}\right) \theta, \tau_{1}+\tau_{2}\right) .
\end{aligned}
$$

Then, shifting the integration variables

$$
u_{\alpha} \rightarrow u_{\alpha}+\frac{\tau_{1} \beta}{1-\tau_{2} \beta} z_{\alpha}, \quad v_{\alpha} \rightarrow\left(1-\tau_{2} \beta\right)^{-1}\left(v_{\alpha}+\tau_{2} y_{\alpha}\right),
$$


we have

$$
\begin{aligned}
\triangle_{q, \beta} f(z, y, \theta)= & \int \frac{d^{2} u d^{2} v}{(2 \pi)^{2}} \int d_{+}^{3} \tau \delta\left(1-\sum_{i=1}^{3} \tau_{i}\right)\left(\tau_{1}\right)^{p-1}\left(1-\beta \tau_{2}\right)^{-3} \\
& \exp i\left[v_{\beta} u^{\beta}+\frac{\tau_{1}}{\left(1-\beta \tau_{2}\right)} z_{\alpha} y^{\alpha}+\frac{1}{1-\tau_{2} \beta}\left(v_{\alpha}+\tau_{2} y_{\alpha}\right) q^{\alpha}\right] \\
& \left(\left(1-\left(\tau_{1}+\tau_{2}\right) \beta\right) z-\left(1-\beta \tau_{2}\right) u\right)^{\alpha} \\
& \frac{\partial}{\partial \theta^{\alpha}} \phi\left(\frac{\tau_{1}}{\left(1-\beta \tau_{2}\right)} z+\tau_{2} u, \frac{\tau_{3}}{1-\beta \tau_{2}}(y+\beta v), \theta, \tau_{1}+\tau_{2}\right) .
\end{aligned}
$$

To reduce this expression to the desired form (6.1) we finally change variables to

$$
\tau_{1}^{\prime}=\frac{\tau_{1}}{1-\beta \tau_{2}}, \quad \tau_{3}^{\prime}=\frac{\tau_{3}}{1-\beta \tau_{2}}, \quad \tau_{2}^{\prime}=\frac{(1-\beta) \tau_{2}}{1-\beta \tau_{2}} .
$$

This simplicial map preserves the class of simplices of unit perimeter in the sense that

$$
\sum_{i=1}^{3} \tau_{i}^{\prime}=1
$$

as a consequence of $\sum_{i=1}^{3} \tau_{i}=1$. The Jacobian is

$$
\operatorname{det}\left|\frac{\partial \tau_{i}^{\prime}}{\partial \tau_{j}}\right|=\frac{1-\beta}{\left(1-\beta \tau_{2}\right)^{3}} .
$$

Using also that

$$
1-\beta \tau_{2}=\frac{1-\beta}{1-\beta\left(1-\tau_{2}^{\prime}\right)}
$$

and shifting

$$
u \rightarrow u-\frac{1-\beta\left(1-\tau_{2}^{\prime}\right)}{1-\beta} q,
$$

we finally obtain (7.27) after discarding primes and the substitution $q \rightarrow(1-\beta) q$.

Open Access. This article is distributed under the terms of the Creative Commons Attribution License (CC-BY 4.0), which permits any use, distribution and reproduction in any medium, provided the original author(s) and source are credited.

\section{References}

[1] V.E. Didenko, O.A. Gelfond, A.V. Korybut and M.A. Vasiliev, Limiting Shifted Homotopy in Higher-Spin Theory and Spin-Locality, JHEP 12 (2019) 086 [arXiv:1909.04876] [INSPIRE].

[2] O.A. Gelfond and M.A. Vasiliev, Homotopy Operators and Locality Theorems in Higher-Spin Equations, Phys. Lett. B 786 (2018) 180 [arXiv:1805.11941] [INSPIRE].

[3] M.A. Vasiliev, Consistent equation for interacting gauge fields of all spins in (3+1)-dimensions, Phys. Lett. B 243 (1990) 378 [INSPIRE].

[4] M.A. Vasiliev, More on equations of motion for interacting massless fields of all spins in (3+1)-dimensions, Phys. Lett. B 285 (1992) 225 [INSPIRE]. 
[5] S.F. Prokushkin and M.A. Vasiliev, Higher spin gauge interactions for massive matter fields in 3-D AdS space-time, Nucl. Phys. B 545 (1999) 385 [hep-th/9806236] [INSPIRE].

[6] M.A. Vasiliev, Current Interactions and Holography from the 0-Form Sector of Nonlinear Higher-Spin Equations, JHEP 10 (2017) 111 [arXiv:1605.02662] [INSPIRE].

[7] O.A. Gelfond and M.A. Vasiliev, Current Interactions from the One-Form Sector of Nonlinear Higher-Spin Equations, Nucl. Phys. B 931 (2018) 383 [arXiv:1706.03718] [INSPIRE].

[8] M.A. Vasiliev, On the Local Frame in Nonlinear Higher-Spin Equations, JHEP 01 (2018) 062 [arXiv: 1707.03735] [INSPIRE].

[9] V.E. Didenko, O.A. Gelfond, A.V. Korybut and M.A. Vasiliev, Homotopy Properties and Lower-Order Vertices in Higher-Spin Equations, J. Phys. A 51 (2018) 465202 [arXiv: 1807.00001] [INSPIRE].

[10] M.A. Vasiliev, Star-Product Functions in Higher-Spin Theory and Locality, JHEP 06 (2015) 031 [arXiv: 1502.02271] [inSPIRE].

[11] I.R. Klebanov and A.M. Polyakov, AdS dual of the critical $O(N)$ vector model, Phys. Lett. B 550 (2002) 213 [hep-th/0210114] [INSPIRE].

[12] S. Giombi and X. Yin, Higher Spin Gauge Theory and Holography: The Three-Point Functions, JHEP 09 (2010) 115 [arXiv:0912.3462] [INSPIRE].

[13] O. Aharony, G. Gur-Ari and R. Yacoby, $d=3$ Bosonic Vector Models Coupled to Chern-Simons Gauge Theories, JHEP 03 (2012) 037 [arXiv:1110.4382] [INSPIRE].

[14] S. Giombi, S. Minwalla, S. Prakash, S.P. Trivedi, S.R. Wadia and X. Yin, Chern-Simons Theory with Vector Fermion Matter, Eur. Phys. J. C 72 (2012) 2112 [arXiv:1110.4386] [INSPIRE].

[15] M.A. Vasiliev, Nonlinear equations for symmetric massless higher spin fields in (A)dS(d), Phys. Lett. B 567 (2003) 139 [hep-th/0304049] [INSPIRE].

[16] M.A. Vasiliev, From Coxeter Higher-Spin Theories to Strings and Tensor Models, JHEP 08 (2018) 051 [arXiv: 1804.06520] [INSPIRE].

[17] V.E. Didenko and M.A. Vasiliev, Static BPS black hole in 4 d higher-spin gauge theory, Phys. Lett. B 682 (2009) 305 [Erratum ibid. B 722 (2013) 389] [arXiv:0906.3898] [INSPIRE].

[18] C. Iazeolla and P. Sundell, Families of exact solutions to Vasiliev's $4 D$ equations with spherical, cylindrical and biaxial symmetry, JHEP 12 (2011) 084 [arXiv:1107.1217] [INSPIRE].

[19] C. Iazeolla and P. Sundell, Biaxially symmetric solutions to 4 D higher-spin gravity, J. Phys. A 46 (2013) 214004 [arXiv:1208.4077] [INSPIRE].

[20] C. Fronsdal, Massless Fields with Integer Spin, Phys. Rev. D 18 (1978) 3624 [InSPIRE].

[21] C. Fronsdal, Singletons and Massless, Integral Spin Fields on de Sitter Space (Elementary Particles in a Curved Space. 7., Phys. Rev. D 20 (1979) 848 [InSPIRE].

[22] J. Fang and C. Fronsdal, Massless Fields with Half Integral Spin, Phys. Rev. D 18 (1978) 3630 [INSPIRE].

[23] J. Fang and C. Fronsdal, Massless, Half Integer Spin Fields in de Sitter Space, Phys. Rev. D 22 (1980) 1361 [INSPIRE].

[24] M.A. Vasiliev, Consistent Equations for Interacting Massless Fields of All Spins in the First Order in Curvatures, Annals Phys. 190 (1989) 59 [INSPIRE]. 
[25] A.K.H. Bengtsson, I. Bengtsson and L. Brink, Cubic Interaction Terms for Arbitrary Spin, Nucl. Phys. B 227 (1983) 31 [inSPIRE].

[26] F.A. Berends, G.J.H. Burgers and H. Van Dam, On spin three selfinteractions, Z. Phys. C 24 (1984) 247 [INSPIRE].

[27] F.A. Berends, G.J.H. Burgers and H. van Dam, On the Theoretical Problems in Constructing Interactions Involving Higher Spin Massless Particles, Nucl. Phys. B 260 (1985) 295 [INSPIRE].

[28] E.S. Fradkin and M.A. Vasiliev, On the Gravitational Interaction of Massless Higher Spin Fields, Phys. Lett. B 189 (1987) 89 [InSPIRE].

[29] R.R. Metsaev, Poincaré invariant dynamics of massless higher spins: Fourth order analysis on mass shell, Mod. Phys. Lett. A 6 (1991) 359 [INSPIRE].

[30] R.R. Metsaev, S matrix approach to massless higher spins theory. 2: The case of internal symmetry, Mod. Phys. Lett. A 6 (1991) 2411 [INSPIRE].

[31] O.V. Shaynkman and M.A. Vasiliev, Scalar field in any dimension from the higher spin gauge theory perspective, Theor. Math. Phys. 123 (2000) 683 [hep-th/0003123] [INSPIRE].

[32] X. Bekaert, S. Cnockaert, C. Iazeolla and M.A. Vasiliev, Nonlinear higher spin theories in various dimensions, in Higher spin gauge theories: Proceedings, 1st Solvay Workshop: Brussels, Belgium, 12-14 May, 2004, pp. 132-197, hep-th/0503128 [INSPIRE].

[33] O.A. Gelfond and M.A. Vasiliev, Higher rank conformal fields in the Sp(2M) symmetric generalized space-time, Theor. Math. Phys. 145 (2005) 1400 [hep-th/0304020] [INSPIRE].

[34] O.A. Gelfond and M.A. Vasiliev, Unfolded Equations for Current Interactions of $4 d$ Massless Fields as a Free System in Mixed Dimensions, J. Exp. Theor. Phys. 120 (2015) 484 [arXiv: 1012.3143] [INSPIRE].

[35] O.A. Gelfond and M.A. Vasiliev, Higher-Rank Fields and Currents, JHEP 10 (2016) 067 [arXiv:1312.6673] [INSPIRE].

[36] C. Sleight and M. Taronna, Higher-Spin Gauge Theories and Bulk Locality, Phys. Rev. Lett. 121 (2018) 171604 [arXiv:1704.07859] [INSPIRE].

[37] M.A. Vasiliev, Higher spin gauge theories: Star product and AdS space, hep-th/9910096 [INSPIRE].

[38] O.A. Gelfond and M.A. Vasiliev, Operator algebra of free conformal currents via twistors, Nucl. Phys. B 876 (2013) 871 [arXiv:1301.3123] [inSPIRE].

[39] E. Sezgin and P. Sundell, Holography in $4 D$ (super) higher spin theories and a test via cubic scalar couplings, JHEP 07 (2005) 044 [hep-th/0305040] [INSPIRE].

[40] S. Giombi and X. Yin, The Higher Spin/Vector Model Duality, J. Phys. A 46 (2013) 214003 [arXiv:1208.4036] [INSPIRE].

[41] N. Boulanger, P. Kessel, E.D. Skvortsov and M. Taronna, Higher spin interactions in four-dimensions: Vasiliev versus Fronsdal, J. Phys. A 49 (2016) 095402 [arXiv:1508.04139] [INSPIRE].

[42] X. Bekaert, J. Erdmenger, D. Ponomarev and C. Sleight, Quartic AdS Interactions in Higher-Spin Gravity from Conformal Field Theory, JHEP 11 (2015) 149 [arXiv: 1508.04292] [INSPIRE].

[43] D. Ponomarev, A Note on (Non)-Locality in Holographic Higher Spin Theories, Universe 4 (2018) 2 [arXiv: 1710.00403] [INSPIRE]. 